\title{
LARGE CARDINALS AND STRONG MODEL THEORETIC TRANSFER PROPERTIES
}

BY

MATTHEW FOREMAN

ABSTRACT. In this paper we prove the following theorem: $\operatorname{Con}(\mathrm{ZFC}+$ there is a 2-huge cardinal) $\Rightarrow$ for all $n$

$$
\operatorname{Con}\left(\text { ZFC }+\left(\aleph_{n+3}, \aleph_{n+2}, \aleph_{n+1}\right) \rightarrow\left(\aleph_{n+2}, \aleph_{n+1}, \aleph_{n}\right)\right) .
$$

We do this by using iterated forcing to collapse the 2-huge cardinal to $\aleph_{n+1}$ and extending the elementary embedding generically.

1. Introduction. The theorem in this paper is from a genre of theorems known as downward transfer theorems. Archetypical of these is the downward LöwenheimSkolem theorem. In modern times there have been generalizations of the Löwenheim-Skolem theorem both to languages stronger than first order predicate logic and to languages with a distinguished predicate. One of the first theorems along these lines is due to Vaught. We start with a definition:

Definition. We say $\langle\kappa, \lambda\rangle \rightarrow\left\langle\kappa^{\prime}, \lambda^{\prime}\right\rangle$ iff given a structure $\mathfrak{A}$ in a countable language with a distinguished predicate $U$ such that $|\mathfrak{A}|=\kappa,\left|U^{\mathfrak{A}}\right|=\lambda$, there is a $\mathfrak{B} \equiv \mathfrak{A}$ with $|\mathfrak{B}|=\kappa^{\prime},\left|U^{\mathfrak{B}}\right|=\lambda^{\prime}$.

Theorem (VAught). $\left\langle\boldsymbol{\kappa}^{+}, \kappa\right\rangle \rightarrow\left\langle\boldsymbol{\aleph}_{1}, \boldsymbol{\aleph}_{0}\right\rangle$.

There is a strengthening of this notion.

DEFINITION. $\langle\kappa, \lambda\rangle \rightarrow\left\langle\kappa^{\prime}, \lambda^{\prime}\right\rangle$ iff for every structure $\mathfrak{A}$ in a countable language with a distinguished predicate $U$ such that $|\mathfrak{A}|=\kappa,\left|U^{\mathfrak{A}}\right|=\lambda$, there is an elementary substructure $\mathfrak{B}$ of $\mathfrak{A}$ such that $|\mathfrak{B}|=\kappa^{\prime}$ and $\left|U^{\mathfrak{B}}\right|=\lambda^{\prime}$.

Chang conjectured that $\left(\aleph_{2}, \aleph_{1}\right) \rightarrow\left(\aleph_{1}, \aleph_{0}\right)$. In 1967 , Silver established the consistency of Chang's Conjecture:

Theorem (Silver).

$\operatorname{Con}(\mathrm{ZFC}+$ there is a Ramsey cardinal $) \Rightarrow \operatorname{Con}\left(\mathrm{ZFC}+\left(\aleph_{2}, \aleph_{1}\right) \rightarrow\left(\aleph_{1}, \aleph_{0}\right)\right)$.

After Kunen did his work on $0^{\#}$ it became clear that this could not be proved in ZFC alone:

Proposition. $\left(\aleph_{2}, \aleph_{1}\right) \rightarrow\left(\aleph_{1}, \aleph_{0}\right)$ implies for all reals $x, x^{\#}$ exists.

Received by the editors December 10, 1980.

1980 Mathematics Subject Classification. Primary 03C55, 03E35; Secondary 03E55.

Key words and phrases. Chang's Conjecture, large cardinals, iterated forcing, master conditions. 
Proof. We apply the transfer theorem to $\mathfrak{A}=\left\langle L_{\aleph_{2}}[x] ; \aleph_{1}, \dot{x}, E\right\rangle$. Let $\mathfrak{B} \prec \mathfrak{A}$ of type $\left\langle\boldsymbol{\aleph}_{1}, \boldsymbol{\aleph}_{0}\right\rangle$. Then $\mathfrak{B}$ is canonically isomorphic to some transitive $\left\langle L_{\delta}[x], \alpha, \dot{x}, E\right\rangle$ via a collapsing map $\pi$. Consider $\pi^{-1}:\left\langle L_{\delta}[x] ; \alpha, \dot{x}, E\right\rangle \rightarrow\left\langle L_{\aleph_{2}}[x] ; \aleph_{1}, x, E\right\rangle$. Since $\alpha$ is countable, $\pi^{-1}$ moves some countable ordinal. Let $\beta$ be the last ordinal moved by $\pi^{-1}$. Since $\left|L_{\delta}[x]\right|=\boldsymbol{\aleph}_{1}, \delta \geqslant \boldsymbol{\aleph}_{1}$ and so

$$
\mathscr{P}(\beta)^{L_{\delta}[x]}=\mathscr{P}(\beta)^{L[x]} .
$$

Let $U=\left\{y \subseteq \beta: \beta \in \pi^{-1}(y)\right\}$. Standard arguments show that $U$ is a well-founded $L[x]$-ultra-filter on $\beta$, so by Kunen $x \#$ exists.

Kunen used a construction from a huge cardinal to get an $\boldsymbol{\aleph}_{2}$-saturated ideal on $\boldsymbol{\aleph}_{1}$. He remarked that in his model $\left(\boldsymbol{\aleph}_{2}, \boldsymbol{\aleph}_{1}\right) \rightarrow\left(\boldsymbol{\aleph}_{1}, \boldsymbol{\aleph}_{0}\right)$. Laver used a variation on Kunen's construction to show

THEOREM (LAVER). Con(ZFC + there is a huge cardinal $) \Rightarrow$ for every $n$

$$
\operatorname{Con}\left(\mathrm{ZFC}+\left(\boldsymbol{\aleph}_{n+2}, \boldsymbol{\aleph}_{n+1}\right) \rightarrow\left(\boldsymbol{\aleph}_{n+1}, \boldsymbol{\aleph}_{n}\right)\right) \text {. }
$$

Laver essentially used Kunen's construction with closed conditions which necessitated an additional chain condition argument.

We now give some more definitions:

Let $j: V \rightarrow M$ be an elementary embedding of $V$ into a transitive class $M$. Let $\kappa_{0}$ be the first ordinal moved by $j$. ( $\kappa_{0}$ is called the critical point of $j$.) Let $\kappa_{i+1}=j\left(\kappa_{i}\right)$.

Definition. $j$ is called $n$-huge iff $M$ is closed under $\kappa_{n}$ sequences (i.e., ${ }^{\kappa_{n}} M \subseteq M$ ). $\kappa$ is called an $n$-huge cardinal iff it is the critical point of some $n$-huge embedding. ${ }^{1}$

The main result of this paper is:

THEOREM. Con(ZFC + there is a 2-huge cardinal $) \Rightarrow$ for all $n$,

$$
\operatorname{Con}\left(\mathrm{ZFC}+\left(\boldsymbol{\aleph}_{n+3}, \boldsymbol{\aleph}_{n+2}, \boldsymbol{\aleph}_{n+1}\right) \rightarrow\left(\boldsymbol{\aleph}_{n+2}, \boldsymbol{\aleph}_{n+1}, \boldsymbol{\aleph}_{n}\right)\right) \text {. }
$$

$\left((\kappa, \lambda, \mu) \rightarrow\left(\kappa^{\prime}, \lambda^{\prime}, \mu^{\prime}\right)\right.$ is the obvious generalization of the transfer property for two predicates instead of one.)

REMARK. $\left(\boldsymbol{\aleph}_{n+3}, \boldsymbol{\aleph}_{n+2}, \boldsymbol{\aleph}_{n+1}\right) \rightarrow\left(\boldsymbol{\aleph}_{n+2}, \boldsymbol{\aleph}_{n+1}, \boldsymbol{\aleph}_{n}\right)$ implies

(a) $\left(\boldsymbol{\aleph}_{n+3}, \boldsymbol{\aleph}_{n+1}\right) \rightarrow\left(\boldsymbol{\aleph}_{n+2}, \boldsymbol{\aleph}_{n}\right)$,

(b) $\left(\boldsymbol{\aleph}_{n+3}, \boldsymbol{\aleph}_{n+2}\right) \rightarrow\left(\boldsymbol{\aleph}_{n+1}, \boldsymbol{\aleph}_{n}\right)$.

It is easy to describe the rough strategy of the proof of these results:

Let $j$ be a 2-huge embedding. Suppose $\mathfrak{A}=\left\langle\kappa_{2} ; \kappa_{1}, \kappa_{0}, f_{i}, \mathbf{R}_{k}, c_{e}\right\rangle(i, k, e \in \omega)$ is a fully Skolemized structure of type $\left(\kappa_{2}, \kappa_{1}, \kappa_{0}\right)$, then

$$
j(\mathfrak{U})=\left\langle\kappa_{3} ; \kappa_{2}, \kappa_{1}, j\left(f_{i}\right), j\left(\mathbf{R}_{k}\right), j\left(c_{e}\right)\right\rangle_{i, k, e \in \omega} .
$$

Claim. $j^{\prime \prime} \mathfrak{U} \prec j(\mathfrak{U})$.

Proof. It is enough to show that $j^{\prime \prime} \mathfrak{A}$ is closed under the functions $j\left(f_{i}\right)$. Let $\vec{\beta} \in j^{\prime \prime} \mathfrak{U}^{<\omega}$. Then $\vec{\beta}=j(\vec{\alpha})$ for some $\vec{\alpha} \in \mathfrak{U}^{<\omega}$. By elementarity of $j, j\left(f_{i}\right)(\vec{\beta})=$ $j\left(f_{i}(\vec{\alpha})\right)$, but $j\left(f_{i}(\vec{\alpha})\right) \in j^{\prime \prime} \mathfrak{A}$.

\footnotetext{
${ }^{1}$ For background about huge and other large cardinals we refer the reader to R. M. Solovay, W. N. Reinhardt and A. Kanamori [5].
} 
Let us examine $j^{\prime \prime} \mathfrak{Q}$ :

$\left|j^{\prime \prime} \mathfrak{A}\right|=\kappa_{2}$, since $|\mathfrak{A}|=\kappa_{2}$,

$\left|j^{\prime \prime} \mathfrak{U} \cap \kappa_{2}\right|=\kappa_{1}$, since $j\left(\kappa_{1}\right)=\kappa_{2}$ and $j$ is order-preserving.

$\left|j^{\prime \prime} \mathfrak{Q} \cap \kappa_{1}\right|=\kappa_{0}$, since $j\left(\kappa_{0}\right)=\kappa_{1}$ and $\kappa_{0}$ is the critical point of $j$.

We want to bring this situation down to the $\boldsymbol{\kappa}_{n}$ 's. For example, if we could find a partial order $\mathbf{P}$ such that

(1) there are generic objects $G, \hat{G}$ for $\mathbf{P}$ and $j(\mathbf{P})$ respectively with the property that for all $p \in \mathbf{P}$,

$$
p \in G \text { iff } j(p) \in \hat{G},
$$

and

(2) $\kappa_{0}=\boldsymbol{\aleph}_{1}^{V[G]}, \kappa_{1}=\boldsymbol{\aleph}_{2}^{V[G]}, \kappa_{2}=\boldsymbol{\kappa}_{3}^{V[G]}$,

then we could build a generic elementary embedding $\hat{j}$ such that

$$
\hat{j}: V[G] \rightarrow M[\hat{G}],
$$

with $\hat{j}$ extending $j$.

We can picture this as follows:

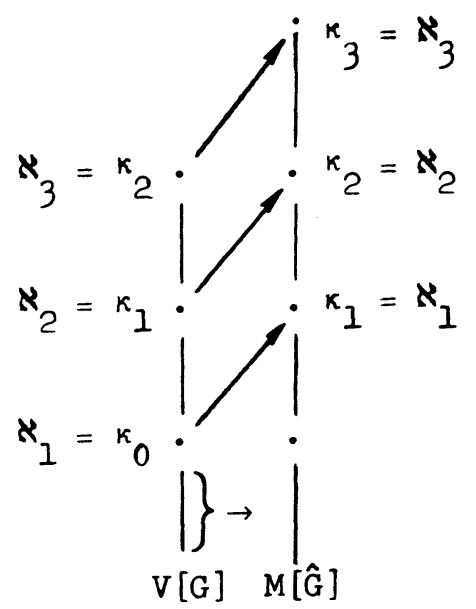

By elementarity:

$$
M[\hat{G}] \vDash \kappa_{1}=\boldsymbol{\aleph}_{1}, \quad \kappa_{2}=\boldsymbol{\kappa}_{2}, \quad \kappa_{3}=\boldsymbol{\kappa}_{3} .
$$

Claim. $V[G] \equiv\left(\boldsymbol{\aleph}_{3}, \boldsymbol{\aleph}_{2}, \boldsymbol{\aleph}_{1}\right) \rightarrow\left(\boldsymbol{\aleph}_{2}, \boldsymbol{\aleph}_{1}, \boldsymbol{\aleph}_{0}\right)$.

Proof. Suppose not. Let $\mathfrak{A}=\left\langle\kappa_{2}, \kappa_{1}, \kappa_{0}, f_{i}, \ldots\right\rangle$ be a fully Skolemized counterexample in $V[G] . M[\hat{G}] \vDash \hat{j}(\mathfrak{A})$ is a counterexample. As before, we can check that $\hat{j}^{\prime \prime} \mathfrak{A}$ is a substructure of $\hat{j}(\mathfrak{A})$, and hence an elementary substructure. Looking at $\hat{j}^{\prime \prime} \mathfrak{A}$ we check

(a) $\left|\hat{j}^{\prime \prime} \mathfrak{Q}\right|=\left|\kappa_{2}\right|^{M[\hat{G}]}=\boldsymbol{\kappa}_{2}$,

(b) $\left|\hat{j}^{\prime \prime} \mathfrak{A} \cap \kappa_{2}\right|=\left|\kappa_{1}\right|^{M[\hat{G}]}=\aleph_{1}$,

(c) $\left|\hat{j}^{\prime \prime} \mathfrak{U} \cap \kappa_{1}\right|=\left|\kappa_{0}\right|^{M[\hat{G}]}=\aleph_{0}$.

Hence in $M[\hat{G}], \hat{j}^{\prime \prime} \mathfrak{A}$ is of type $\left(\aleph_{2}, \aleph_{1}, \aleph_{0}\right)$ and an elementary substructure of $\hat{j}(\mathfrak{A})$, contradicting $\hat{j}(\mathfrak{A})$ being a counterexample. 
If we could extend the elementary embedding of a 3-huge cardinal, we would get a four cardinal theorem. From an $n$-huge we could get an $n+1$ cardinal theorem. Finally, if we had a $j: V \rightarrow M$, with $R(\lambda) \subseteq M$ (where $\lambda=\sup _{i \in \omega} \kappa_{i}$ ) and a partial order $\mathbf{P}$ such that $V^{\mathbf{P}} \vDash \lambda=\aleph_{\omega}$ and if we could generically extend the elementary embedding $\hat{j}: V^{\mathbf{P}} \rightarrow M^{j(\mathbf{P})}$, then, by similar arguments we see that $V^{\mathbf{P}} \vDash \boldsymbol{\aleph}_{\omega}$ is Jonsson.

Getting a $\mathbf{P}$ as desired for a 2-huge embedding is rather difficult. The construction will in some ways resemble the Kunen construction for an $\boldsymbol{\aleph}_{2}$-saturated ideal on $\boldsymbol{\aleph}_{1}$. In that construction he builds a "universal" partial order to collapse $\kappa_{0}$ to $\aleph_{1}$ and follows it by the Silver collapse of $\kappa_{1}$ to $\aleph_{2}$. Using universality on the " $j$-side" he can construct a master condition for the Silver forcing and thus extend the elementary embedding. In imitating the Kunen construction we run into a conflict between universality and master conditions. This will be discussed further in $\S 4$.

$\$ 2$ will review basic forcing definitions and establish some conventions that will be followed in the paper. In $\S 3$, we will review parts of Kunen's construction and present a lemma involving a commutative diagram. \$4 will be an attempt at motivating the rest of the argument. It will try to explicate some of the difficulties encountered in generalizing Kunen's argument and the way that they have been remedied. $\S 5$ is an easy section laying the groundwork for the techniques used in $\$ \S 6$ and 7. In $\$ 6$ we define the partial orders and the maps between them that will make diagrams like the one in $\$ 3$ commute. $\$ 7$ shows that the diagrams actually do commute and that this gives us a master condition. In $\$ 8$ we finish the argument and clear up a few details.

We will use the following notation throughout: $|X|$ will stand for the cardinality of $X$. We will use the abbreviation "o.t P" to stand for the order type of a partial order $\mathbf{P}$.

We will assume that the reader is familiar with iterated forcing. The argument as presented here is self-contained, but is motivated by the Kunen construction in [2].

2. In this section we will review a few basic definitions and lemmas about forcing and introduce a slight variation on the normal two-stage iteration.

Definition 2.1. Let $\mathbf{P}$ and $\mathbf{Q}$ be partial orders and $e: \mathbf{P} \rightarrow \mathbf{Q}$ be a one-to-one function such that

(1) $p_{1} \leqslant_{\mathbf{P}} p_{2}$ implies $e\left(p_{1}\right) \leqslant_{\mathbf{Q}} e\left(p_{2}\right)$,

(2) If $A \subseteq \mathbf{P}$ is a maximal antichain in $\mathbf{P}$ then $e^{\prime \prime} A \subseteq \mathbf{Q}$ is a maximal antichain in Q.

Then we call $e$ a neat embedding and we write $e: \mathbf{P} \hookrightarrow \mathbf{Q}$. (We will use this notation to imply that $e$ is a neat embedding.)

We will assume throughout that all our partial orders are separative and have a greatest element 1 . Given two conditions, $p$ and $q, p \leqslant q$ means $p$ is stronger (i.e., gives more information) than $q$. We will denote the trivial Boolean algebra by 2 .

For each partial order $\mathbf{P}$, we let $\mathscr{B}(\mathbf{P})$ be the complete Boolean algebra corresponding to $\mathbf{P}$. If $e: \mathbf{P} \hookrightarrow \mathbf{Q}$, then there is a complete monomorphism $i: \mathscr{B}(\mathbf{P}) \rightarrow \mathscr{B}(\mathbf{Q})$ extending $e$. If we define $\pi: \Re(\mathbf{Q}) \rightarrow \Re(\mathbf{P})$ by $\pi(q)=\inf \{p: i(p) \geqslant q\}$, then $\pi$ splits $i$ (and $e$ ) in the sense that $\pi \circ i=$ identity on $\mathscr{B}(\mathbf{P})$. (We will use "id" throughout to 
denote the appropriate identity function whenever necessary.) In this paper we shall use both partial order and Boolean algebra terminology interchangeably. If $\mathscr{B}$ and $\mathcal{C}$

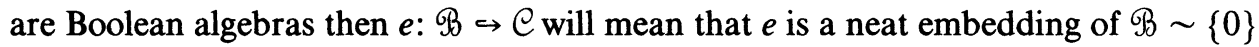
into $C \sim\{0\}$. (Equivalently, $e$ is a complete homomorphism of $\mathscr{B}$ into $\mathcal{C}$.)

The following lemma is standard:

Lemma 2.2. Let $\mathbf{P}$ and $\mathbf{Q}$ be partial orderings. Let $e: \mathbf{P} \rightarrow \mathbf{Q}$ and $\pi: \mathbf{Q} \rightarrow \mathbf{P}$ be order preserving. Suppose

(1) $e$ is one to one,

(2) for all $x \in \mathbf{P}, \pi(e(x))=x$,

(3) for all $y \in \mathbf{Q}, e(\pi(y)) \geqslant y$,

(4) let $y \in \mathbf{Q}, x \in \mathbf{P}$ and $x \leqslant \pi(y)$.

Then there is $a z \leqslant y$ with $\pi(z) \leqslant x$.

Then $e: \mathbf{P} \hookrightarrow \mathbf{Q}$ is a neat embedding and $\pi: \mathbf{Q} \rightarrow \mathbf{P}$ is the restriction of the map $\pi:$ $\Re(\mathbf{Q}) \rightarrow \Re(\mathbf{P})$ splitting $e$.

Suppose $e: \mathbf{P} \hookrightarrow \mathbf{Q}$. Let $S$ be a separative partial ordering in $V^{\mathbf{P}}$. We will define the partial ordering $\mathbf{Q} *_{e} S$. We begin by letting $X$ be the collection of all pairs $\langle q, \tau\rangle$ with

(1) $q \in \mathbf{Q}$ and $\tau \in V^{\mathbf{P}}$,

(2) $\|\tau \in S\|_{\mathbf{P}}=1$ and $\operatorname{rank}(\tau)=\min \left\{\operatorname{rank}\left(\tau^{\prime}\right):\left\|\tau^{\prime}=\tau\right\|_{\mathbf{P}}=1\right\}$. (Rank here is set-theoretical rank.)

Let $\pi: \Re(\mathbf{Q}) \rightarrow \Re(\mathbf{P})$ be the splitting map for $e$. We define an equivalence relation, $\sim$, on $X$ by $(q, \tau) \sim\left(q, \tau^{\prime}\right)$ iff $\pi(q) \mathbb{H}_{\mathscr{B}(\mathbf{P})} \tau=\tau^{\prime}$. The domain of $\mathbf{Q} *_{e} S$ is the set of equivalence classes of $X$ modulo this relation. The ordering on $\mathbf{Q} *_{e} S$ is $\left[\left(q_{1}, \tau_{1}\right)\right]_{\sim} \leqslant$ $\left[\left(q_{2}, \tau_{2}\right)\right]_{\sim}$ iff

(1) $q_{1} \leqslant q_{2}$ in $\mathbf{Q}$

(2) $\pi(q) \mathbb{H}_{\mathscr{B}(\mathbf{P})} \tau_{1} \leqslant \tau_{S} \tau_{2}$.

It is easy for the reader to check that this ordering is well-defined on equivalence classes and gives a separative partial ordering.

In practice we will ignore the fact that we have equivalence classes here. When we mention a condition $\langle q, \tau\rangle$ we will mean its equivalence class and when we discuss an equivalence class we will of ten tacitly be picking a representative of that class. $\mathbf{Q} *_{e} S$ will be called an iteration with amalgamation.

We now state an essential property of iterations with amalgamation:

Lemma 2.3. Let $e: \mathbf{P} \hookrightarrow \mathbf{Q}, f: \mathbf{Q} \hookrightarrow \mathbf{R}$. Let $S \in V^{\mathbf{P}}$ be a partial ordering. Then there is a canonical map $i$ such that the following commutes:

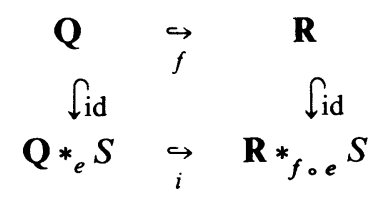

where id is now defined by $\operatorname{id}(q)=(q, 1)$.

Proof. Let $i(q, \tau)=(f(q), \tau)$. It is easy to see that $i$ is one-to-one and order preserving. Let $\pi_{e}, \pi_{f}, \pi_{f \circ e}$ be the maps splitting $e, f$ and $f \circ e$ respectively. 
Let $A \subseteq \mathbf{Q} *{ }_{e} S$ be a maximal antichain; we need to show that $i^{\prime \prime} A$ is a maximal antichain in $\mathbf{R} *_{f_{0} e} S$. Suppose that $(r, \tau) \in \mathbf{R} *_{f_{\circ} e} S$. Let $q \in \mathbf{Q}$ be such that $q \leqslant \pi_{f}(r)$ in $\mathscr{B}(\mathbf{Q})$. Since $A$ is a maximal antichain in $\mathbf{Q}{ }_{e} S$, there is a $\left(q^{\prime}, \tau^{\prime}\right) \in A$ such that $\left(q^{\prime}, \tau^{\prime}\right)$ is compatible with $(q, \tau)$. Pick $\left(q^{\prime \prime}, \tau^{\prime \prime}\right)$ such that $\left(q^{\prime \prime}, \tau^{\prime \prime}\right) \leqslant\left(q^{\prime}, \tau^{\prime}\right)$, $(q, \tau)$. The following claim will suffice:

Claim. (a) $\left(f\left(q^{\prime \prime}\right), \tau^{\prime \prime}\right) \leqslant\left(f\left(q^{\prime}\right), \tau^{\prime}\right)$,

(b) $\left(f\left(q^{\prime \prime}\right), \tau^{\prime \prime}\right)$ is compatible with $(r, \tau)$.

Proof of ClaIM. (a) is true since $i$ is order preserving, (b) is true because $q^{\prime \prime} \leqslant \pi(r)$ so we can pick $r^{\prime} \leqslant f\left(q^{\prime \prime}\right), r$. It is now routine to check that $\left(r^{\prime}, \tau^{\prime \prime}\right) \leqslant(r, \tau)$ using the fact that $\pi_{e} \circ \pi_{f}=\pi_{f \circ e}$.

Assuming the claim we see that $(r, \tau)$ is compatible with $\left(f(q), \tau^{\prime \prime}\right) \in i^{\prime \prime} A$.

We will occasionally use the notation $f *$ id to denote the map $i$ defined above.

3. Some preliminary facts. We start by recalling the Silver modification of the Levy collapse:

Definition. The Silver collapse of $\lambda$ to $\kappa^{+}, S(\kappa, \lambda)$, is the collection of all partial functions $p: \lambda \times \kappa \rightarrow \lambda$ such that

(1) for every $\alpha<\lambda, p \uparrow\{\alpha\} \times \kappa:\{\alpha\} \times \kappa \rightarrow \alpha$,

(2) $|p| \leqslant \kappa$,

(3) There is a $\xi<\kappa$ such that dom $p \subseteq \lambda \times \xi$.

$S(\kappa, \lambda)$ is ordered by reverse inclusion.

By standard arguments one can see that if $\lambda>\kappa$ and $\lambda$ is inaccessible, then $S(\kappa, \lambda)$ is $\lambda$ c.c. and is $<\kappa$-closed. The Silver conditions are not absolute and this causes some important difficulties. To emphasize where we are defining the Silver conditions we will use the notation $S^{\mathbf{Q}}(\kappa, \lambda)$ to denote the Silver conditions defined in $V^{\mathbf{Q}}$. To reduce notational complexity we will write $\mathbf{Q} * \bar{S}(\kappa, \lambda)$ to mean $\mathbf{Q} * S^{\mathbf{Q}}(\kappa, \lambda)$.

We now will define what we will call a master condition. We start with an elementary embedding $j: V \rightarrow M$. (We will not specify how large the embedding is, although in our applications it is at least a huge embedding.) Suppose we have a partial ordering $\mathbf{Q}$ and a neat embedding $i: \mathbf{Q} \hookrightarrow j(\mathbf{Q})$. A master condition for $i$ and $j$ is a condition $p \in j(\mathbf{Q})$ such that for every $q \in \mathbf{Q}$

$$
p \wedge i(q) \leqslant j(q) \text { in } \mathscr{B}(j(\mathbf{Q})) \text {. }
$$

If there is a master condition $p \in j(\mathbf{Q})$ then any generic object $H$ on $j(\mathbf{Q})$ with $p \in H$ has the property that $j^{-1}(H) \subseteq \mathbf{Q}$ is generic. This is because (*) implies that $j^{-1}[H]=i^{-1}[H]$.

THEOREM (KUNEN). Let $j: V \rightarrow M$ be a huge embedding, and $\mathbf{Q}$ be a partial ordering. Suppose there is a map $i$ such that the following diagram commutes:

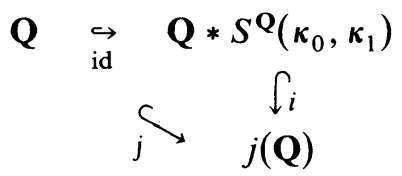

Then there is a master condition for $j$ and the map i. (Viewing $i$ : $\mathbf{Q} * S^{\mathbf{Q}}\left(\kappa_{0}, \kappa_{1}\right) \hookrightarrow$ $j(\mathbf{Q}) * S^{j(\mathbf{Q})}\left(\kappa_{1}, \kappa_{2}\right)$.) 
Proof. We will quickly review the proof of this theorem.

First we remark that $\mathbf{Q}$ is $\kappa_{0}$ c.c., since $j: \mathbf{Q} \hookrightarrow j(\mathbf{Q})$ is a neat embedding. Thus there is no difference between $\mathscr{B}(j(\mathbf{Q}))$ constructed in $M$ and $\mathscr{B}(j(\mathbf{Q}))$ constructed in $V$.

Claim 1. Let $H$ be a canonical term in $V^{j(\mathbf{Q})}$ for a generic object on $j(\mathbf{Q})$. In $V^{j(\mathbf{Q})}$ we consider $S^{j(\mathbf{Q})}\left(\kappa_{1}, \kappa_{2}\right)$. For every $\tau^{\prime} \in S^{j(\mathbf{Q})}\left(\kappa_{1}, \kappa_{2}\right)$ there is a $\tau \in S^{j(\mathbf{Q})}\left(\kappa_{1}, \kappa_{2}\right)$ such that

(1) $\left\|\tau^{\prime}=\tau\right\|_{j(\mathbf{Q})}=1$ computed in $V$,

(2) $\tau \in M^{j(\mathbf{Q})}$,

(3) $\left\|\tau^{V[H]}=\tau^{M[H]}\right\|_{j(\mathbf{Q})}=1$ computed in $V$. (Here, $\tau^{V[H]}$ means the denotation of $\tau$ in $V[H]$ and similarly for $\tau^{M[H]}$.)

By $\kappa_{0}$ c.c. of $\mathbf{Q}$ in $V, j(\mathbf{Q})$ is $\kappa_{1}$ c.c. in $M$ and hence $j(\mathbf{Q})$ is $\kappa_{1}$ c.c. in $V$ since $j$ is a huge embedding. Thus there is a $\xi<\kappa_{1}$ and sets $X, Y \subseteq \kappa_{2}$ with $|X|=\kappa_{1},|Y|=\kappa_{1}$ such that $\left\|\operatorname{dom} \tau^{\prime} \subseteq X \times \xi\right\|_{j(\mathbf{Q})}=1$ and $\|$ range $\tau^{\prime} \subseteq Y \|_{j(\mathbf{Q})}=1$ computed in $V$.

For each triple $\alpha, \beta, \delta$ such that $\alpha \in X, \beta<\xi$ and $\delta \in Y$, let $b_{\alpha, \beta, \delta}=$

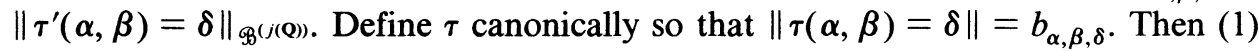
is obvious. (2) is true because $|X \times \xi \times Y|=\kappa_{1}$ and thus $|\tau|=\kappa_{1}$. Since $j$ is huge, $\tau \in M$. (3) is true since $\tau^{\prime}(\alpha, \beta)=\delta$ in $V[G]$ iff $b_{\alpha, \beta, \delta} \in G$, but this is absolute between $V[G]$ and $M[G]$, so $\tau(\alpha, \beta)=\delta$ in $M[G]$.

In $V^{j(\mathbf{Q})}$, we let $G$ be a canonical term for the generic object on $\mathbf{Q} * S^{\mathbf{Q}}\left(\kappa_{0}, \kappa_{1}\right)$ given by $i$. Let $\tau^{\prime}$ be a term in $V^{j(\mathbf{Q})}$ such that $\tau^{\prime}$ denotes

$$
\bigcup_{\substack{\langle q, \sigma\rangle \in G \\ q \in \mathbf{Q} \\ \sigma \in S^{\mathbf{Q}}\left(\kappa_{0}, \kappa_{1}\right)}} j(\sigma)^{M^{j(\mathbf{Q})}},
$$

where $j(\sigma)^{M^{j(\mathbf{Q})}}$ is the denotation of $j(\sigma)$ in $M^{j(\mathbf{Q})}$.

Claim 2. $\tau^{\prime} \in S^{j(\mathbf{Q})}\left(\kappa_{1}, \kappa_{2}\right)$.

Proof. We first check that $\langle q, \sigma\rangle \in G$ and $\left\langle q^{\prime}, \sigma^{\prime}\right\rangle \in G$ implies that $j(\sigma)^{M^{j(\theta)}} \cup$ $j\left(\sigma^{\prime}\right)^{M^{j(\mathbf{Q})}} \in S^{j(\mathbf{Q})}\left(\kappa_{1}, \kappa_{2}\right)$. Let $\langle r, \nu\rangle \leqslant\langle q, \sigma\rangle \wedge\left\langle q^{\prime}, \boldsymbol{\sigma}^{\prime}\right\rangle,\langle r, \nu\rangle \in G$. Then $j(r)$ is in the canonical generic object on $j(\mathbf{Q})$ since $i(\langle r, 1\rangle)=j(r)$ by the diagram. By elementarity of $j, j(r) \mathbb{H}_{j(\mathbf{Q})} j(\sigma) \cup j\left(\sigma^{\prime}\right) \in S\left(\kappa_{1}, \kappa_{2}\right)$.

To see the claim we now need only check the cardinality conditions: In $V^{\mathbf{Q}}$, $\left|S^{\mathbf{Q}}\left(\kappa_{0}, \kappa_{1}\right)\right|=\kappa_{1}$, so in $V^{j(\mathbf{Q})} \mid\left\{(\sigma)^{M^{j(\mathbf{Q})}}\right.$ : there is a $\left.q \in \mathbf{Q},\langle q, \sigma\rangle \in G\right\} \mid=\kappa_{1}$. If $\langle q, \sigma\rangle \in G$, then, in $V^{\mathbf{Q}},|\sigma|=\kappa_{0}$ and thus $|j(\sigma)|=\kappa_{1}$ in $V^{j(\mathbf{Q})}$ by elementarity.

$$
\left|\underset{\substack{\langle q, \sigma\rangle \in G \\ \in \in \mathbf{Q} \\ \sigma(\mathbf{Q})\left(\kappa_{1}, \kappa_{2}\right)}}{\bigcup} j(\sigma)^{M^{j(\mathbf{Q})}}\right|=\kappa_{1} \quad \text { in } V^{j(\mathbf{Q})} .
$$

Given $\langle q, \sigma\rangle \in G$, there is a $q^{\prime} \leqslant q$ with $\left\langle q^{\prime}, \sigma\right\rangle \in G$ such that

$$
q^{\prime} \| \operatorname{dom} \sigma \subseteq \kappa_{1} \times \xi \text { for some } \xi<\kappa_{0} .
$$

So $j\left(q^{\prime}\right) H \operatorname{dom}(j(\sigma)) \subseteq \kappa_{2} \times j(\xi)$. But $j(\xi)=\xi<\kappa_{0}$. Thus,

$$
\operatorname{dom} \bigcup_{\langle q, \sigma\rangle \in G} j(\sigma)^{M^{j(Q)}} \subseteq \kappa_{2} \times \kappa_{0}
$$


and has cardinality $\kappa_{1}$. Hence $\tau^{\prime} \in S^{j(\mathbf{Q})}\left(\kappa_{1}, \kappa_{2}\right)$. Let $\tau \in M^{j(\mathbf{Q})}$ be as in Claim 1 with respect to $\tau^{\prime}$.

Claim 3. $(1, \tau)$ is a master condition for $j$ and $i$.

Proof. Let $\langle q, \sigma\rangle \in \mathbf{Q} * S^{\mathbf{Q}}\left(\kappa_{1}, \kappa_{2}\right)$. We need that $i(q, \sigma) \wedge\langle 1, \tau\rangle \leqslant\langle j(q), j(\sigma)\rangle$. By assumption $i$ : $\mathbf{Q} * S^{\mathbf{Q}}\left(\kappa_{0}, \kappa_{1}\right) \hookrightarrow j(\mathbf{Q})$, so $i(q, \sigma) \wedge\langle 1, \tau\rangle=\langle i(q, \sigma), \tau\rangle$. Now $i(q, \sigma) \leqslant_{j(Q)} i(q, 1)$, but $i(q, 1)=j(q)$ by the diagram. All we have left to check is that $i(q, \sigma) \mathbb{t} \leqslant j(\sigma)$. This is easy because $i(q, \sigma) \mathbb{H}(q, \sigma) \in G$ and thus $i(q, \sigma) \mathbb{H} \tau$ $\leqslant j(\sigma)$, since

$$
\tau=\bigcup_{\left\langle q^{\prime}, \sigma^{\prime}\right\rangle \in G} j\left(\sigma^{\prime}\right) \quad \text { in } V^{j(\mathbf{Q})} .
$$

We will use a variant of this theorem to build master conditions. For reasons to be discussed in the next section, we will have to build master conditions in situations where we do not have closure. The following is a sufficient condition for the existence of a master condition.

Lemma 3.1. Let $\mathbf{P}$ and $\mathbf{Q}$ be partial orderings and $e: \mathbf{P} \hookrightarrow \mathbf{Q}$ be a neat embedding. Let $j: V \rightarrow M$ be a huge embedding. Suppose there are maps $i_{1}$ and $i_{2}$ such that the following diagram commutes:

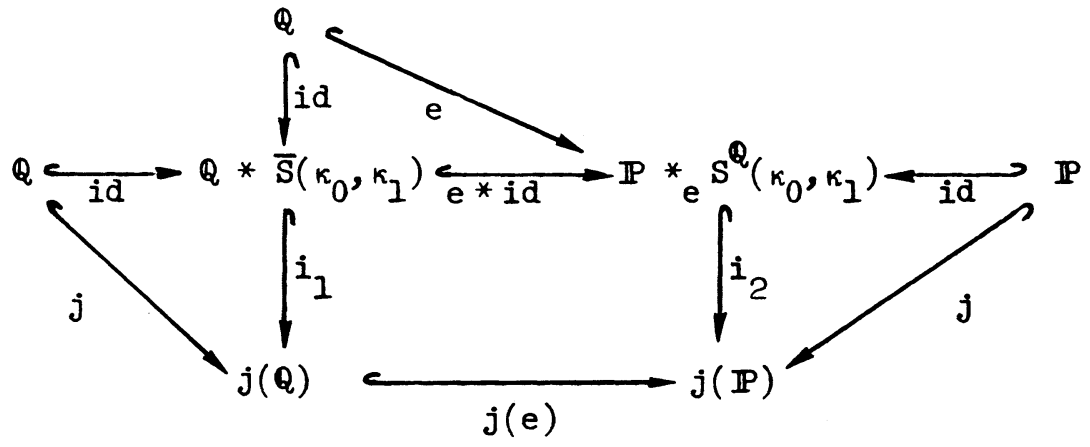

Then there is a master condition for $j$ and

$$
i_{2}: \mathbf{P} *_{e} S^{\mathbf{Q}}\left(\kappa_{0}, \kappa_{1}\right) \hookrightarrow j(\mathbf{P}) *_{j(e)} S^{j(\mathbf{Q})}\left(\kappa_{1}, \kappa_{2}\right) .
$$

Proof. Since the left triangle commutes, the hypotheses of Kunen's theorem are satisfied for $\mathbf{Q}$ and $j(\mathbf{Q})$. Let $\langle 1, \tau\rangle$ be the master condition given by the theorem, with $\tau \in S^{j(\mathbf{Q})}\left(\kappa_{1}, \kappa_{2}\right)$. We claim that $\langle 1, \tau\rangle \in j(\mathbf{P}) * S^{j(\mathbf{Q})}\left(\kappa_{1}, \kappa_{2}\right)$ is a master condition for $j$ and $i_{2}$. We have to check that for any $\langle p, \sigma\rangle \in \mathbf{P} *_{e} S^{\mathbf{Q}}\left(\kappa_{0}, \kappa_{1}\right)$,

$$
i_{2}(p, \sigma) \wedge\langle 1, \tau\rangle \leqslant\langle j(p), j(\sigma)\rangle
$$

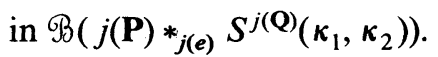

We know that $i_{2}(p, \sigma) \in j(\mathbf{P})$ so $i_{2}(p, \sigma) \wedge\langle 1, \tau\rangle=\left\langle i_{2}(p, \sigma), \tau\right\rangle$. We have to see that $\left\langle i_{2}(p, \sigma), \tau\right\rangle \leqslant(j(p), j(\sigma))$. Now $i_{2}(p, \sigma) \leqslant j(p)$ in $j(\mathbf{P})$, because $i_{2}(p, 1)=$ $j(p)$ and the right-hand triangle commutes. Let $\pi: \mathscr{B}(\mathbf{P}) \rightarrow \mathscr{B}(\mathbf{Q})$ be the map that splits $e$. All we have left to show is that $j(\pi)\left(i_{2}(p, \sigma)\right) H \tau \leqslant j(\sigma)$. We see that 
$(e * \operatorname{id})(\pi(p), \sigma) \leqslant(p, \sigma)$ and thus, since the central square commutes, $i_{1}(\pi(p), \sigma)$ $\geqslant j(\pi)\left(i_{2}(p, \sigma)\right)$. Since $\langle 1, \tau\rangle$ was a master condition for $j$ and $i_{1}$ we get that

$$
\left\langle i_{1}(\pi(p), \sigma), \tau\right\rangle \leqslant\langle j(\pi(p)), j(\sigma)\rangle .
$$

Hence, $i_{1}(\pi(p), \sigma) \mathbb{\tau} \leqslant j(\sigma)$ and thus, $j(\pi)\left(i_{2}(p, \sigma)\right) \Vdash \tau \leqslant j(\sigma)$.

In fact we can prove more: If we let $I_{\langle 1, \tau\rangle}$ be the ideal generated by $\{b$ : $b \wedge\langle 1, \tau\rangle=0\}$ and

$$
\rho: \mathscr{B}\left(j(\mathbf{P}) * S^{j(\mathbf{Q})}\left(\kappa_{1}, \kappa_{2}\right)\right) \rightarrow \mathscr{B}\left(j(\mathbf{P}) * S^{j(\mathbf{Q})}\left(\kappa_{1}, \kappa_{2}\right)\right) / I_{\langle 1, \tau\rangle}
$$

be the projection map, then

$$
\rho \circ j: \mathscr{B}\left(\mathbf{P} * S^{\mathbf{Q}}\left(\kappa_{0}, \kappa_{1}\right)\right) \rightarrow \mathscr{B}\left(j(\mathbf{P}) * S^{j(\mathbf{Q})}\left(\kappa_{1}, \kappa_{2}\right)\right) / I_{\langle 1, \tau\rangle}
$$

is a neat embedding. We leave this to the interested reader.

4. In this section we shall give an overview of the rest of the proof. Our forcing conditions will be of the form $\mathbf{P}\left(\kappa_{0}\right) * \mathbf{R}\left(\kappa_{0}, \kappa_{1}\right) * \bar{S}\left(\kappa_{1}, \kappa_{2}\right)$. Here, $\mathbf{P}\left(\kappa_{0}\right)$ will be a $\kappa_{0}$ c.c. iteration of length $\kappa_{0}$ that collapses $\kappa_{0}$ to $\aleph_{1} . \mathbf{R}\left(\kappa_{0}, \kappa_{1}\right)$ will be a further iteration that collapses $\kappa_{1}$ to $\boldsymbol{\kappa}_{2}$ while adding no new bounded subsets of $\kappa_{0}$ to $V^{\mathbf{P}\left(\kappa_{0}\right)}$. (We will provide a uniform first order definition for partial orders $\mathbf{P}(\alpha) * \mathbf{R}(\alpha, \beta)$ for all Mahlo $\alpha$ and $\beta$.) Finally $\bar{S}\left(\kappa_{1}, \kappa_{2}\right)$ will be the Silver conditions for collapsing $\kappa_{2}$ to $\kappa_{1}^{+}$defined in $V^{\mathbf{P}\left(\kappa_{0}\right)} * \mathbf{R}\left(\kappa_{0}, \kappa_{1}\right)$ and so forcing with $\mathbf{P}\left(\kappa_{0}\right) * \mathbf{R}\left(\kappa_{0}, \kappa_{1}\right) * \bar{S}\left(\kappa_{1}, \kappa_{2}\right)$ makes $\kappa_{2}$ into $\kappa_{3}$.

Unfortunately in order to get a master condition for

$$
j: \mathbf{P}\left(\kappa_{0}\right) * \mathbf{R}\left(\kappa_{0}, \kappa_{1}\right) * \bar{S}\left(\kappa_{1}, \kappa_{2}\right) \rightarrow \mathbf{P}\left(\kappa_{1}\right) * \mathbf{R}\left(\kappa_{1}, \kappa_{2}\right) * \bar{S}\left(\kappa_{2}, \kappa_{3}\right),
$$

the construction of $\mathbf{P}$ and $\mathbf{R}$ has to be rather involved. In order to explicate our construction we list various heuristic requirements that we want $\mathbf{P}$ and $\mathbf{R}$ to satisfy and indicate how we go about satisfying them. In an attempt at clarity and sometimes at the expense of total accuracy we will simplify somewhat what the difficulties and solutions are.

(1) We can view our master condition $p$ as being $\left(p_{1}, \sigma\right)$ where $p_{1} \in$ $\mathbf{P}\left(\kappa_{1}\right) * \mathbf{R}\left(\kappa_{1}, \kappa_{2}\right)$ and $\sigma$ is a term in $V^{\mathbf{P}\left(\kappa_{1}\right)} * \mathbf{R}\left(\kappa_{1}, \kappa_{2}\right)$ for an element of

$$
S^{\mathbf{P}\left(\kappa_{1}\right) * \mathbf{R}\left(\kappa_{1}, \kappa_{2}\right)}\left(\kappa_{2}, \kappa_{3}\right) .
$$

The condition $p_{1} \in \mathbf{P}\left(\kappa_{1}\right) * \mathbf{R}\left(\kappa_{1}, \kappa_{2}\right)$ will be a master condition for

$$
j: \mathbf{P}\left(\kappa_{0}\right) * \mathbf{R}\left(\kappa_{0}, \kappa_{1}\right) \rightarrow \mathbf{P}\left(\kappa_{1}\right) * \mathbf{R}\left(\kappa_{1}, \kappa_{2}\right) .
$$

In order to use the Kunen approach to build the second component, $\sigma$, of our master condition, we need that

$$
\mathbf{P}\left(\kappa_{0}\right) * \mathbf{R}\left(\kappa_{0}, \kappa_{1}\right) * \bar{S}\left(\kappa_{1}, \kappa_{2}\right) \hookrightarrow \mathbf{P}\left(\kappa_{1}\right) * \mathbf{R}\left(\kappa_{1}, \kappa_{2}\right) .
$$

In our construction we build a canonical map icon ${ }^{\left(\kappa_{0}, \kappa_{1}\right)}: \mathbf{P}\left(\kappa_{0}\right) * \mathbf{R}\left(\kappa_{0}, \kappa_{1}\right) \Leftrightarrow \mathbf{P}\left(\kappa_{1}\right)$ and extend icon $^{\left(\kappa_{0}, \kappa_{1}\right)}$ to a map

$$
\mathbf{P}\left(\kappa_{0}\right) * \mathbf{R}\left(\kappa_{0}, \kappa_{1}\right) * \bar{S}\left(\kappa_{1}, \kappa_{2}\right) \hookrightarrow \mathbf{P}\left(\kappa_{1}\right) * \mathbf{R}\left(\kappa_{1}, \kappa_{2}\right),
$$

by making

$$
S^{\mathbf{P}\left(\kappa_{0}\right) * \mathbf{R}\left(\kappa_{0}, \kappa_{1}\right)}\left(\kappa_{1}, \kappa_{2}\right)
$$


a stage in the iteration $\mathbf{R}\left(\kappa_{1}, \kappa_{2}\right)$. (Again $\operatorname{icon}^{(\alpha, \beta)}$ will be uniformly defined with parameters $\alpha$ and $\beta$.)

(2) By the elementarity of $j$ and requirement (1) we find that for almost all $\alpha<\kappa_{0}$ we must have a map $\varphi_{\alpha}$

$$
\varphi_{\alpha}: \mathbf{P}(\alpha) * \mathbf{R}\left(\alpha, \kappa_{0}\right) * \bar{S}\left(\kappa_{0}, \kappa_{1}\right) \rightarrow \mathbf{P}\left(\kappa_{0}\right) * \mathbf{R}\left(\kappa_{0}, \kappa_{1}\right)
$$

and

$$
S^{\mathbf{P}(\alpha) * \mathbf{R}\left(\alpha, \kappa_{0}\right)}\left(\kappa_{0}, \kappa_{1}\right)
$$

is a stage in the iteration $\mathbf{R}\left(\kappa_{0}, \kappa_{1}\right)$.

We shall now attempt to describe our strategy for building the master condition $p_{1}$ for $j: \mathbf{P}\left(\kappa_{0}\right) * \mathbf{R}\left(\kappa_{0}, \kappa_{1}\right) \rightarrow \mathbf{P}\left(\kappa_{1}\right) * \mathbf{R}\left(\kappa_{1}, \kappa_{2}\right)$. If $\mathbf{Q} \hookrightarrow_{e} \mathbf{P}$, Lemma 3.1 gives a way to build a master condition for

$$
j: \mathbf{P} *_{e} S^{\mathbf{Q}}\left(\kappa_{0}, \kappa_{1}\right) \rightarrow j(\mathbf{P}) *_{j(e)} S^{j(\mathbf{Q})}\left(\kappa_{1}, \kappa_{2}\right) .
$$

Suppose we have $\mathbf{Q}_{1} \hookrightarrow_{e_{1}} \mathbf{P}$ and $\mathbf{Q}_{2} \hookrightarrow_{e_{2}} \mathbf{P}$ satisfying Lemma 3.1 and master conditions

$$
\left(1, q_{1}\right) \in j(\mathbf{P}) *_{j\left(e_{2}\right)} S^{j\left(\mathbf{Q}_{1}\right)}\left(\kappa_{1}, \kappa_{2}\right)
$$

and

$$
\left(1, q_{2}\right) \in j(\mathbf{P}) *_{j\left(e_{1}\right)} S^{j\left(\mathbf{Q}_{2}\right)}\left(\kappa_{1}, \kappa_{2}\right)
$$

for

$$
j: \mathbf{P} *_{e_{1}} S^{j\left(\mathbf{Q}_{1}\right)}\left(\kappa_{0}, \kappa_{1}\right) \rightarrow j(\mathbf{P}) *_{j\left(e_{1}\right)} S^{j\left(\mathbf{Q}_{1}\right)}\left(\kappa_{1}, \kappa_{2}\right)
$$

and

$$
j: \mathbf{P} *_{e_{2}} S^{j\left(\mathbf{Q}_{2}\right)}\left(\kappa_{0}, \kappa_{1}\right) \rightarrow j(\mathbf{P}) *_{j\left(e_{2}\right)} S^{j\left(\mathbf{Q}_{2}\right)}\left(\kappa_{1}, \kappa_{2}\right)
$$

respectively. We find that we can put $q_{1}$ and $q_{2}$ together to form a condition

$$
\left(1, q_{1}, q_{2}\right) \in j(\mathbf{P}) *_{j\left(e_{1}\right)} S^{j\left(\mathbf{Q}_{1}\right)}\left(\kappa_{1}, \kappa_{2}\right) *_{j\left(\mathrm{id} \circ e_{2}\right)} S^{j\left(\mathbf{Q}_{2}\right)}\left(\kappa_{1}, \kappa_{2}\right)
$$

and this works as a master condition for

$$
\begin{aligned}
j: \mathbf{P} *_{e_{1}} S^{\mathbf{Q}_{1}}\left(\kappa_{0}, \kappa_{1}\right) *_{\text {id } \circ e_{2}} & S^{\mathbf{Q}_{2}}\left(\kappa_{0}, \kappa_{1}\right) \\
& \rightarrow j(\mathbf{P}) *_{j\left(e_{1}\right)} S^{j\left(\mathbf{Q}_{1}\right)}\left(\kappa_{1}, \kappa_{2}\right) *_{j\left(\text { id } \circ e_{2}\right)} S^{j\left(\mathbf{Q}_{2}\right)}\left(\kappa_{1}, \kappa_{2}\right) .
\end{aligned}
$$

What we have done is observe that if we have a master condition for each stage of an iteration we can put them together to get a master condition for the iteration. This works for iterations of infinite length as well (providing we have hospitable supports) and is the key to our master condition construction.

In every coordinate corresponding to an $\alpha$ where

$$
\varphi_{\alpha}: \mathbf{P}(\alpha) * \mathbf{R}\left(\alpha, \kappa_{0}\right) * \bar{S}\left(\kappa_{0}, \kappa_{1}\right) \rightarrow \mathbf{P}\left(\kappa_{0}\right) * \mathbf{R}\left(\kappa_{0}, \kappa_{1}\right),
$$

we must be able to build a master condition. We do this by satisfying the following requirement, which is a variation of the diagram in Lemma 3.1. 
(3) For almost all $\alpha$ there is a map $k_{1}$, making the following diagram commute:

$$
\begin{array}{ccc}
\mathbf{P}(\alpha) * \mathbf{R}\left(\alpha, \kappa_{0}\right) * \bar{S}\left(\kappa_{0}, \kappa_{1}\right) & \underset{\varphi_{\alpha}}{\hookrightarrow} & \mathbf{P}\left(\kappa_{0}\right) * \mathbf{R}\left(\kappa_{0}, \kappa_{1}\right) \\
\int_{k_{1}} & & \int_{\text {icon }}\left(\kappa_{0}, \kappa_{1}\right) \\
\mathbf{P}(\alpha) * \mathbf{R}\left(\alpha, \kappa_{1}\right) & \underset{\operatorname{icon}^{\left(\alpha, \kappa_{1}\right)}}{\hookrightarrow} & \mathbf{P}\left(\kappa_{1}\right)
\end{array}
$$

From (3) we can again infer some elements of our construction. First of all from the existence of $k_{1}$ we see that:

(4) For all relevant $\alpha$, for almost all $\beta$ :

$$
\mathbf{P}(\alpha) * \mathbf{R}(\alpha, \beta) * \bar{S}\left(\beta, \kappa_{0}\right) \hookrightarrow \mathbf{P}(\alpha) * \mathbf{R}\left(\alpha, \kappa_{0}\right) .
$$

We make this happen in a straightforward manner similar to Kunen's construction, namely, we build $\mathbf{P}(\alpha) * \mathbf{R}\left(\alpha, \kappa_{0}\right)$ as an iteration of length $\kappa_{0}$ taking steps to make sure that whenever

$$
\mathbf{P}(\alpha) * \mathbf{R}(\alpha, \beta) \underset{e}{\rightarrow} \mathbf{P}(\alpha) * \mathbf{R}_{\gamma}\left(\alpha, \kappa_{0}\right)
$$

for some $\gamma$, there is a stage $\xi<\kappa_{0}$ such that

$$
\mathbf{P}(\alpha) * \mathbf{R}_{\xi+1}\left(\alpha, \kappa_{0}\right)=\mathbf{P}(\alpha) * \mathbf{R}_{\xi}\left(\alpha, \kappa_{0}\right) *_{e} S^{\mathbf{P}(\alpha) * \mathbf{R}(\alpha, \beta)}\left(\beta, \kappa_{0}\right) .
$$

(Here $\mathbf{P}(\alpha) * \mathbf{R}_{\gamma}\left(\alpha, \kappa_{0}\right)$ is the $\gamma$ th stage in the iteration creating $\mathbf{P}(\alpha) * \mathbf{R}\left(\alpha, \kappa_{0}\right)$.)

(5) $\mathbf{P}(\alpha)$ will be an $\alpha$ stage iteration designed to take into account these requirements. As we build $\mathbf{P}(\alpha)$ we will also be building the maps icon ${ }^{(\beta, \alpha)}: \mathbf{P}(\beta) * \mathbf{R}(\beta, \alpha)$ $\Leftrightarrow \mathbf{P}(\alpha)$. Roughly for one iteration to be embedded in another it is enough that there be coordinate by coordinate embeddings and that the supports of the two iterations be compatible. One informal way of viewing the map icon ${ }^{(\beta, \alpha)}$ is to think of building $\mathbf{P}(\beta) * \mathbf{R}(\beta, \alpha)$ and $\mathbf{P}(\alpha)$ through time.

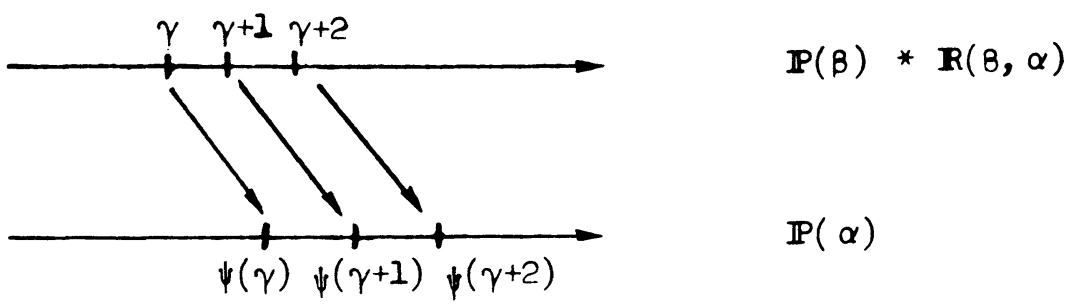

For each time $\gamma$ (a stage of the iteration) in $\mathbf{P}(\beta) * \mathbf{R}(\beta, \alpha)$ we associate a time (or stage) $\psi(\gamma)$ in $\mathbf{P}(\alpha)$. At time $\psi(\gamma)$ we will inductively have built a map

$$
\operatorname{icon}_{\gamma}^{(\beta, \alpha)}: \mathbf{P}(\beta) * \mathbf{R}_{\gamma}(\beta, \alpha) \hookrightarrow \mathbf{P}_{\psi(\gamma)}(\alpha) .
$$

Suppose that $\mathbf{P}(\beta) * \mathbf{R}_{\gamma+1}(\beta, \alpha)=\mathbf{P}(\beta) * \mathbf{R}_{\gamma}(\beta, \alpha) *_{e} S_{\gamma}^{\mathbf{Q}_{\gamma}}$ for some $e_{\gamma}: \mathbf{Q}_{\gamma} a$ $\mathbf{P}(\beta) * \mathbf{R}_{\gamma}(\beta, \alpha)$ and $S_{\gamma}$, then to continue the embedding one more stage we make

$$
\mathbf{P}_{\psi(\gamma)+1}(\boldsymbol{\alpha})=\mathbf{P}_{\psi(\gamma)}{ }^{*}{ }_{\text {icon }}^{(\beta, \alpha) \circ e_{\gamma}} S_{\gamma}^{\mathbf{Q}_{\gamma}} .
$$


Using Lemma 2.3 we can prolong to icon ${ }_{\gamma+1}^{(\beta, \alpha)}: \mathbf{P}(\beta) * \mathbf{R}_{\gamma+1}(\beta, \alpha) \rightarrow \mathbf{P}_{\psi(\gamma)+1}(\alpha)$. In order to carry this process through limit stages we need some support criterion. This is developed in $\S 5$.

One way of thinking about this is that what happens at stage $\gamma$ in building $\mathbf{P}(\beta) * \mathbf{R}(\beta, \alpha)$ induces a requirement on $\mathbf{P}(\alpha)$ which we handle at time $\psi(\gamma)$. This is a useful heuristic when trying to make diagrams commute. In a typical situation we are concerned about, we will be given a commutative diagram:

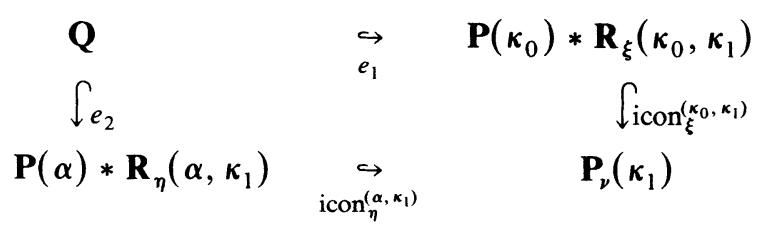

for some $\mathbf{Q}, e_{1}$ and $e_{2}$. We will want to make the following diagram commute for some $i_{1}$ and $i_{2}$ :

$$
\begin{array}{rcc}
\mathbf{Q} * \bar{S}\left(\kappa_{0}, \kappa_{1}\right) & \underset{e_{1} * i_{1}}{\hookrightarrow} & \mathbf{P}\left(\kappa_{0}\right) * \mathbf{R}\left(\kappa_{0}, \kappa_{1}\right) \\
\int_{e_{2} * i_{2}} & & \int_{\mathrm{icon}}^{\left(\kappa_{0}, \kappa_{1}\right)} \\
\mathbf{P}(\alpha) * \mathbf{R}\left(\alpha, \kappa_{1}\right) & \underset{\operatorname{icon}^{\left(\alpha, \kappa_{1}\right)}}{\hookrightarrow} & \mathbf{P}\left(\kappa_{1}\right) .
\end{array}
$$

The way we build $e_{1} * i_{1}$ and $e_{2} * i_{2}$ is to create stages $\xi_{1}>\xi$ and $\eta_{1}>\eta$ where

$$
\mathbf{P}\left(\kappa_{0}\right) * \mathbf{R}_{\xi_{1}+1}\left(\kappa_{0}, \kappa_{1}\right)=\mathbf{P}\left(\kappa_{0}\right) * \mathbf{R}_{\xi_{1}}\left(\kappa_{0}, \kappa_{1}\right) *_{e_{1}} S^{\mathbf{Q}}\left(\kappa_{0}, \kappa_{1}\right)
$$

and

$$
\mathbf{P}(\alpha) * \mathbf{R}_{\eta_{1}+1}\left(\alpha, \kappa_{1}\right)=\mathbf{P}(\alpha) * \mathbf{R}_{\eta_{1}}\left(\alpha, \kappa_{1}\right) *_{e_{2}} S^{\mathbf{Q}}\left(\kappa_{0}, \kappa_{1}\right) .
$$

By Lemma 2.3 we get natural embeddings $e_{1} * i_{1}$ and $e_{2} * i_{2}$ that extend $e_{1}$ and $e_{2}$.

This induces two requirements on the construction of $\mathbf{P}\left(\kappa_{1}\right)$,, namely that we reserve some time, which we will call $\psi_{1}\left(\xi_{1}\right)$ where

$$
\mathbf{P}_{\psi_{1}\left(\xi_{1}\right)+1}\left(\kappa_{1}\right)=\mathbf{P}_{\psi_{1}\left(\xi_{1}\right)}\left(\kappa_{1}\right) *_{\text {icon } \xi_{1}\left(\kappa_{1}, \kappa_{1}\right) \circ e_{1}} S^{\mathbf{Q}}\left(\kappa_{0}, \kappa_{1}\right)
$$

(this corresponds to the requirement induced by

$$
\left.\mathbf{P}\left(\kappa_{0}\right) * \mathbf{R}_{\xi_{1}+1}\left(\kappa_{0}, \kappa_{1}\right)=\mathbf{P}\left(\kappa_{0}\right) * \mathbf{R}_{\xi_{1}}\left(\kappa_{0}, \kappa_{1}\right) *_{e_{1}} S^{\mathbf{Q}}\left(\kappa_{0}, \kappa_{1}\right)\right)
$$

and some time $\psi_{2}\left(\eta_{1}\right)$ where

$$
\mathbf{P}_{\psi_{2}\left(\eta_{1}\right)+1}\left(\kappa_{1}\right)=\mathbf{P}_{\psi_{2}\left(\eta_{1}\right)}\left(\kappa_{1}\right) *_{\text {icon }_{\eta_{1}}^{\left(\alpha, \alpha_{1}\right)} \circ e_{2}} S^{\mathbf{Q}}\left(\kappa_{0}, \kappa_{1}\right) .
$$

We note that if $\gamma>\max \left(\psi_{1}(\xi), \psi_{2}(\eta)\right)$ then

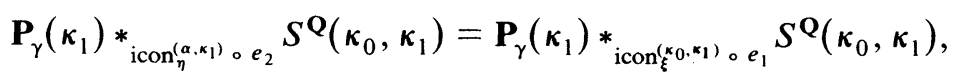

since the given diagram commutes. Thus we can satisfy both of these requirements at the same time by letting $\psi_{1}\left(\xi_{1}\right)=\psi_{2}\left(\xi_{2}\right)=\gamma$ for some $\gamma>\max \left(\psi_{1}(\xi), \psi_{2}(\eta), \xi_{1}, \eta_{1}\right)$. If we make this happen while we are building the maps $\operatorname{icon}^{\left(\alpha, \kappa_{0}\right)}$ and $\operatorname{icon}^{\left(\kappa_{0}, \kappa_{1}\right)}$ then both maps send the coordinate corresponding to $S^{\mathbf{Q}}\left(\kappa_{0}, \kappa_{1}\right)$ in $\mathbf{P}(\alpha) * \mathbf{R}\left(\alpha, \kappa_{1}\right)$ and 
$\mathbf{P}\left(\kappa_{0}\right) * \mathbf{R}\left(\kappa_{0}, \kappa_{1}\right)$ to the same coordinate in $\mathbf{P}\left(\kappa_{1}\right)$. When this is untangled we find that we have made the desired diagram commute.

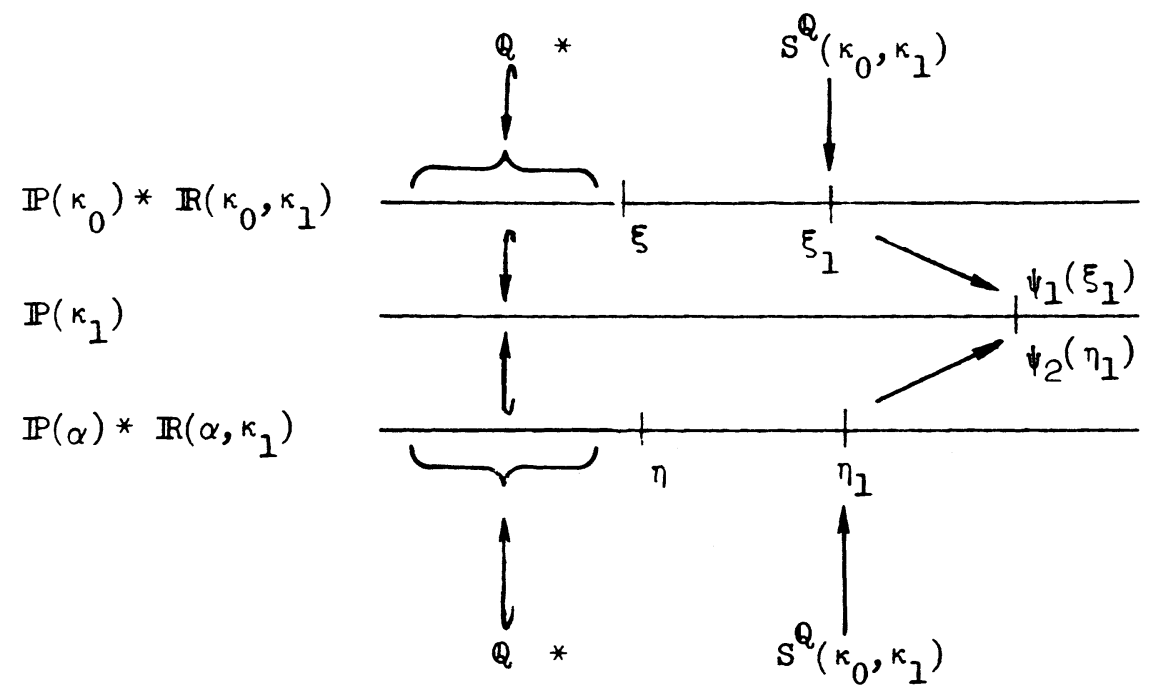

Timing is of the essence in doing this. The construction requires a lot of waiting before we can pick $\gamma$. Typically we shall consider

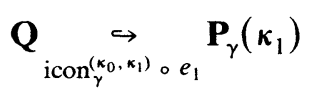

for many $\gamma$, waiting until several conditions are satisfied before setting $\psi_{1}\left(\xi_{1}\right)=\gamma=$ $\psi_{2}\left(\eta_{1}\right)$.

(6) A word is in order as to the logical sequence in building the P's and R's. The requirements above are somewhat circular in the sense that we want to build the R's to accommodate the P's and the P's to accommodate the R's. The way we get around this is to build for each $\mathbf{Q} \subseteq V_{\lambda}, \mathbf{Q} \lambda$ c.c. an object we call $\mathbf{Q} * \mathbf{R}(\lambda, \kappa)$. (There are other parameters in the construction but we omit them here for simplicity.) We can view this as a process for going from a partial ordering $\mathbf{Q}$ to $\mathbf{Q} * \mathbf{R}(\lambda, \kappa)$.

$\mathbf{P}(\alpha)$ is then defined in terms of this "process" $\mathbf{R}$. We can do this one way by making $\mathbf{P}(\alpha)=S(\omega, \alpha)$ if $\alpha$ is the first Mahlo cardinal. Then to define $\mathbf{P}(\alpha)$ for larger $\alpha$, we assume by induction that for all $\beta<\alpha, \mathbf{P}(\beta)$ is defined. Using our process $\mathbf{R}$ we can define $\mathbf{P}(\beta) * \mathbf{R}(\beta, \alpha)$ and in turn use these partial orderings to build $\mathbf{P}(\alpha)$. (Another way is to use a fixed point construction reminiscent of the recursion theorem.) In both iterations $\mathbf{P}(\alpha)$ and $\mathbf{P}(\beta) * \mathbf{R}(\beta, \alpha)$, every stage is the Silver collapse defined in $V^{\mathbf{Q}}$ for some $\mathbf{Q}$.

For each $\mathbf{Q} \subseteq V_{\lambda}, \mathbf{Q} \lambda$ c.c., $\mathbf{Q} * \mathbf{R}(\lambda, \kappa)$ is built in two phases. The first phase roughly corresponds to requirement (2). This phase will be an iteration with support of cardinality $<\lambda$. The second phase will correspond to requirement (4). We will use supports of cardinality $\lambda$ for this phase. The difference in supports is for technical reasons in building the master condition. 
5. We will build our partial orderings $\mathbf{P}(\alpha)$ and $\mathbf{R}(\alpha, \beta)$ as special kinds of iterations. While doing so we will also be building maps to make various diagrams commute.

Consider a partial ordering $\mathbf{Q}$ in the ground model. We may have two iterations $\mathbf{P}$ and $\mathbf{R}$ with $\mathbf{Q}$ occurring as a factor in both iterations. Suppose $\alpha$ and $\beta$ are such that $\mathbf{R}_{\alpha+1}=\mathbf{R}_{\alpha} * \mathbf{Q}$ and $\mathbf{P}_{\beta+1}=\mathbf{P}_{\beta} * \mathbf{Q}$. If we have a map $\varphi_{\alpha \beta}: \mathbf{R}_{\alpha} \rightarrow \mathbf{P}_{\beta}$ then by Lemma 2.3 there is a map $\varphi^{*}$ extending $\varphi_{\alpha \beta}$ such that

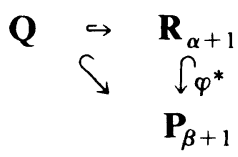

If every factor of $\mathbf{R}$ occurs in this way as a factor of $\mathbf{P}$ and supports are compatible then we can find a map $\varphi: \mathbf{R} \hookrightarrow \mathbf{P}$ extending $\varphi^{*}$. We now develop the technology to do this:

We define by induction on $\lambda$ an ordinal, for every ideal $\mathscr{K}$ on $\lambda$ containing all finite subsets of $\lambda$, what an iteration of length $\lambda$ with support $\mathscr{K}$ is. Suppose we have defined what an iteration of length $\alpha$ with support $\mathscr{K}^{\prime}$ is for all $\alpha<\lambda$ and all $\mathcal{K}^{\prime}$ an ideal on $\alpha$.

Consider the situation where we are given $\left\langle\mathscr{K}_{\alpha}: \alpha<\lambda\right\rangle$ and a $\mathscr{K}$ such that $\mathcal{K}_{\alpha}=\langle X \cap \alpha: X \in \mathscr{K}\rangle$, and we have partial orderings $\left\langle\mathbf{P}_{\alpha}: \alpha<\lambda\right\rangle$ and triples $\left\langle\left(Q_{\alpha}, e_{\alpha}, S_{\alpha}\right): \alpha<\lambda\right\rangle$ such that:

(1) $e_{\alpha}: Q_{\alpha} \Rightarrow \mathbf{P}_{\alpha}$ and $Q_{\alpha}$ H $S_{\alpha}$ is a partial order.

(2) for every $\alpha, \mathbf{P}_{\alpha+1}=\mathbf{P}_{\alpha} *_{e_{\alpha}} S_{\alpha}$.

(3) for every $\alpha<\beta, \mathbf{P}_{\alpha} \rightarrow \mathbf{P}_{\beta}$.

(4) at limit stages $\alpha, \mathbf{P}_{\alpha}$ is an iteration of length $\alpha$ with support $\mathcal{K}_{\alpha}$.

Let $X=\{p: p$ is a function with domain $\lambda$ and for all $\alpha$

(a) $p(\alpha) \in V^{\mathbf{Q}_{\alpha}}$ and $\left\|p(\alpha) \in S_{\alpha}\right\|_{\mathbf{Q}_{\alpha}}=1$,

(b) $\left.\left\{\alpha: p \nmid \alpha H_{\mathrm{P}_{\alpha}} p(\alpha)=1\right\} \in \mathscr{K}\right\}$.

We put an equivalence relation $\sim$ on $X$ by $p_{1} \sim p_{2}$ iff for every $\alpha, p_{1} \mid \alpha H p_{1}(\alpha)=$ $p_{2}(\alpha)$. The iteration of length $\lambda$ with support $\mathscr{K}, \mathbf{P}_{\lambda}$ is the collection of these equivalence classes. The ordering is defined by $\left[p_{1}\right] \leqslant\left[p_{2}\right]$ iff for every $\alpha, p_{1} \mid \alpha H$ $p_{1}(\alpha) \leqslant p_{2}(\alpha)$.

It is left to the reader to see that $\mathbf{P}_{\lambda}$ is a well-defined, separative partial ordering. As before we will often blur the distinction between equivalence classes and their representatives. Given a condition $[p] \in \mathbf{P}_{\alpha}, p$ is a function with domain $\alpha$. We can view it as a function $p^{\prime}$ with domain $\lambda$ by defining it to be $1_{S_{\beta}}$ for all $\alpha \leqslant \beta<\lambda$. The map $p \mapsto p^{\prime}$ is well-defined on equivalence classes and thus induces a map, which we will call id: $\mathbf{P}_{\alpha} \rightarrow \mathbf{P}_{\lambda}$. For a condition $p$, we will call the support of $p$ (notation: supp $p$ ), the collection of all $\beta$ such that $p$ | $\beta H p(\beta)=1$. We will often make no distinction between a condition $p$ in $\mathbf{P}_{\lambda}$ as a function on $\lambda$ and as a function on its support, i.e., given a partial function with domain included in $\lambda$ we can view it as an element of $\mathbf{P}_{\lambda}$ by filling in 1's everywhere it is not defined. In this way we can construe an element of $\mathbf{P}_{\alpha}$ as an element of $\mathbf{P}_{\lambda}$. 
Given $[p] \in \mathbf{P}_{\lambda}$ and $\alpha<\lambda$ we consider $p \nmid \alpha$. The map $\pi_{\alpha}(p)=p \nmid \alpha$ is well defined on equivalence classes in $\mathbf{P}_{\lambda}$. It is now easy to check that id: $\mathbf{P}_{\alpha} \rightarrow \mathbf{P}_{\lambda}$ and $\pi_{\alpha}$ : $\mathbf{P}_{\lambda} \rightarrow \mathbf{P}_{\alpha}$ satisfies the criterion of Lemma 2.2. Thus id: $\mathbf{P}_{\alpha} \hookrightarrow \mathbf{P}_{\lambda}$.

In practice, our iterations will be built inductively. We will have $\left\langle\mathscr{K}_{\alpha}: \alpha<\lambda\right\rangle$ constructed already and choose $\mathscr{K}$ at that point so that $\mathcal{K}_{\alpha}=\{x \cap \alpha: x \in \mathcal{K}\}$. If $\mathscr{K}=\cup_{\alpha<\lambda} \mathcal{K}_{\alpha}$ we call the iteration with support $\mathscr{K}$ of length $\lambda$ the direct limit of $\left\langle\mathbf{P}_{\alpha}: \alpha<\lambda\right\rangle, \lim \mathbf{P}_{\alpha}$. The inverse limit of $\left\langle\mathbf{P}_{\alpha}: \alpha<\lambda\right\rangle, \lim \mathbf{P}_{\alpha}$, is defined by $\mathscr{K}=\{x$ : for all $\left.\alpha<\lambda, \vec{x} \cap \alpha \in \mathscr{K}_{\alpha}\right\}$.

LEMMA 5.1. Let $\mathbf{R}=\mathbf{R}_{\kappa}$ and $\mathbf{P}=\mathbf{P}_{\lambda}$ be iterations of length $\kappa$ and $\lambda$ with supports $\mathcal{K}$ and $\mathscr{K}^{\prime}$ respectively. Let $\psi: \kappa \rightarrow \lambda$ be a monotone function.

Suppose:

(a) for every $\alpha<\kappa$ we have neat embeddings $\varphi_{\alpha}: \mathbf{R}_{\alpha} \hookrightarrow \mathbf{P}_{\psi(\alpha)}$ with

$$
\begin{gathered}
\mathbf{R}_{\alpha} \hookrightarrow \mathbf{R}_{\beta} \\
\varphi_{\alpha} \subseteq \int \varphi_{\beta} \\
\mathbf{P}_{\lambda}
\end{gathered}
$$

(b) for every $\alpha$, if $Q_{\alpha} \underset{e_{\alpha}}{\hookrightarrow} \mathbf{R}_{\alpha}$ and $\mathbf{R}_{\alpha+1}=\mathbf{R}_{\alpha}{ }^{*} e_{\alpha} S_{\alpha}$, then

$$
\mathbf{P}_{\psi(\alpha)+1}=\mathbf{P}_{\psi(\alpha)}{ }^{*}{ }_{\varphi_{\alpha} \circ e_{\alpha}} S_{\alpha}
$$

and $\varphi_{\alpha+1}$ is the canonical map making

$$
\begin{array}{ccc}
\mathbf{R}_{\alpha} & \hookrightarrow & \mathbf{R}_{\alpha+1} \\
\int_{\varphi_{\alpha}} & & \int_{\varphi_{\alpha+1}} \\
\mathbf{P}_{\psi(\alpha)} & \stackrel{\text { id }}{ } & \mathbf{P}_{\psi(\alpha)+1}
\end{array}
$$

commute;

(c) $X \in \mathscr{K}$ iff $\psi^{\prime \prime} X \in \mathscr{K}^{\prime}$;

(d) for all $r \in \mathbf{R}_{\alpha}$, supp $\varphi_{\alpha}(r)=\operatorname{supp} \varphi_{0}(r(0)) \cup \psi^{\prime \prime} \operatorname{supp} r$.

Then there is a canonical neat embedding $\varphi: \mathbf{R}_{\kappa} \Leftrightarrow \mathbf{P}_{\lambda}$ making the following diagram commute

$$
\underset{\varphi_{\alpha} \int_{\mathbf{P}_{\lambda}}^{\stackrel{\mathbf{R}_{\alpha}}{\hookrightarrow} \mathbf{R}_{\kappa}} \Omega_{\varphi}}{ }
$$

Proof. For $r \in \mathbf{R}$, let $X=\operatorname{supp} r$. Then $X \in \mathscr{K}$ and we can consider $r$ as $\langle r(\alpha)$ : $\alpha \in X\rangle$. Let $\varphi(r)=p$ where $p$ is the condition with support $\operatorname{supp} \varphi_{0}(p(0)) \cup \psi^{\prime \prime} X$ defined by

$$
\begin{aligned}
p \uparrow \psi(0) & =\varphi_{0}(r(0)), \\
p(\psi(\alpha)) & =r(\alpha) .
\end{aligned}
$$

$p$ is a condition since supp $\varphi_{0}(r(0)) \cup \psi^{\prime \prime} X \in \mathscr{K}^{\prime}$ by (c).

Note that by clauses (b) and (d), $\varphi(p \nmid \alpha)=\varphi_{\alpha}(p \nmid \alpha)$ for any $\alpha$. This is an easy induction and is left to the reader. 
Claim 1. $\varphi: \mathbf{R}_{\boldsymbol{\kappa}} \rightarrow \mathbf{P}_{\lambda}$ is order-preserving.

Proof. Let $r_{1} \leqslant r_{2}$ and $p_{1}=\varphi\left(r_{1}\right), p_{2}=\varphi\left(r_{2}\right)$. We must show that $p_{1} \leqslant p_{2}$. First, $p_{1} \mid \psi(0) \leqslant p_{2} \uparrow \psi(0)$ since $\varphi_{0}$ is order-preserving. Suppose by induction that $p_{1} \mid \beta \leqslant$ $p_{2} \uparrow \beta$ for all $\beta<\alpha$. If $\alpha$ is a limit then it is trivial that $p_{1}\left|\alpha \leqslant p_{2}\right| \alpha$. If $\alpha=\beta+1$ for some $\beta \in \operatorname{supp} p_{1} \cup \operatorname{supp} p_{2}$ then $\beta=\psi(\gamma)$ for some $\gamma$. Now by clause (b),

$$
\mathbf{P}_{\psi(\gamma)+1}=\mathbf{P}_{\psi(\gamma)} *_{\varphi_{\gamma} \circ e_{\gamma}} S_{\gamma}
$$

and

$$
p_{1} \backslash \alpha=\varphi_{\gamma+1}\left(r_{1} \uparrow \gamma+1\right), \quad p_{2} \uparrow \alpha=\varphi_{\gamma+1}\left(r_{2} \uparrow \gamma+1\right) .
$$

Since $\varphi_{\gamma+1}$ is order preserving and $r_{1}\left|\gamma+1 \leqslant r_{2}\right| \gamma+1, p_{1}\left|\alpha \leqslant p_{2}\right| \alpha$.

ClaIM 2. If $A \subseteq \mathbf{R}_{\kappa}$ is a maximal antichain, then $\varphi^{\prime \prime} A$ is a maximal antichain in $\mathbf{P}_{\lambda}$.

Proof. Let $p \in \mathbf{P}_{\lambda}$ be incompatible with every element of $\varphi^{\prime \prime} A$. Let $\left\langle\pi_{\alpha}: \alpha<\kappa\right\rangle$ be the sequence of splitting maps of $\left\langle\varphi_{\alpha}: \alpha<\kappa\right\rangle$. Pick $q(0) \in \mathbf{R}_{0}, q(0)<$ $\pi_{0}(p \mid \psi(0))$. Let $q=(0, q(0)) \cup\langle(\alpha, p(\psi(\alpha))): \alpha<\kappa\rangle$. By (c), $q$ is a condition in $\mathbf{R}_{\kappa}$.

Let $r \in A$ be compatible with $q$ and let $r^{*} \in \mathbf{R}_{\kappa}$ be such that $r^{*} \leqslant r \wedge q$. Define a new condition $p^{*} \in \mathbf{P}_{\lambda}$ as follows:

Let $p^{*} \mid \psi(0)$ be any condition in $\mathbf{P}_{\psi(0)}$ such that $p^{*}\left|\psi(0) \leqslant \varphi_{0}\left(r^{*}(0)\right) \wedge p\right| \psi(0)$. Such a condition exists since $r^{*}(0) \leqslant \pi_{0}(p \nmid \psi(0))$. For $\beta \geqslant \psi(0)$, let

$$
p^{*}(\beta)= \begin{cases}p(\beta) & \text { if } \beta \notin \psi^{\prime \prime} K, \\ r^{*}(\alpha) & \text { if } \beta=\psi(\alpha),\end{cases}
$$

$p^{*}$ is a condition since it has support

$$
\left(\operatorname{supp} p^{*}\lceil\psi(0)) \cup \operatorname{supp} p .\right.
$$

It is now easy to verify by induction that $p^{*} \leqslant \varphi\left(r^{*}\right) \wedge p$, contradicting $p$ being incompatible with all of $\varphi^{\prime \prime} A$.

One verifies similarly that if $r_{1}$ and $r_{2}$ are incompatible then so are $\varphi\left(r_{1}\right)$ and $\varphi\left(r_{2}\right)$.

In practice this lemma will be used for inductively defining neat embeddings between iterations as they are built. We will be defining two iterations $\mathbf{P}$ and $\mathbf{R}$ and step by step extending the maps from $\mathbf{R}$ into $\mathbf{P}$ using Lemma 5.1 at limit stages.

6. We now describe our partial orders. For all pairs of Mahlo cardinals $\lambda, \kappa$ with $\lambda<\kappa$ and every $\lambda$ c.c. $\mathbf{Q}$ included in $V_{\lambda}$, we will define a partial ordering $\mathbf{Q} * \mathbf{R}(\lambda, \kappa, X,<)$. The parameter $X$ will be a set of ordered pairs $\left\langle\mathbf{Q}^{\prime}, e^{\prime}\right\rangle$ such that $e^{\prime}: \mathbf{Q}^{\prime} \hookrightarrow \mathbf{Q}$, and $<$ will be a well ordering of $X$. Our final partial order will be

$$
\mathbf{P}\left(\kappa_{0}\right) * \mathbf{R}\left(\kappa_{0}, \kappa_{1}, \Gamma_{\kappa_{0}},<\right) * \bar{S}\left(\kappa_{1}, \kappa_{2}\right)
$$

for $\Gamma_{\kappa_{0}}$ and $<$ to be defined later. One of the crucial properties we will be trying to ensure is that

$$
\left\langle\mathbf{P}\left(\kappa_{0}\right) * \mathbf{R}\left(\kappa_{0}, \kappa_{1}, \Gamma_{\kappa_{0}},<\right), e^{\prime}\right\rangle \in j\left(\Gamma_{\kappa_{0}}\right)
$$

for some canonically constructed $e^{\prime}$. 
We will go by induction on $\lambda$ and $\kappa$. Suppose for all $\lambda^{\prime}, \kappa^{\prime}<\lambda, X_{\mathbf{Q}^{\prime}}$ and $\mathbf{Q}^{\prime} * \mathbf{R}\left(\lambda^{\prime}, \kappa^{\prime}, X,<\right)$ have been defined for all $\mathbf{Q}^{\prime} \subseteq V_{\lambda^{\prime}}$ with $\mathbf{Q}^{\prime} \lambda^{\prime}$ c.c., all $X \subseteq X_{\mathbf{Q}^{\prime}}$, and $<$ a well ordering of $X$.

Let $\mathbf{Q}$ be a $\lambda$ c.c. partial ordering with $Q \subseteq V_{\lambda}$. Let $X_{\mathbf{Q}}=\left\{\left\langle\mathbf{Q}^{\prime}, e\right\rangle \mid e: \mathbf{Q}^{\prime} \hookrightarrow \mathbf{Q}\right.$ and $\mathbf{Q}^{\prime}=\mathbf{P}^{\prime} * \mathbf{R}\left(\lambda^{\prime}, \lambda^{\prime \prime}, X^{\prime},<^{\prime}\right)$ for some $\left.\mathbf{P}^{\prime} \subseteq V_{\lambda^{\prime}}, X^{\prime} \subseteq X_{\mathbf{P}^{\prime}}\right\} \cup\{\langle\mathbf{Q}$, id $\rangle\}$. ( $X_{\mathbf{Q}}$ really depends on $\mathbf{Q}$ and $\lambda$ but in context $\lambda$ will be clear, so we drop it to reduce notational complexity.)

$\mathbf{Q} * \mathbf{R}(\lambda, \kappa, X,<)$ will be constructed in two phases. In the first phase we will be taking steps so that when we are done

$$
\mathbf{P}\left(\kappa_{0}\right) * \mathbf{R}\left(\kappa_{0}, \kappa_{1}, \Gamma_{\kappa_{0}},<_{\kappa_{0}, \kappa_{1}}\right) * \bar{S}\left(\kappa_{1}, \kappa_{2}\right) \hookrightarrow j\left(\mathbf{P}\left(\kappa_{0}\right) * \mathbf{R}\left(\kappa_{0}, \kappa_{1}, \Gamma_{\kappa_{0}},<_{\kappa_{0}, \kappa_{1}}\right)\right) .
$$

In the second phase we will make sure that when we are done, for almost all $\alpha<\kappa_{0}$ there is an $i$ such that the following diagram commutes:

$$
\begin{array}{rl}
\mathbf{P}(\alpha) * & \mathbf{R}\left(\alpha, \kappa_{0}\right) \\
& \oint_{j} \\
\mathbf{P}(\alpha) * \mathbf{R}\left(\alpha, \kappa_{1}\right)
\end{array} \quad \mathbf{P}(\alpha) * \mathbf{R}\left(\alpha, \kappa_{0}\right) * \bar{S}\left(\kappa_{0}, \kappa_{1}\right)
$$

The first phase will be an iteration with supports of cardinality $<\lambda$, the second phase will use cardinality $\lambda$ support.

Let $\kappa>\lambda, \kappa$ Mahlo. We assume that $\mathbf{Q} * \mathbf{R}\left(\lambda, \kappa^{\prime}, X,<\right)$ has been defined for all $X \subseteq X_{\mathbf{Q}}, \kappa^{\prime}<\kappa$ and $<$ a well ordering of $X$.

Let $X \subseteq X_{\mathbf{Q}},<$ a well ordering of $X$ and $\gamma=$ o.t. $<$. The first phase of the construction will be $\mathbf{Q} * \mathbf{R}_{\gamma}(\lambda, \kappa, X,<)$.

Phase 1. Let $\mathbf{Q} * \mathbf{R}_{0}(\lambda, \kappa, X,<)=\mathbf{Q}$. At stage $\alpha+1$ : Let $\left\langle\mathbf{Q}_{\alpha}, e_{\alpha}\right\rangle$ be the $\alpha$ th member of $X$, then

$$
\mathbf{Q} * \mathbf{R}_{\alpha+1}(\lambda, \kappa, X,<)=\mathbf{Q} * \mathbf{R}_{\alpha}(\lambda, \kappa, X,<) *_{e_{\alpha}} S^{\mathbf{Q}_{\alpha}}(\lambda, \kappa) .
$$

$\left\langle\mathbf{Q}_{\alpha}, e_{\alpha}\right\rangle$ is said to be handled at stage $\alpha$.

For $\alpha$ a limit: We take the iteration with support $\mathcal{K}_{\alpha}$ where $\mathcal{K}_{\alpha}=\{x \subseteq \alpha$ : $|x|<\lambda\}$.

If $\boldsymbol{\kappa}$ is the first Mahlo cardinal beyond $\lambda$ then the construction stops here.

Phase 2. Enumerate the Mahlo cardinals less than $\kappa,\left\langle\lambda_{\alpha}: \alpha<\kappa\right\rangle$ in a canonical well ordering so that each appears $\kappa$ times. At stage $\alpha+1$ :

Case a. id: $\mathbf{Q} * \mathbf{R}\left(\lambda, \lambda_{\alpha}, X,<\right) \hookrightarrow \mathbf{Q} * \mathbf{R}_{\alpha}(\lambda, \kappa, X,<), \lambda_{\alpha}$ has not been handled yet and $\alpha>\lambda_{\alpha}$.

Let

$$
\mathbf{Q} * \mathbf{R}_{\alpha+1}(\lambda, \kappa, X,<)=\mathbf{Q} * \mathbf{R}_{\alpha}(\lambda, \kappa, X,<){ }_{\text {id }} S^{\mathbf{Q} * \mathbf{R}\left(\lambda, \lambda_{\alpha}, X,<\right)}\left(\lambda_{\alpha}, \kappa\right)
$$

$\lambda_{\alpha}$ is said to have been handled at stage $\alpha$.

Case b. Otherwise. Let

$$
\mathbf{Q} * \mathbf{R}_{\alpha+1}(\lambda, \kappa, X,<)=\mathbf{Q} * \mathbf{R}_{\alpha}(\lambda, \kappa, X,<) * 2
$$


At limit stages $\alpha$ :

$\mathbf{Q} * \mathbf{R}_{\alpha}(\lambda, \kappa, X,<)$ is the iteration with support $\mathscr{K}_{\alpha}=\{x:|x \cap \gamma|<\lambda$ and $|x| \leqslant$ $\lambda\}$.

It is worth noting that this definition of $\mathscr{K}$ corresponds to the direct limit at inaccessible $\alpha>\lambda$. The construction terminates at stage $\kappa$.

From time to time we will abuse notation and write " $\langle q, s\rangle \in \mathbf{Q} * \mathbf{R}(\lambda, \kappa, X,<)$ with $q \in \mathbf{Q}, s \in \mathbf{R}(\lambda, \kappa, X,<)$ " when what is really meant is the function $\sigma \in$ $\mathbf{Q} * \mathbf{R}(\lambda, \kappa, X,<)$ defined by

$$
\sigma(\alpha)=\left\{\begin{array}{l}
q \text { if } \alpha=0 \\
s(\alpha) \text { if } \alpha \neq 0 .
\end{array}\right.
$$

It is now easy to see that if $\left\langle\mathbf{Q}^{\prime}, e\right\rangle \in X$ then

$$
\mathbf{Q}^{\prime} * S^{\mathbf{Q}^{\prime}}(\lambda, \kappa) \underset{e * i}{\hookrightarrow} \mathbf{Q} * \mathbf{R}(\lambda, \kappa, X,<)
$$

for some $i$. This is because $\left\langle\mathbf{Q}^{\prime}, e\right\rangle$ will appear as some $\left\langle\mathbf{Q}_{\alpha}, e_{\alpha}\right\rangle$, so

$$
\mathbf{Q}^{\prime} * S^{\mathbf{Q}^{\prime}}(\lambda, \kappa) \hookrightarrow \mathbf{Q} * \mathbf{R}_{\alpha+1}(\lambda, \kappa, X,<)
$$

by Lemma 2.3. Further, if $\kappa^{\prime}<\kappa$ and $\mathbf{Q} * \mathbf{R}\left(\lambda, \kappa^{\prime}, X,<\right) \hookrightarrow \mathbf{Q} * \mathbf{R}(\lambda, \kappa, X,<)$ then

$$
\mathbf{Q} * \mathbf{R}\left(\lambda, \kappa^{\prime}, X,<\right) * \bar{S}\left(\kappa^{\prime}, \kappa\right) \underset{\text { id } * i}{\hookrightarrow} \mathbf{Q} * \mathbf{R}(\lambda, \kappa, X,<)
$$

for some $i$. We know this because $\mathbf{Q} * \mathbf{R}\left(\lambda, \boldsymbol{\kappa}^{\prime}, X,<\right)$ is an iteration of length $\boldsymbol{\kappa}^{\prime}$, thus the image of id on $\mathbf{Q} * \mathbf{R}\left(\lambda, \boldsymbol{\kappa}^{\prime}, X,<\right)$ is contained in $\mathbf{Q} * \mathbf{R}_{\boldsymbol{\kappa}^{\prime}+1}(\lambda, \kappa, X,<)$. Since $\boldsymbol{\kappa}^{\prime}$ will be handled at some stage $\alpha>\kappa^{\prime}$,

$$
\mathbf{Q} * \mathbf{R}\left(\lambda, \kappa^{\prime}, X,<\right) * \bar{S}\left(\kappa^{\prime}, \kappa\right) \hookrightarrow \mathbf{Q} * \mathbf{R}_{\alpha+1}(\lambda, \kappa, X,<)
$$

We can check by induction that $\mathbf{Q} * \mathbf{R}(\lambda, \kappa, X,<) \subseteq V_{\kappa}$, using the fact that $\left|X_{\mathbf{Q}}\right| \leqslant 2^{\lambda}$ for $|\mathbf{Q}|=\lambda$. (We view elements of $\mathbf{Q} * \mathbf{R}(\lambda, \kappa, X,<$ ) as functions with domain the support of the condition as mentioned in $§ 5$.)

We will now describe the partial order $\mathbf{P}\left(\kappa_{0}\right)$. We will define $\mathbf{P}(\lambda)$ for all Mahlo $\lambda$. As we build $\mathbf{P}(\lambda)$ we will want that if $\mathbf{P}(\alpha) \hookrightarrow \mathbf{P}(\lambda)$ then there is a canonical map icon $^{(\alpha, \lambda)}$ such that the following commutes:

$$
\begin{gathered}
\mathbf{P}(\alpha) \underset{\operatorname{id}}{\rightarrow} \mathbf{P}(\lambda) \\
\operatorname{id} \uparrow \quad \operatorname{licon}^{(\alpha, \lambda)} \\
\mathbf{P}(\alpha) * \mathbf{R}\left(\alpha, \lambda_{\alpha}, \Gamma_{\alpha},<_{\alpha, \lambda}\right) .
\end{gathered}
$$

$\Gamma_{\alpha} \subseteq X_{\mathbf{P}(\alpha)}$ and $<_{\alpha, \lambda}$ will be defined inductively. $\Gamma_{\alpha}$ will be defined as soon as $\mathbf{P}(\alpha)$ is, but the definition of $<_{\alpha, \lambda}$ occurs during the construction of $\mathbf{P}(\lambda)$.

Let $\lambda$ be a Mahlo cardinal. Assume as an induction hypothesis that for all $\alpha, \beta$ Mahlo, $\alpha<\beta<\lambda$, the following have been defined:

(a) $\mathbf{P}(\alpha)$,

(b) $\Gamma_{\beta}$,

(c) $<_{\alpha, \beta}$,

(d) $\operatorname{icon}^{(\alpha, \beta)}$, with

$$
\Gamma_{\beta}=\left\{\left\langle\mathbf{P}(\alpha) * \mathbf{R}\left(\alpha, \beta, \Gamma_{\alpha},<_{\alpha, \beta}\right), \operatorname{icon}^{(\alpha, \beta)}\right\rangle \mid \mathbf{P}(\alpha) \underset{\text { id }}{\hookrightarrow} \mathbf{P}(\beta)\right\} \cup\{\langle\mathbf{P}(\beta), \text { id }\rangle\} .
$$


We will now proceed in defining $\mathbf{P}(\lambda),<_{\alpha, \lambda}$ and $\operatorname{icon}^{(\alpha, \lambda)}$. The map icon ${ }^{(\alpha, \lambda)}$ will be defined using the technique of $\$ 5$.

During the construction we will be defining several notions by induction and making various promises about the construction which must be kept:

(1) For every partial order $\mathbf{P}(\alpha)$ we consider we will define the notion of a $\langle\mathbf{P}(\alpha), 0\rangle$ coordinate and a $\langle\mathbf{P}(\alpha), 1\rangle$ coordinate. These will be the coordinates of $\mathbf{P}(\lambda)$ in the image of the map

$$
\operatorname{icon}^{(\alpha, \lambda)}: \mathbf{P}(\alpha) * \mathbf{R}\left(\alpha, \lambda, \Gamma_{\alpha},<_{\alpha, \lambda}\right) \hookrightarrow \mathbf{P}(\lambda)
$$

$\langle\mathbf{P}(\alpha), 0\rangle$ coordinates will correspond to the first phase of the construction of $\mathbf{P}(\alpha) * \mathbf{R}(\alpha, \lambda, X,<) .\langle\mathbf{P}(\alpha), 1\rangle$ coordinates will correspond to the second phase of the construction.

(2) Type $\gamma$ coordinates.

Type $\gamma,\langle\mathbf{P}(\boldsymbol{\alpha}), 0\rangle$ and $\langle\mathbf{P}(\boldsymbol{\alpha}), 1\rangle$ coordinates are a labeling system designed to facilitate the use of Lemma 5.1. At a stage $\delta$ in the construction of $\mathbf{P}(\lambda)$ we will decide what $\mathbf{P}_{\delta+1}(\lambda)$ is and decide whether to label $\delta$ a $\langle\mathbf{P}(\alpha), 0\rangle,\langle\mathbf{P}(\alpha), 1\rangle$ or a type $\gamma$ coordinate. Once a label is applied to $\delta$ it never loses the label. Every type $\gamma$ and $\langle\mathbf{P}(\alpha), 1\rangle$ coordinate $\delta$ will receive that label at stage $\delta$ in the construction, however, some $\langle\mathbf{P}(\gamma), 0\rangle$ coordinate $\delta$ will have that label applied after stage $\delta$. A coordinate can have several labels, e.g., all $\langle\mathbf{P}(\gamma), 0\rangle$ coordinates will be type $\gamma$. No $\langle\mathbf{P}(\gamma), 1\rangle$ coordinate will be type $\gamma$.

In addition we will be making five promises, P1-P5, which can be viewed as induction hypotheses. The first three are:

P1. If $\alpha \neq \alpha^{\prime}$,

$$
\{\gamma: \gamma \text { is of type }\langle\mathbf{P}(\alpha), 1\rangle\} \cap\left\{\gamma: \gamma \text { is of type }\left\langle\mathbf{P}\left(\alpha^{\prime}\right), 1\right\rangle\right\}=\varnothing .
$$

P2. For all $\alpha$ and $\mu$ with $\alpha \neq \mu$ there is at most one $\delta$ that is both type $\mu$ and $\langle\mathbf{P}(\alpha), 1\rangle$.

P3. For $\gamma \neq \gamma^{\prime}$, type $\gamma \cap$ type $\gamma^{\prime}=\varnothing$.

(3) The notion of being under attack. To begin with no partial ordering will be under attack. As we proceed, we will consider partial orderings $\mathbf{P}(\alpha)$. When the time is right and certain conditions are satisfied we will attack $\mathbf{P}(\alpha)$ by beginning to iterate $\mathbf{P}(\alpha) * \mathbf{R}\left(\alpha, \lambda, \Gamma_{\alpha},<_{\alpha, \lambda}\right)$ stage by stage into $\mathbf{P}(\lambda)$ using the technique of $\S 5$.

P4. We promise never to attack $\mathbf{P}(\alpha)$ before stage $\alpha$ in the construction of $\mathbf{P}(\lambda)$.

(4) For each partial ordering $\mathbf{P}(\alpha)$ under attack we will define a well ordering $<_{\alpha, \lambda}$ of $\Gamma_{\alpha}$. This definition will occur at the moment $\mathbf{P}(\alpha)$ comes under attack.

(5) At each stage $\delta$ we will be building a map

$$
\operatorname{icon}_{\beta}^{(\alpha, \lambda)}: \mathbf{P}(\alpha) * \mathbf{R}_{\beta}\left(\alpha, \lambda, \Gamma_{\alpha},<_{\alpha, \lambda}\right) \hookrightarrow \mathbf{P}_{\delta}(\lambda)
$$

stage by stage using $\$ 5$ techniques.

(6) The notion of being "handled". If $\mathbf{P}(\alpha)$ is under attack and

$$
\mathbf{P}(\alpha) * \mathbf{R}_{\beta+1}\left(\alpha, \lambda, \Gamma_{\alpha},<_{\alpha, \lambda}\right)=\mathbf{P}(\alpha) * \mathbf{R}_{\beta}\left(\alpha, \lambda, \Gamma_{\alpha},<_{\alpha, \lambda}\right){ }_{e} S^{\mathbf{Q}}(\eta, \lambda),
$$

then at some stage $\gamma \geqslant \beta$ we will emulate this by

$$
\mathbf{P}_{\gamma+1}(\lambda)=\mathbf{P}_{\gamma}(\lambda) *_{\text {icon }_{\beta}^{(\alpha, \lambda)} \circ e} S^{\mathbf{Q}}(\eta, \lambda) .
$$


By Lemma 2.4, we can extend icon ${ }_{\beta}^{(\alpha, \lambda)}$ to

$$
\operatorname{icon}_{\beta+1}^{(\alpha, \lambda)}: \mathbf{P}(\alpha) * \mathbf{R}_{\beta+1}\left(\alpha, \lambda, \Gamma_{\alpha},<_{\alpha, \lambda}\right) \hookrightarrow \mathbf{P}_{\gamma+1}(\lambda) .
$$

$\langle\alpha, \beta\rangle$ will be said to have been "handled" at stage $\gamma$.

P5. (a) If $p \in \mathbf{P}(\alpha) * \mathbf{R}_{\beta}\left(\alpha, \lambda, \Gamma_{\alpha},<_{\alpha, \lambda}\right)$ then

$$
\begin{aligned}
\operatorname{suppicon}_{\beta}^{(\alpha, \lambda)}(p) \subseteq & \operatorname{suppicon}_{\beta}^{(\alpha, \lambda)}(p(0)) \cup \operatorname{type}(\mathbf{P}(\alpha), 0) \\
& \cup \operatorname{type}(\mathbf{P}(\alpha), 1) .
\end{aligned}
$$

(b) If $p \in \mathbf{P}(\alpha) * \mathbf{R}_{\beta}\left(\alpha, \lambda, \Gamma_{\alpha},<_{\alpha, \lambda}\right)$ and $p$ is in the first phase of $\mathbf{P}(\alpha) * \mathbf{R}_{\beta}\left(\alpha, \lambda, \Gamma_{\alpha},<_{\alpha, \lambda}\right)$ then

$$
\operatorname{suppicon}_{\beta}^{(\alpha, \lambda)}(p) \subseteq \operatorname{suppicon}_{\beta}^{(\alpha, \lambda)}(p(0)) \cup \operatorname{type}(\mathbf{P}(\alpha), 0) .
$$

(c) For each $\alpha$ under attack, we let $\psi(0)=\alpha, \psi(\beta)=$ the stage where $\langle\alpha, \beta\rangle$ is handled for $\beta>0$. If $\beta^{\prime}$ is in the first phase of $\mathbf{P}(\alpha) * \mathbf{R}_{\beta}\left(\alpha, \lambda, \Gamma_{\alpha},<_{\alpha, \lambda}\right)$ then $\psi\left(\beta^{\prime}\right)$ is of the type $(\mathbf{P}(\alpha), 0)$. If $\beta^{\prime}$ is in the second phase of the construction of $\mathbf{P}(\alpha) * \mathbf{R}_{\beta}\left(\alpha, \lambda, \Gamma_{\alpha},<_{\alpha, \lambda}\right), \psi\left(\beta^{\prime}\right)$ is a type $(\mathbf{P}(\alpha), 1)$ coordinate.

$\mathbf{P}(\lambda)$ will be a $\lambda$-stage iteration. We will take direct limits at all inaccessibles. At limit stages $\gamma$, where $\gamma$ is not inaccessible, we will take all conditions $p \in \lim _{\beta<\gamma} \mathbf{P}_{\beta}(\lambda)$ such that:

(1) For any $\mathbf{P}(\mu)$ under attack:

$$
\begin{aligned}
& \text { (a) }|\operatorname{supp} p \cap \operatorname{type}(\mathbf{P}(\mu), 0)|<\mu \text {, } \\
& \text { (b) }|\operatorname{supp} p \cap \operatorname{type}(\mathbf{P}(\mu), 1)| \leqslant \mu \text {. }
\end{aligned}
$$

(2) For any $\gamma$ Mahlo, $\mid \operatorname{supp} p \cap$ type $\gamma \mid<\gamma$ (i.e., we will take the iteration with support $\mathscr{K}_{\xi}=\left\{x \subseteq \xi\right.$ : for all $\alpha<\xi x \cap \alpha \in \mathscr{K}_{\alpha}$ and $x$ satisfies (1) and (2) above $\}$ ).

At successors of inaccessibles $\gamma$, we will consider partial orderings $\mathbf{P}(\alpha)$ not yet under attack. If $\mathbf{P}(\alpha) \hookrightarrow \mathbf{P}_{\gamma}(\lambda)$ and certain other conditions are satisfied then we will begin iterating in $\mathbf{P}(\alpha) * \mathbf{R}\left(\alpha, \lambda, \Gamma_{\alpha},<_{\alpha, \lambda}\right)$. At this stage we will define $<_{\alpha, \lambda}$.

To make the notation less cumbersome, as soon as $\Gamma_{\alpha},<_{\alpha, \lambda}$ have been defined, we will drop them from the parameters in the R's; i.e., $\mathbf{P}(\alpha) * \mathbf{R}(\alpha, \lambda)$ instead of $\mathbf{P}(\alpha) * \mathbf{R}\left(\alpha, \lambda, \Gamma_{\alpha},<_{\alpha, \lambda}\right)$.

To start off, if $\lambda$ is the least Mahlo, $\mathbf{P}(\lambda)=S(\omega, \lambda)$. Otherwise, canonically enumerate $\{\mathbf{P}(\alpha): \alpha<\lambda\}$ as $\left\langle\mathbf{P}\left(\alpha_{\beta}\right): \beta<\lambda\right\rangle$ so that each $\mathbf{P}(\alpha)$ appears $\lambda$ many times. We well order the pairs $\langle\alpha, \beta\rangle$ as follows:

$$
\langle\alpha, \beta\rangle\left\langle\left\langle\alpha^{\prime}, \beta^{\prime}\right\rangle\right. \text { iff }
$$

(1) $\max (\alpha, \beta)<\max \left(\alpha^{\prime}, \beta^{\prime}\right)$ or $\max (\alpha, \beta)=\max \left(\alpha^{\prime}, \beta^{\prime}\right)$ and

(2) $\alpha<\alpha^{\prime}$ or $\alpha=\alpha^{\prime}$ and

(3) $\beta<\beta^{\prime}$.

We will maintain an induction hypothesis:

H. If $\gamma$ is the least stage where $\mathbf{P}_{\gamma+1}$ is not defined, $\mathbf{P}(\alpha)$ is under attack and $\beta$ is the least ordinal such that $\langle\alpha, \beta\rangle$ has not been handled, then $\operatorname{icon}_{\beta}^{(\alpha, \beta)}$ : $\mathbf{P}(\alpha) * \mathbf{R}_{\beta}(\alpha, \lambda) \hookrightarrow \mathbf{P}_{\gamma}(\lambda)$. 
Further, if $\psi: \beta \rightarrow \gamma$ is defined by: $\psi(\eta)=$ the stage where $\langle\alpha, \eta\rangle$ has been handled, then $\operatorname{icon}_{\beta}^{(\alpha, \lambda)}$ is the map $\varphi$ given by Lemma 5.1 from $\mathbf{P}(\alpha) * \mathbf{R}_{\beta}(\alpha, \lambda) \hookrightarrow$ $\mathbf{P}_{\gamma}(\lambda)$.

(We will consider a map icon $\operatorname{ic}_{\beta}^{(\alpha, \lambda)}: \mathbf{P}(\alpha) * \mathbf{R}_{\beta}(\alpha, \lambda) \hookrightarrow \mathbf{P}_{\xi}(\lambda)$ for $\xi<\gamma$ as having range in $\mathbf{P}_{\gamma}(\lambda)$ by identifying it with id $\circ \operatorname{icon}(\alpha, \lambda)$ where id: $\mathbf{P}_{\xi}(\lambda) \hookrightarrow \mathbf{P}_{\gamma}(\lambda)$.)

To start, let $\mathbf{P}_{0}(\lambda)=S(\omega, \lambda)$.

Case a. $\gamma+1$ is the least $\xi$ such that $\mathbf{P}_{\xi}(\lambda)$ is not defined and $\gamma$ is not inaccessible.

Let $\langle\alpha, \beta\rangle$ be the least pair such that $\mathbf{P}(\alpha)$ is under attack and $\langle\alpha, \beta\rangle$ has not been handled yet. (If no such pair exists then $\mathbf{P}_{\gamma+1}(\lambda)=\mathbf{P}_{\gamma}(\lambda) * 2$.)

Then, either $\mathbf{P}(\alpha) * \mathbf{R}_{\beta+1}(\alpha, \lambda)=\mathbf{P}(\alpha) * \mathbf{R}_{\beta}(\alpha, \lambda) * 2$, in which case we let $\mathbf{P}_{\gamma+1}(\lambda)=\mathbf{P}_{\gamma}(\lambda) * 2$, or

$$
\mathbf{P}(\alpha) * \mathbf{R}_{\beta+1}(\alpha, \lambda)=\mathbf{P}(\alpha) * \mathbf{R}_{\beta}(\alpha, \lambda) *_{\text {id }} S^{\mathbf{Q}}\left(\lambda_{\beta}, \lambda\right)
$$

for some $\mathbf{Q}=\mathbf{P}(\alpha) * \mathbf{R}\left(\alpha, \lambda_{\beta}\right)$. If the latter, we let

$$
\mathbf{P}_{\gamma+1}(\lambda)=\mathbf{P}_{\gamma}(\lambda) *_{\text {icon }_{\beta}^{(\alpha, \lambda) \circ \text { id }}} S^{\mathbf{Q}}\left(\lambda_{\beta}, \lambda\right) .
$$

Let $\operatorname{icon}_{\beta+1}^{(\alpha, \lambda)}: \mathbf{P}(\alpha) * \mathbf{R}_{\beta+1}(\alpha, \lambda) \rightarrow \mathbf{P}_{\gamma+1}(\lambda)$ be the embedding guaranteed by Lemma 2.3. We say:

(0) $\mathbf{P}(\alpha) * \mathbf{R}\left(\alpha, \lambda_{\beta}\right)$ has been handled at this stage, $\gamma+1$.

(1) $\langle\alpha, \beta\rangle$ has been handled at stage $\gamma+1$.

(2) $\gamma$ is called a $\langle\mathbf{P}(\alpha), 1\rangle$ coordinate.

(3) In the second alternative above, we call $\gamma$ a type $\lambda_{\beta}$ coordinate.

Case b. $\gamma$ is the least place, $\mathbf{P}_{\gamma}(\lambda)$ is not defined and $\gamma$ is a limit.

(i) If $\gamma$ is inaccessible then $\mathbf{P}_{\gamma}(\lambda)=\lim \mathbf{P}_{\alpha}(\lambda)$.

(ii) If $\gamma$ is not inaccessible, then we take all conditions $p \in \lim \mathbf{P}_{\gamma}(\lambda)$ such that:

(1) for all $\mathbf{P}(\mu)$ under attack

$$
\begin{aligned}
& |\operatorname{supp} p \cap \operatorname{type}\langle\mathbf{P}(\mu), 0\rangle|<\mu, \\
& |\operatorname{supp} p \cap \operatorname{type}\langle\mathbf{P}(\mu), 1\rangle| \leqslant \mu .
\end{aligned}
$$

(2) For any inaccessible $\mu, \mid \operatorname{supp} p \cap$ type $\mu \mid<\mu$.

We have to verify our induction hypothesis $H$ at this stage. We use Lemma 5.1.

Consider an $\alpha$ such that $\mathbf{P}(\alpha)$ is under attack. Let $\beta$ be the least ordinal such that $\langle\alpha, \beta\rangle$ has not been handled. By $\mathrm{P} 4, \alpha<\gamma$.

If $\eta=\sup \left\{\delta: \delta\right.$ is the stage where some $\left\langle\alpha, \beta^{\prime}\right\rangle$ has been handled for some $\left.\beta^{\prime}<\beta\right\}<\gamma$, then, by $\mathrm{H}$ we have that

$$
\operatorname{icon}_{\beta}^{(\alpha, \lambda)}: \mathbf{P}(\alpha) * \mathbf{R}_{\beta}(\alpha, \lambda) \hookrightarrow \mathbf{P}_{\eta}(\lambda) .
$$

Since id: $\mathbf{P}_{\eta}(\lambda) \rightarrow \mathbf{P}_{\gamma}(\lambda)$, H holds at $\gamma$.

If $\eta=\gamma$, we let $\psi(0)=\alpha$ and $\psi\left(\beta^{\prime}\right)=$ the stage where $\left\langle\alpha, \beta^{\prime}\right\rangle$ is handled. We want to verify the hypothesis of Lemma 5.1.

Case i. $\gamma$ is inaccessible.

The observation above yields that $\beta=\gamma$. Since $\alpha<\gamma$,

$$
\mathbf{P}(\alpha) * \mathbf{R}_{\beta}(\alpha, \lambda)=\lim _{\xi<\beta} \mathbf{P}(\alpha) * \mathbf{R}_{\xi}(\alpha, \lambda) .
$$


Thus we are taking direct limits in both $\mathbf{P}(\alpha) * \mathbf{R}_{\beta}(\alpha, \lambda)$ and $\mathbf{P}_{\gamma}(\lambda)$. Consequently, the support criterion of Lemma 5.1 is easy to establish. Applying the lemma, we get a canonical

$$
\operatorname{icon}_{\beta}^{(\alpha, \lambda)}: \mathbf{P}(\alpha) * \mathbf{R}_{\beta}(\alpha, \lambda) \hookrightarrow \mathbf{P}_{\gamma}(\lambda) .
$$

Case ii. $\gamma$ is not inaccessible.

Let $X$ be the support of a condition $p \in \mathbf{P}(\alpha) * \mathbf{R}_{\beta}(\alpha, \lambda)$. We need that $\psi^{\prime \prime} X$ is a support for $\mathbf{P}_{\gamma}(\lambda)$. By P5, $\left|\psi^{\prime \prime} X \cap \operatorname{type}(\mathbf{P}(\alpha), 0)\right|<\alpha$, because $X$ restricted to the first phase of $\mathbf{P}(\alpha) * \mathbf{R}_{\beta}(\alpha, \lambda)$ has cardinality $<\alpha$. Since $|X| \leqslant \alpha, \quad \mid \psi^{\prime \prime} X \cap$ type $(\mathbf{P}(\alpha), 1) \mid \leqslant \alpha$. Now $\psi^{\prime \prime} X \subseteq \operatorname{type}(\mathbf{P}(\alpha), 0) \cup$ type $(\mathbf{P}(\alpha), 1)$, and for any $\mu \neq \alpha$,

$$
\mid \text { type } \mu \cap(\operatorname{type}(\mathbf{P}(\alpha), 0) \cup \operatorname{type}(\mathbf{P}(\alpha), 1)) \mid \leqslant 1,
$$

so $\mid \psi^{\prime \prime} X \cap$ type $\mu \mid<\mu$. If $\mu=\alpha$, then type $\mu \cap \psi^{\prime \prime} X \subseteq \operatorname{type}(\mathbf{P}(\alpha), 0)$ so $\mid \psi^{\prime \prime} X \cap$ type $\alpha \mid<\alpha$. Similarly, P1 and P2 imply that for any $\mu \neq \alpha$,

$$
\begin{aligned}
& \left|\psi^{\prime \prime} X \cap \operatorname{type}(\mathbf{P}(\mu), 0)\right| \leqslant 1, \\
& \left|\psi^{\prime \prime} X \cap \operatorname{type}(\mathbf{P}(\mu), 1)\right| \leqslant 1 .
\end{aligned}
$$

Thus $\psi^{\prime \prime} X$ is a support for $\mathbf{P}_{\gamma}(\lambda)$.

Let $X \subseteq \beta$ be such that $\psi^{\prime \prime} X$ is the support for some condition in $\mathbf{P}_{\gamma}(\lambda)$. Then, $\left|\psi^{\prime \prime} X \cap \operatorname{type}(\mathbf{P}(\alpha), 0)\right|<\alpha$, so $X$ restricted to the first phase of $\mathbf{P}(\alpha) * \mathbf{R}_{\beta}(\alpha, \lambda)$ has cardinality $<\alpha$. Since $\psi^{\prime \prime} X \subseteq \operatorname{type}(\mathbf{P}(\alpha), 0) \cup \operatorname{type}(\mathbf{P}(\alpha), 1),\left|\psi^{\prime \prime} X\right| \leqslant \alpha$. Thus $|X| \leqslant$ $\alpha$ and $X$ is a support for $\mathbf{P}(\alpha) * \mathbf{R}_{\beta}(\alpha, \lambda)$. This establishes the support criterion in Lemma 5.1. Applying the lemma we get a map

$$
\operatorname{icon}_{\beta}^{(\alpha, \lambda)}: \mathbf{P}(\alpha) * \mathbf{R}_{\beta}(\alpha, \lambda) \hookrightarrow \mathbf{P}_{\gamma}(\lambda)
$$

satisfying the induction hypothesis.

Case c. $\gamma+1$ is the least ordinal such that $\mathbf{P}_{\gamma+1}(\lambda)$ is not defined and $\gamma$ is inaccessible.

Suppose $\gamma$ is the $\beta$ th inaccessible. We consider the $\beta$ th $\mathbf{P}(\alpha)$ in our enumeration. If it is not yet under attack, we ask:

(1) id: $\mathbf{P}(\alpha) \hookrightarrow \mathbf{P}_{\gamma}(\lambda)$ ?

(2) $\alpha<\gamma$ ?

If the answer to either one of these questions is no, then $\mathbf{P}_{\gamma+1}(\lambda)=\mathbf{P}_{\gamma}(\lambda) * 2$ and $\mathbf{P}(\alpha)$ is still not under attack. If yes:

Let $\Gamma_{\gamma}^{\prime} \subseteq \Gamma_{\alpha}$ be defined by: $\mathbf{P}\left(\alpha^{\prime}\right) * \mathbf{R}\left(\alpha^{\prime}, \alpha\right) \in \Gamma_{\gamma}^{\prime}$ iff

(1) $\alpha^{\prime}<\alpha$,

(2) $\mathbf{P}\left(\alpha^{\prime}\right)$ is under attack,

(3) $<_{\alpha^{\prime}, \alpha}=<_{\alpha^{\prime}, \lambda}$,

(4) id: $\mathbf{P}\left(\alpha^{\prime}\right) * \mathbf{R}\left(\alpha^{\prime}, \alpha\right) \hookrightarrow \mathbf{P}\left(\alpha^{\prime}\right) * \mathbf{R}_{\delta}\left(\alpha^{\prime}, \lambda\right)$,

where $\delta$ is the greatest ordinal such that

$$
\operatorname{icon}_{\delta}^{\left(\alpha^{\prime}, \lambda\right)}: \mathbf{P}\left(\alpha^{\prime}\right) * \mathbf{R}_{\delta}\left(\alpha^{\prime}, \lambda\right) \hookrightarrow \mathbf{P}_{\gamma}(\lambda)
$$

is defined. 
(5) The diagram

$$
\begin{aligned}
& \mathbf{P}\left(\alpha^{\prime}\right) * \mathbf{R}\left(\alpha^{\prime}, \alpha\right) \quad \stackrel{\text { id }}{\mathbf{P}}\left(\alpha^{\prime}\right) * \mathbf{R}_{\delta}\left(\alpha^{\prime}, \lambda\right) \\
& \int_{\text {icon }}{ }^{\left(\alpha^{\prime}, \alpha\right)} \quad \int_{\text {icon }}\left(\alpha_{\delta}^{\prime}, \lambda\right) \\
& \mathbf{P}(\alpha) \quad \underset{\text { id }}{\hookrightarrow} \quad \mathbf{P}_{\gamma}(\lambda)
\end{aligned}
$$

commutes.

(Comment. This diagram implies that

$$
\begin{aligned}
& \mathbf{P}_{\gamma}(\lambda) *_{\text {id o icon }}{ }^{\left(\alpha^{\prime}, \alpha\right)} S^{\mathbf{P}\left(\alpha^{\prime}\right) * \mathbf{R}\left(\alpha^{\prime}, \alpha\right)}(\alpha, \lambda) \\
& =\mathbf{P}_{\gamma}(\lambda) *_{\text {icon }} \alpha_{\delta}^{\left(\alpha^{\prime}, \lambda\right) \circ \text { id }} \\
& \left.S^{\mathbf{P}\left(\alpha^{\prime}\right) * \mathbf{R}\left(\alpha^{\prime}, \alpha\right)}(\alpha, \lambda) .\right)
\end{aligned}
$$

For every $\mathbf{P}\left(\alpha^{\prime}\right) * \mathbf{R}\left(\alpha^{\prime}, \alpha\right) \in \Gamma_{\gamma}^{\prime}$, let $\tau_{\alpha^{\prime}}$ be the stage in the construction of $\mathbf{P}\left(\alpha^{\prime}\right) * \mathbf{R}\left(\alpha^{\prime}, \lambda\right)$ where $\alpha$ is handled. Note: $\tau_{\alpha^{\prime}} \geqslant \alpha$.

Case 1. For every $\mathbf{P}\left(\alpha^{\prime}\right) * \mathbf{R}\left(\alpha^{\prime}, \alpha\right) \in \Gamma_{\gamma}^{\prime}\left\langle\alpha^{\prime}, \tau_{\alpha^{\prime}}\right\rangle$ has been handled.

$\mathbf{P}_{\gamma}(\lambda)$ orders $\Gamma_{\gamma}^{\prime}$ in a natural way, namely the order in which the $\left\langle\alpha^{\prime}, \tau_{\alpha^{\prime}}\right\rangle$ have been handled. The order $<_{\alpha, \lambda}$ on $\Gamma_{\alpha}$ is defined by:

(i) every element of $\Gamma_{\gamma}^{\prime}$ is less than every element of $\Gamma_{\alpha} \sim \Gamma_{\gamma}^{\prime}$.

(ii) $\Gamma_{\gamma}^{\prime}$ is ordered by the order induced by $\mathbf{P}_{\gamma}(\lambda)$.

(iii) $\Gamma_{\alpha} \sim \Gamma_{\gamma}^{\prime}$ is canonically well ordered by its ordinal indices; i.e., if $\mathbf{Q}_{1}=$ $\mathbf{P}\left(\beta_{1}\right) * \mathbf{R}\left(\beta_{1}, \alpha\right) \in \Gamma_{\alpha}$ and $\mathbf{Q}_{2}=\mathbf{P}\left(\beta_{2}\right) * \mathbf{R}\left(\beta_{2}, \alpha\right) \in \Gamma_{\alpha}$ then $\mathbf{Q}_{1}<_{\alpha, \lambda} \mathbf{Q}_{2}$ iff $\beta_{1}<\beta_{2}$. Let

$$
\begin{gathered}
\xi_{0}=\text { o.t. }<_{\alpha, \lambda \uparrow} \Gamma_{\gamma}^{\prime}, \\
\xi_{1}=\text { o.t }<_{\alpha, \lambda} \uparrow \Gamma_{\alpha} \sim \Gamma_{\gamma}^{\prime} .
\end{gathered}
$$

Claim 1. P( $\alpha) * \mathbf{R}_{\xi_{0}}\left(\alpha, \lambda, \Gamma_{\alpha},<_{\alpha, \lambda}\right) \hookrightarrow \mathbf{P}_{\gamma}(\lambda)$.

Proof. We use Lemma 5.1 again. Enumerate $\Gamma_{\gamma}^{\prime}$ in the order $<_{\alpha, \beta}$, $\left\langle\mathbf{P}\left(\alpha_{\beta}\right) * \mathbf{R}\left(\alpha_{\beta}, \alpha\right): \beta<\xi_{0}\right\rangle$. Define $\psi$ as follows: Let $\psi(0)=\alpha$. For $\beta>0$ let $\psi(\beta)$ $=$ the stage where $\left\langle\alpha_{\beta}, \tau_{\alpha_{\beta}}\right\rangle$ has been handled. Since $\tau_{\alpha_{\beta}}>\alpha, \psi(\beta)>\alpha$ for all $\beta>0$. For $\eta \leqslant \xi_{0}$, we define

$$
\operatorname{icon}_{\eta}^{(\alpha, \lambda)}: \mathbf{P}(\alpha) * \mathbf{R}_{\eta}\left(\alpha, \lambda, \Gamma_{\alpha},<_{\alpha, \lambda}\right) \hookrightarrow \mathbf{P}_{\gamma}(\lambda)
$$

by $\operatorname{icon}_{\eta}^{(\alpha, \lambda)}(\sigma)=\sigma^{\prime}$ where

$$
\sigma^{\prime}(\delta)= \begin{cases}\sigma(0)(\delta) & \text { if } \delta<\alpha, \\ \sigma(\beta) & \text { if } \delta=\psi(\beta) \text { for } \delta \geqslant \alpha, \\ 1 & \text { if } \delta \geqslant \alpha \text { and } \delta \notin \psi^{\prime \prime} \eta .\end{cases}
$$

Subclaim. For all $\eta \leqslant \xi_{0}$,

$$
\operatorname{icon}_{\eta}^{(\alpha, \lambda)}: \mathbf{P}(\alpha) * \mathbf{R}_{\eta}\left(\alpha, \lambda, \Gamma_{\alpha},<_{\alpha, \lambda}\right) \hookrightarrow \mathbf{P}_{\gamma}(\lambda) \text {. }
$$

Proof. We go by induction. If $\eta=0$ then $\operatorname{icon}_{0}^{(\alpha, \lambda)}: \mathbf{P}(\alpha) \hookrightarrow \mathbf{P}_{\gamma}(\lambda)$ since icon ${ }_{0}^{(\alpha, \lambda)}$ $=\mathrm{id}$. Assume for all $\eta^{\prime}<\eta$ the subclaim is true. If $\eta$ is a successor ordinal then the lemma is trivial using Lemma 2.3. If $\eta$ is a limit then we want to use Lemma 5.1. As usual the only nontrivial condition to verify is the support criterion. 
Let $X \subseteq \eta, X$ a support for some condition in $\mathbf{P}(\alpha) * \mathbf{R}_{\eta}\left(\alpha, \lambda, \Gamma_{\alpha},<_{\alpha, \lambda}\right)$. We want that $\psi^{\prime \prime} X$ is a support in $\mathbf{P}_{\gamma}(\lambda)$. By our definition of type $\alpha$, every stage where an $\left\langle\alpha^{\prime}, \tau_{\dot{\alpha}^{\prime}}\right\rangle$ has been handled is of type $\alpha$.

Since $X$ is a support for $\mathbf{P}(\alpha) * \mathbf{R}_{\eta}\left(\alpha, \lambda, \Gamma_{\alpha},<_{\alpha, \lambda}\right),|X|<\alpha$. Thus, $\left|\psi^{\prime \prime} X\right|<\alpha$ and $\psi^{\prime \prime} X$ is bounded below $\gamma \cdot \psi^{\prime \prime} X \subseteq$ type $\alpha \cup\{\alpha\}$ so by P1 - P3, for all $\mu$ under attack,

$$
\left|\psi^{\prime \prime} X \cap(\operatorname{type}(\mathbf{P}(\mu), 0) \cup \operatorname{type}(\mathbf{P}(\mu), 1))\right| \leqslant 2 .
$$

Thus, $\psi^{\prime \prime} X$ is a support in $\mathbf{P}_{\delta}(\lambda)$ where $\delta=\sup \psi^{\prime \prime} X$.

Suppose $X \subseteq \eta$ and $\psi^{\prime \prime} X$ is a support for $\mathbf{P}_{\gamma}(\lambda)$. Then, since $\psi^{\prime \prime} X \subseteq$ type $\alpha \cup\{\boldsymbol{\alpha}\}$, $\left|\psi^{\prime \prime} X\right|<\alpha$. Thus $|X|<\alpha$ and $X$ is a support for $\mathbf{P}(\alpha) * \mathbf{R}_{\eta}\left(\alpha, \lambda, \Gamma_{\alpha},<_{\alpha, \lambda}\right)$. This establishes the subclaim which directly implies the claim.

Let $\mathbf{P}_{\gamma+\xi_{1}}(\lambda)$ be the result of iterating in the rest of $\mathbf{P}(\alpha) * \mathbf{R}_{\xi_{0}+\xi_{1}}(\alpha, \lambda)$ in the order $<_{\alpha, \lambda}$ with support of cardinality $<\boldsymbol{\alpha}$. Then we get a canonical

$$
\operatorname{icon}_{\xi_{0}+\xi_{1}}^{(\alpha, \lambda)}: \mathbf{P}(\alpha) * \mathbf{R}_{\xi_{0}+\xi_{1}}(\alpha, \lambda) \hookrightarrow \mathbf{P}_{\gamma+\xi_{1}}(\lambda)
$$

satisfying the induction hypothesis $\mathrm{H}$.

We call $\psi^{\prime \prime} \xi_{0} \cup\left[\gamma, \gamma+\xi_{1}\right)$ the type $(\mathbf{P}(\alpha), 0)$ coordinates and $\left[\gamma, \gamma+\xi_{1}\right)$ are all called type $\alpha$ coordinates. For each $\beta<\xi_{0}$, we say that $\langle\alpha, \beta\rangle$ is handled at stage $\psi(\beta)$. If $\beta=\xi_{0}+\eta<\xi_{0}+\xi_{1}$, then $\langle\alpha, \beta\rangle$ is said to be handled at stage $\gamma+\eta$. It is now easy to verify $\mathrm{P} 1-\mathrm{P} 5$. Finally, $\mathbf{P}(\alpha)$ is said to be under attack at this stage.

Case 2. Otherwise.

$$
\mathbf{P}_{\gamma+1}(\lambda)=\mathbf{R}_{\gamma}(\lambda) * 2
$$

and $\gamma$ is not type $\alpha^{\prime}$, type $\left(\mathbf{P}\left(\alpha^{\prime}\right), 0\right)$ or $\left(\mathbf{P}\left(\alpha^{\prime}\right), 1\right)$ for any $\alpha^{\prime}$.

We let $\mathbf{P}(\lambda)=\mathbf{P}(\lambda)=\lim \left(\mathbf{P}_{\alpha}(\lambda): \alpha<\lambda\right)$. This completes the definition of $\mathbf{P}(\lambda)$.

We now remark that if $\overrightarrow{\mathbf{P}(\alpha)} \rightarrow \mathbf{P}(\lambda)$, then

$$
\mathbf{P}(\alpha) * \mathbf{R}\left(\alpha, \lambda, \Gamma_{\alpha},<_{\alpha, \lambda}\right) \underset{\text { icon } n_{\lambda}^{(\alpha, \lambda)}}{\stackrel{P}{\text { id }}} \mathbf{P}(\lambda)
$$

for a canonically defined icon ${ }_{\lambda}^{(\alpha, \lambda)}$. (We will drop the subscript and write icon ${ }^{(\alpha, \lambda)}$.) This is because $\mathbf{P}(\alpha) \underset{\text { id }}{\hookrightarrow} \mathbf{P}(\lambda)$ implies that $\mathbf{P}(\alpha) \underset{\text { id }}{\hookrightarrow} \mathbf{P}_{\alpha}(\lambda)$. Thus $\mathbf{P}(\alpha)$ comes up $\lambda$ times in Case c. At stages $\gamma$ where $\mathbf{P}(\alpha)$ is considered, look at $\Gamma_{\gamma}^{\prime}$. If $\gamma_{1}<\gamma_{2}$ then $\Gamma_{\gamma_{1}}^{\prime} \subseteq \Gamma_{\gamma_{2}}^{\prime}$. Since the cardinality of $\Gamma_{\alpha}$ is small, eventually $\Gamma_{\gamma}^{\prime}$ stabilizes to some $\Gamma^{\prime}$. Since $\Gamma^{\prime}$ has small cardinality there is a $\delta$ such that all the relevant $\left\langle\alpha^{\prime}, \tau_{\alpha^{\prime}}\right\rangle$ have been handled by stage $\delta$. The next time $\mathbf{P}(\alpha)$ comes up in Case c, it comes under attack. Once $\mathbf{P}(\alpha)$ is under attack, we use the induction hypothesis together with Lemma 5.1 to see that there is a canonical

$$
\operatorname{icon}^{(\alpha, \lambda)}: \mathbf{P}(\alpha) * \mathbf{R}\left(\alpha, \lambda, \Gamma_{\alpha},<_{\alpha, \lambda}\right) \hookrightarrow \mathbf{P}(\lambda) .
$$

We now state a lemma whose proof is an easy but tedious application of the recursion theorem.

Lemma 6.1. (a) For any $Q \subseteq V_{\alpha}(\alpha$ Mahlo) that is $\alpha$ c.c. and any $X,<$,

$$
\mathbf{Q} * \mathbf{R}(\alpha, \lambda, X,<) \subseteq V_{\lambda}
$$

and is first order definable in $V_{\lambda+\omega}$ with parameters $Q, \alpha, \lambda, X,<$. 
(b) For any Mahlo $\alpha, \mathbf{P}(\alpha) \subseteq V_{\alpha}$ and is first order definable in $V_{\alpha+\omega}$ with $\alpha$ as a parameter.

(c) For any Mahlo $\alpha$ and $\lambda,<_{\alpha, \lambda}, \Gamma_{\alpha}$ and $\mathbf{P}(\alpha) * \mathbf{R}\left(\alpha, \lambda, \Gamma_{\alpha},<_{\alpha, \lambda}\right)$ are first order definable with parameters $\alpha$ and $\lambda$.

(d) For any $\alpha, \alpha^{\prime}, \beta$ the map $\operatorname{icon}_{\beta}^{\left(\alpha, \alpha^{\prime}\right)}$ is definable with $\alpha, \alpha^{\prime}$ and $\beta$ as parameters.

LEMMA 6.2. ${ }^{2}$

(a) If $\lambda$ is Mahlo then $\mathbf{P}(\lambda)$ has the $\lambda$ c.c.

(b) If $\kappa, \lambda$ are Mahlo and $Q \subseteq V_{\kappa}$ has the $\kappa$ c.c. then $\mathbf{Q} * \mathbf{R}(\kappa, \lambda, X,<)$ has the $\lambda$ c.c.

Proof. (a). Claim. If $\mathbf{P}_{\alpha}(\lambda)$ has the $\lambda$ c.c. for all $\alpha<\lambda$, then $\mathbf{P}(\lambda)$ has the $\lambda$ c.c.

Proof. Let $\left\langle p_{\lambda}: \gamma\langle\lambda\rangle\right.$ be an arbitrary $\lambda$ sequence of conditions from $\mathbf{P}(\lambda)$. We need to show that two conditions in this sequence are compatible.

For each $\gamma$, let $f(\gamma)=\sup \left(\operatorname{supp} p_{\gamma} \cap \gamma\right)$. $f$ is regressive on inaccessibles (since we took direct limits at inaccessibles) and $\gamma$ is Mahlo so there is a stationary set $S \subseteq \lambda$ such that $f$ is constant on $S$, say with constant value $\beta$. We can thin $S$ to a set $S^{\prime}$ of cardinality $\lambda$ with the property that if $\alpha_{1}, \alpha_{2} \in S^{\prime}$

$$
\operatorname{supp} p_{\alpha_{1}} \cap \operatorname{supp} p_{\alpha_{2}} \subseteq \beta .
$$

By the $\lambda$ c.c. of $\mathbf{P}_{\beta}(\lambda)$ there are $\gamma_{1}, \gamma_{2} \in S^{\prime}$ such that $p_{\gamma_{1}} \uparrow \beta$ and $p_{\gamma_{2}} \uparrow \beta$ are compatible. The supports of $p_{\gamma_{1}}$ and $p_{\gamma_{2}}$ are disjoint beyond $\beta$ so we can define a condition $q$ by picking $q$ i $\beta$ such that

$$
q \text { i } \beta \leqslant p_{\gamma_{1}} \uparrow \beta, p_{\gamma_{2}} \uparrow \beta
$$

and for $\alpha \geqslant \beta$, defining $q$ by

$$
q(\alpha)= \begin{cases}p_{\gamma_{1}}(\alpha) & \text { if } p_{\gamma_{2}}(\alpha)=1 \\ p_{\gamma_{2}}(\alpha) & \text { if } p_{\gamma_{1}}(\alpha)=1\end{cases}
$$

$q$ is well defined and $\operatorname{supp} q=\operatorname{supp} p_{\gamma_{1}} \cup \operatorname{supp} p_{\gamma_{2}} \cup \operatorname{supp} q \uparrow \beta$, hence $q$ really is a condition. It is easy to see that $q \leqslant p_{\gamma_{1}}, p_{\gamma_{2}}$ because $q \uparrow \beta \leqslant p_{\gamma_{1}} \uparrow \beta \wedge p_{\gamma_{2}} \uparrow \beta$ and for any $\alpha>\beta, 1 H q(\alpha)<p_{\gamma_{1}}(\alpha) \wedge p_{\gamma_{2}}(\alpha)$ since at most one of $p_{\gamma_{1}}(\alpha)$ and $p_{\gamma_{2}}(\alpha)$ is not 1 and $q(\alpha)$ takes on that value.

From the claim it suffices to see that $\mathbf{P}_{\alpha}(\lambda)$ has the $\lambda$ c.c. for all $\alpha<\lambda$. Let $\left\langle p_{\gamma}: \gamma\langle\lambda\rangle\right.$ be an arbitrary subset of $\mathbf{P}_{\alpha}(\lambda)$. We want to show that there are $\gamma_{1}, \gamma_{2}$ such that $p_{\gamma_{1}}$ and $p_{\gamma_{2}}$ are compatible.

Since $\lambda$ is inaccessible, $|\mathcal{P}(\alpha)|<\lambda$. Thus by a pigeonhole argument we can assume that for all $\gamma_{1}, \gamma_{2}, \operatorname{supp} p_{\gamma_{1}}=\operatorname{supp} p_{\gamma_{2}}$. Let $X \subseteq \alpha$ be this common support.

For each $\delta \in X, \mathbf{P}_{\delta+1}(\lambda)=\mathbf{P}_{\delta}(\lambda) *_{i_{\delta}} S^{\mathbf{Q}_{\delta}}\left(\lambda_{\delta}, \lambda\right)$, for some $i_{\delta}$ and $\mathbf{Q}_{\delta} \subseteq V_{\lambda_{\delta}}$. Since $\mathbf{Q}_{\delta}$ has the $\lambda_{\delta}^{+}$c.c., for each $\gamma$ we can find a set $D_{\gamma, \delta}$ in $V$ of cardinality $\lambda_{\delta}$ such that

\footnotetext{
$\aleph_{n}$.

${ }^{2}$ The argument here is essentially $R$. Laver's chain condition argument for an $\boldsymbol{\aleph}_{n+1}$ saturated ideal on
} 
$\mathbf{Q}_{\delta} \| \operatorname{dom} p_{\gamma}(\boldsymbol{\delta}) \subseteq D_{\gamma, \delta} \times \lambda_{\delta}$. (Recall, $\mathbf{Q}_{\delta} \| p_{\gamma}(\boldsymbol{\delta}) \in S^{\mathbf{Q}_{\delta}}\left(\lambda_{\delta}, \lambda\right)$, so $\mathbf{Q}_{\delta} \| \exists D,|D|=$ $\lambda_{\delta}$ such that $\operatorname{dom} p_{\gamma}(\delta) \subseteq D \times \lambda_{\delta}$.) Let $\eta=\sup _{\delta \in X} \lambda_{\delta}$. Define a function $f$ with domain $\lambda \sim \eta$ by

$$
f(\gamma)=\sup \left(\bigcup_{\delta \in X} D_{\gamma, \delta} \cap \gamma\right) .
$$

Again we see that $f$ is regressive on inaccessible $\gamma$. Since $\lambda$ is Mahlo, there is a stationary set $S$ and an ordinal $\beta<\lambda$ such that for all $\gamma \in S, f(\gamma)=\beta$. Pick $S^{\prime} \subseteq S$ of cardinality $\lambda$ such that for all $\gamma_{1}, \gamma_{2} \in S^{\prime}$

$$
\left(\bigcup_{\delta \in X} D_{\gamma_{1}, \delta}\right) \cap\left(\bigcup_{\delta \in X} D_{\gamma_{2}, \delta}\right) \subseteq \beta
$$

(Without loss of generality we assume $\beta>\eta$.)

For each $\gamma \in S^{\prime}$ we build a new condition $q_{\gamma}$ canonically by making

$$
\left\|q_{\gamma}(\delta)=p_{\gamma}(\delta) \uparrow\left(D_{\gamma, \delta} \cap \beta\right) \times \lambda_{\delta}\right\|=1 .
$$

We notice that $\mathbf{Q}_{\delta} \# q_{\gamma}(\delta) \in S\left(\lambda_{\delta}, \beta\right)$. Since $\left|\mathbf{Q}_{\delta}\right|=\lambda_{\delta}$ there can be at most $2^{\beta}$ distinct $q_{\gamma}(\boldsymbol{\delta})$ up to equivalence in $V^{\mathbf{Q}_{\delta}}$. Computing we get $\mid\left\{\left\langle q_{\gamma}(\delta): \delta \in X\right\rangle \mid \gamma \in\right.$ $\left.S^{\prime}\right\} \mid \leqslant\left(2^{\beta}\right)^{|X|}<\lambda$. Thus there must be $\gamma_{1}$ and $\gamma_{2} \in S^{\prime}$ such that $\left\langle q_{\gamma_{1}}(\delta): \delta \in X\right\rangle=$ $\left\langle q_{\gamma_{2}}(\delta): \delta \in X\right\rangle$. (Again up to equivalence.)

CLAIM. $p_{\gamma_{1}}$ and $p_{\gamma_{2}}$ are compatible.

Proof. It is enough to see that for all $\delta \in X, \mathbf{Q}_{\delta} \mathbb{H} p_{\gamma_{1}}(\delta)$ and $p_{\gamma_{2}}(\delta)$ are compatible. But $\mathbf{Q}_{\delta} I t \operatorname{dom} p_{\gamma_{1}}(\delta) \cap \operatorname{dom} p_{\gamma_{2}}(\delta) \subseteq \operatorname{dom} q_{\gamma_{1}}(\delta)$ and $q_{\gamma_{1}}(\delta)=q_{\gamma_{2}}(\delta)$. Thus $\mathbf{Q}_{\delta} H$ $p_{\gamma_{1}}(\delta) \cup p_{\gamma_{2}}(\delta)$ is a condition, because $p_{\gamma_{1}}(\delta)$ and $p_{\gamma_{2}}(\delta)$ are equal everywhere in the intersection of their domains.

This proves part (a).

The proof of part (b) is very similar.

7. The master condition. Our final partial order is

$$
\mathbf{P}\left(\kappa_{0}\right) * \mathbf{R}\left(\kappa_{0}, \kappa_{1}, \Gamma_{\kappa_{0}},<_{\kappa_{0}, \kappa_{1}}\right) * \bar{S}\left(\kappa_{0}, \kappa_{1}\right) .
$$

Lemma 7.1. For every $\mathbf{Q}=\mathbf{P}(\alpha) * \mathbf{R}\left(\alpha, \kappa_{0}, \Gamma_{\alpha},<_{\alpha, \kappa_{0}}\right)$ with $\left(\mathbf{Q}\right.$, icon $\left.^{\left(\alpha, \kappa_{0}\right)}\right) \in \Gamma_{\kappa_{0}}$, there are $k_{1}$ and $k_{2}$ such that the following diagram commutes:

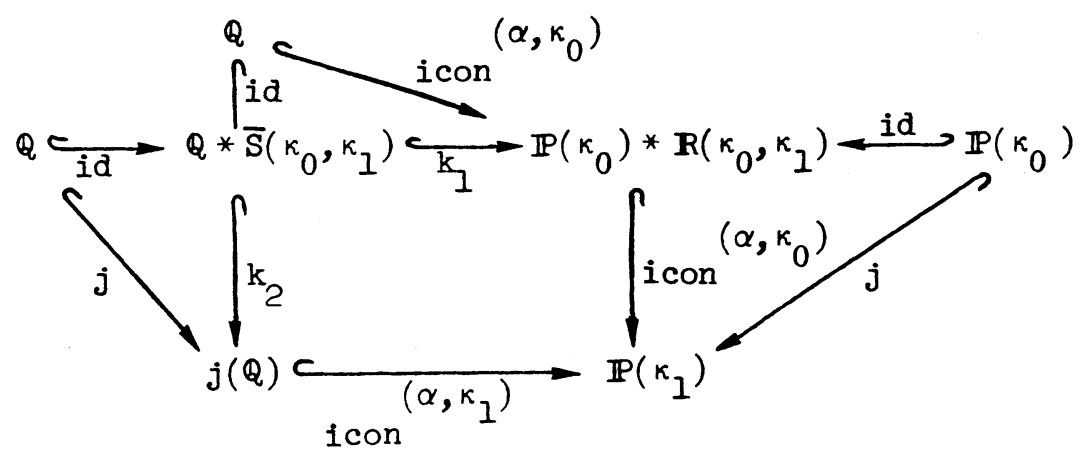

(see diagram in Lemma 3.1). 
Proof. Fix such an $\alpha$. Since $\left(\mathbf{P}(\alpha) * \mathbf{R}\left(\alpha, \kappa_{0}\right)\right.$, icon $\left.{ }^{\left(\alpha, \kappa_{0}\right)}\right) \in \Gamma_{\kappa_{0}}$, there is a stage $\beta$ where $\mathbf{P}(\alpha) * \mathbf{R}\left(\alpha, \kappa_{0}\right)$ is handled in the first phase of the construction of $\mathbf{P}\left(\kappa_{0}\right) * \mathbf{R}\left(\kappa_{0}, \kappa_{1}\right)$. Let $k_{1}$ be the map that sends $(q, s) \in \mathbf{Q} * \bar{S}\left(\kappa_{0}, \kappa_{1}\right)$ to $\sigma \in$ $\mathbf{P}\left(\kappa_{0}\right) * \mathbf{R}\left(\kappa_{0}, \kappa_{1}\right)$, where $\sigma$ is defined by:

$$
\begin{aligned}
& \sigma(0)=\operatorname{icon}^{\left(\alpha, \kappa_{0}\right)}(q), \\
& \sigma(\delta)= \begin{cases}1 & \text { unless } \delta=\beta, \\
s & \text { if } \delta=\beta .\end{cases}
\end{aligned}
$$

As we remarked after the definition of $\mathbf{Q} * \mathbf{R}(\kappa, \lambda, X,<)$ the map defined this way is a neat embedding.

Since $\mathbf{Q}=\mathbf{P}(\alpha) * \mathbf{R}\left(\alpha, \kappa_{0}, \Gamma_{\alpha},<_{\alpha, \kappa_{0}}\right)$ is first order definable with parameters $\alpha$ and $\kappa_{0}, j(Q)=\mathbf{P}(\alpha) * \mathbf{R}\left(\alpha, \kappa_{1}, \Gamma_{1},<_{\alpha, \kappa_{1}}\right)$. By Lemma 6.2, $\mathbf{Q}$ has the $\kappa_{0}$ c.c. Thus

$$
j: \mathbf{P}(\alpha) * \mathbf{R}\left(\alpha, \kappa_{0}, \Gamma_{\alpha},<_{\alpha, \kappa_{0}}\right) \hookrightarrow \mathbf{P}(\alpha) * \mathbf{R}\left(\alpha, \kappa_{1}, \Gamma_{\alpha},<_{\alpha, \kappa_{1}}\right)
$$

is a neat embedding. Since $\mathbf{P}(\alpha) * \mathbf{R}\left(\alpha, \kappa_{0}\right) \subseteq V_{\kappa_{0}}, j \uparrow \mathbf{P}(\alpha) * \mathbf{R}\left(\alpha, \kappa_{0}, \Gamma_{\alpha},<_{\alpha, \kappa_{o}}\right)$ is the identity on $\mathbf{P}(\alpha) * \mathbf{R}\left(\alpha, \kappa_{0}\right)$. We note that $<_{\alpha, \kappa_{0}}=<_{\alpha, \kappa_{1}}$, since $\left\{\Gamma_{\alpha_{1}},<_{\alpha, \kappa_{0}}\right\} \in V_{\kappa_{0}}$ and so $<_{\alpha, \kappa_{0}}=j\left(<_{\alpha, \kappa_{0}}\right)=<_{\alpha, \kappa_{1}}$. Summing up we get that

$$
\text { id: } \mathbf{P}(\alpha) * \mathbf{R}\left(\alpha, \kappa_{0}, \Gamma_{\alpha},<_{\alpha, \kappa_{0}}\right) \hookrightarrow \mathbf{P}(\alpha) * \mathbf{R}\left(\alpha, \kappa_{1}, \Gamma_{\alpha},<_{\alpha, \kappa_{1}}\right) \text {. }
$$

From this we deduce that there is a stage $\tau \geqslant \kappa_{0}$ in the second phase of the construction where $\kappa_{0}$ is handled. We let

$$
k_{2}: \mathbf{P}(\alpha) * \mathbf{R}\left(\alpha, \kappa_{0}\right) * \bar{S}\left(\kappa_{0}, \kappa_{1}\right) \rightarrow \mathbf{P}(\alpha) * \mathbf{R}\left(\alpha, \kappa_{1}\right)
$$

be the map defined as follows: for $p \in \mathbf{P}(\alpha) * \mathbf{R}\left(\alpha, \kappa_{0}\right)$ and $s \in \bar{S}\left(\kappa_{0}, \kappa_{1}\right)$,

$$
k_{2}((p, s))=\sigma \text { where } \sigma(\eta)= \begin{cases}p(\eta) & \text { if } \eta<\kappa_{0} \\ s & \text { if } \eta=\tau \\ 1 & \text { otherwise }\end{cases}
$$

Again by our remarks in $\S 6, k_{2}$ is a neat embedding, and it is now easy to check that the following diagram commutes:

$$
\mathbf{P}(\alpha) * \mathbf{R}\left(\alpha, \kappa_{0}\right) \stackrel{\text { id }}{\Leftrightarrow} \mathbf{P}(\alpha) * \mathbf{R}\left(\alpha, \kappa_{0}\right) * \bar{S}\left(\kappa_{0}, \kappa_{1}\right)
$$

We now turn our attention to showing that the central square of our diagram commutes. Since $\mathbf{P}\left(\kappa_{0}\right)$ has the $\kappa_{0}$ c.c. and $\mathbf{P}\left(\kappa_{0}\right) \subseteq V_{\kappa_{0}}$, id: $\mathbf{P}\left(\kappa_{0}\right) \hookrightarrow \mathbf{P}\left(\kappa_{1}\right)$ and hence $\mathbf{P}\left(\kappa_{0}\right)$ comes under attack at some stage $\gamma \geqslant \kappa_{0}$ in the construction of $\mathbf{P}\left(\kappa_{1}\right)$.

Claim. Let $\gamma$ be the stage in the construction of $\mathbf{P}\left(\kappa_{1}\right)$ where $\mathbf{P}\left(\kappa_{0}\right)$ comes under attack, then $\mathbf{P}(\alpha) * \mathbf{R}\left(\alpha, \kappa_{0}, \Gamma_{\alpha},<_{\alpha, \kappa_{0}}\right) \in \Gamma_{\gamma}^{\prime}$.

Proof. We verify (1)-(5) in the definition of $\Gamma_{\gamma}^{\prime}$

(1) is trivial since $\alpha<\kappa_{0}<\kappa$.

(2) Since $\mathbf{P}(\alpha) \hookrightarrow \mathbf{P}\left(\kappa_{0}\right), \mathbf{P}(\alpha)$ comes under attack in the construction of $\mathbf{P}\left(\kappa_{0}\right)$ at some stage $\eta<\kappa_{0}$. By elementarity, $j(\eta)$ is the stage where $\mathbf{P}(j(\alpha))$ comes under 
attack in the construction of $\mathbf{P}\left(\kappa_{1}\right)$. But $j(\eta)=\eta$ and $j(\alpha)=\alpha$, hence $\eta$ is the stage where $\mathbf{P}(\alpha)$ comes under attack in the construction of $\mathbf{P}\left(\kappa_{1}\right)$. Since $\gamma \geqslant \kappa_{0}>\eta, \mathbf{P}(\alpha)$ is under attack at stage $\gamma$.

(3) We have argued previously that $<_{\alpha, \kappa_{0}}=<_{\alpha, \kappa_{1}}$.

(4) We have to check that

$$
\text { id: } \mathbf{P}(\alpha) * \mathbf{R}\left(\alpha, \kappa_{0}\right) \hookrightarrow \mathbf{P}(\alpha) * \mathbf{R}_{\delta}\left(\alpha, \kappa_{1}\right)
$$

where $\delta$ is the greatest ordinal for which

$$
\operatorname{icon}_{\delta}^{\left(\alpha, \kappa_{1}\right)}: \mathbf{P}(\alpha) * \mathbf{R}_{\delta}\left(\alpha, \kappa_{1}\right) \rightarrow \mathbf{P}_{\gamma}\left(\kappa_{1}\right)
$$

is defined.

Since $\gamma \geqslant \kappa_{0}$, and for all $\beta<\kappa_{0}$, icon $\cos _{\beta}^{\left(\alpha, \kappa_{0}\right)}$ is defined, again by elementarity we see that for all $\beta<\kappa_{0}$, icon $\cos ^{\left(\alpha, \kappa_{1}\right)}$ is defined. Thus $\delta \geqslant \kappa_{0}$. We know that

$$
\text { id: } \mathbf{P}(\alpha) * \mathbf{R}\left(\alpha, \kappa_{0}\right) \hookrightarrow \mathbf{P}(\alpha) * \mathbf{R}\left(\alpha, \kappa_{1}\right) .
$$

$\mathbf{P}(\alpha) * \mathbf{R}\left(\alpha, \kappa_{0}\right)$ is an iteration of length $\kappa_{0}$, so id: $\mathbf{P}(\alpha) * \mathbf{R}\left(\alpha, \kappa_{0}\right) \hookrightarrow$ $\mathbf{P}(\alpha) * \mathbf{R}_{\kappa_{0}}\left(\alpha, \kappa_{1}\right)$. Thus

$$
\text { id: } \mathbf{P}(\alpha) * \mathbf{R}\left(\alpha, \kappa_{0}\right) \hookrightarrow \mathbf{P}(\alpha) * \mathbf{R}_{\delta}\left(\alpha, \kappa_{1}\right) .
$$

This establishes (4).

(5) We must check that

$$
\begin{array}{ccc}
\mathbf{P}(\alpha) * \mathbf{R}\left(\alpha, \kappa_{0}\right) & \hookrightarrow & \mathbf{P}(\alpha) * \mathbf{R}_{\delta}\left(\alpha, \kappa_{1}\right) \\
\int_{\text {icon }}\left(\alpha, \kappa_{0}\right) & & \int_{\text {icon }}^{\left(\alpha, \kappa_{1}\right)} \\
\mathbf{P}\left(\kappa_{0}\right) & \hookrightarrow & \mathbf{P}_{\gamma}\left(\kappa_{1}\right)
\end{array}
$$

commutes.

Let $p \in \mathbf{P}(\alpha) * \mathbf{R}\left(\alpha, \kappa_{0}\right)$. Then $j(p)=\mathrm{id}(p)$ so $j(p) \in \mathbf{P}(\alpha) * \mathbf{R}_{\kappa_{0}}\left(\alpha, \kappa_{1}\right)$. By Lemma 6.1

$$
j\left(\operatorname{icon}^{\left(\alpha, \kappa_{0}\right)}(p)\right)=\operatorname{icon}^{\left(\alpha, \kappa_{1}\right)}(j(p))=\left(\operatorname{icon}^{\left(\alpha, \kappa_{1}\right)} \circ \mathrm{id}\right)(p) .
$$

Since $\mathbf{P}\left(\kappa_{0}\right) \subset V_{\kappa_{0}}, j \uparrow \mathbf{P}\left(\kappa_{0}\right)=$ id.

From this we conclude

$$
j\left(\operatorname{icon}^{\left(\alpha, \kappa_{0}\right)}(p)\right)=\left(\operatorname{id} \circ \operatorname{icon}^{\left(\alpha, \kappa_{0}\right)}\right)(p) .
$$

Summing up: $\left(\mathrm{id} \circ \operatorname{icon}^{\left(\alpha, \kappa_{0}\right)}\right)(p)=\left(\operatorname{icon}^{\left(\alpha, \kappa_{1}\right)} \circ \mathrm{id}\right)(p)$. This completes the proof of the claim.

Let $\psi$ be the map defined at the stage where $\mathbf{P}\left(\kappa_{0}\right)$ comes under attack. Let $\tau$ be the stage in the construction of $\mathbf{P}(\alpha) * \mathbf{R}\left(\alpha, \kappa_{1}\right)$ where $\kappa_{0}$ is handled. Let $\beta$ be the stage in the first phase of $\mathbf{P}\left(\kappa_{0}\right) * \mathbf{R}\left(\kappa_{0}, \kappa_{1}\right)$ where $\left\langle\mathbf{P}(\alpha) * \mathbf{R}\left(\alpha, \kappa_{0}\right)\right.$, icon $\left.{ }^{\left(\alpha, \kappa_{0}\right)}\right\rangle$ is handled. Then $\psi(\beta)=$ the stage where $\langle\alpha, \tau\rangle$ is handled. (Since $\tau>\kappa_{0}, \psi(\beta)>\kappa_{0}$.)

Let us consider a typical element $\langle r, \sigma\rangle \in \mathbf{P}(\alpha) * \mathbf{R}\left(\alpha, \kappa_{0}\right) * \bar{S}\left(\kappa_{0}, \kappa_{1}\right)$, where $r \in \mathbf{P}(\alpha) * \mathbf{R}\left(\alpha, \kappa_{0}\right)$ and $\sigma \in \bar{S}\left(\kappa_{0}, \kappa_{1}\right)$. 
We want to follow it around the central square of the diagram:

$$
\begin{array}{ccc}
\mathbf{P}(\alpha) * \mathbf{R}\left(\alpha, \kappa_{0}\right) * \bar{S}\left(\kappa_{0}, \kappa_{1}\right) & \underset{k_{1}}{\hookrightarrow} & \mathbf{P}\left(\kappa_{0}\right) * \mathbf{R}\left(\kappa_{0}, \kappa_{1}\right) \\
\int_{k_{2}} & & \int_{\text {icon }^{\left(\kappa_{0}, \kappa_{1}\right)}} \\
\mathbf{P}(\alpha) * \mathbf{R}\left(\alpha, \kappa_{1}\right) & \underset{j\left(\text { icon }^{\left(\alpha, \kappa_{0}\right)}\right)}{\hookrightarrow} & \mathbf{P}\left(\kappa_{1}\right)
\end{array}
$$

As we remarked before

$$
j\left(\operatorname{icon}^{\left(\alpha, \kappa_{0}\right)}\right)=\operatorname{icon}^{\left(\alpha, \kappa_{1}\right)}
$$

$k_{1}(r, \sigma)=\sigma^{\prime}$ where

$$
\sigma^{\prime}(\eta)= \begin{cases}\operatorname{icon}^{\left(\alpha, \kappa_{0}\right)}(r) & \text { if } \eta=0 \\ \sigma & \text { if } \eta=\beta \\ 1 & \text { otherwise }\end{cases}
$$

and $\operatorname{icon}^{\left(\kappa_{0}, \kappa_{1}\right)}\left(\sigma^{\prime}\right)=\sigma^{*}$ where $\sigma^{*} \uparrow \kappa_{0}=\operatorname{icon}^{\left(\alpha, \kappa_{0}\right)}(r)$ and for $\eta \geqslant \kappa_{0}$,

$$
\sigma^{*}(\eta)= \begin{cases}\sigma & \text { if } \eta=\psi(\beta) \\ 1 & \text { otherwise. }\end{cases}
$$

(This is because icon ${ }^{\left(\kappa_{0}, \kappa_{1}\right)}, \mathbf{P}\left(\kappa_{0}\right)=$ id.)

Going the other way around the diagram,

$k_{2}(r, \sigma)=\varphi$ where:

$$
\varphi(\eta)= \begin{cases}r(\eta) & \text { if } \eta<\kappa_{0} \\ \sigma & \text { if } \eta=\tau \\ 1 & \text { otherwise }\end{cases}
$$

Note that $\varphi \uparrow \kappa_{0}=r$.

We have to see that $\operatorname{icon}^{\left(\alpha, \kappa_{1}\right)}(\varphi)=\sigma^{*}$. Now $\operatorname{icon}^{\left(\alpha, \kappa_{1}\right)}(\varphi)=\operatorname{icon}^{\left(\alpha, \kappa_{1}\right)}\left(\varphi \mid \kappa_{0}\right) \cup \varphi^{*}$ where

$$
\varphi^{*}(\eta)= \begin{cases}\sigma & \text { if } \eta=\text { the stage where }\langle\alpha, \tau\rangle \text { is handled, } \\ 1 & \text { otherwise. }\end{cases}
$$

$\left(\varphi^{*}\right.$ defined only for $\eta \geqslant \kappa_{0}$.)

Because $r \in V_{\kappa_{0}}$ and $\operatorname{icon}^{\left(\alpha, \kappa_{0}\right)}(r) \in V_{\kappa_{0}}$ and Lemma 6.1,

$$
\begin{aligned}
\operatorname{icon}^{\left(\alpha, \kappa_{0}\right)}(r) & =j\left(\operatorname{icon}^{\left(\alpha, \kappa_{0}\right)}(r)\right) \\
& =\operatorname{icon}^{\left(\alpha, \kappa_{1}\right)}(j(r)) \\
& =\operatorname{icon}^{\left(\alpha, \kappa_{1}\right)}(r) .
\end{aligned}
$$

Thus we can conclude that

$$
\operatorname{icon}^{\left(\alpha, \kappa_{1}\right)}\left(\varphi \uparrow \kappa_{0}\right)=\operatorname{icon}^{\left(\alpha, \kappa_{1}\right)}(r)=\operatorname{icon}^{\left(\alpha, \kappa_{0}\right)}(r)=\sigma^{*} \uparrow \kappa_{0} .
$$

There is only one element in each of the supports of $\operatorname{icon}^{\left(\alpha, \kappa_{1}\right)}(\varphi)$ and $\sigma^{*}$ beyond $\kappa_{0}$. These are the stage where $\langle\alpha, \tau\rangle$ is handled and $\psi(\beta)$ respectively. But by the definition of $\psi, \psi(\beta)=$ the stage where $\langle\alpha, \tau\rangle$ is handled. At this coordinate, $\sigma^{*}$ and 
$\operatorname{icon}^{\left(\alpha, \kappa_{1}\right)}(\varphi)$ both have value $\sigma$. Thus, $\sigma^{*}$ and $\operatorname{icon}^{\left(\alpha, \kappa_{1}\right)}(\varphi)$ agree beyond $\kappa_{0}$ and hence $\sigma^{*}=\operatorname{icon}^{\left(\alpha, \kappa_{1}\right)}(\varphi)$. This completes the proof that the central square commutes.

The other triangles of the diagram are easily seen to commute. This establishes the lemma.

Since $j: \mathbf{P}\left(\kappa_{0}\right) \hookrightarrow \mathbf{P}\left(\kappa_{1}\right)$ is the identity,

$$
\mathbf{P}\left(\kappa_{0}\right) * \mathbf{R}\left(\kappa_{0}, \kappa_{1}\right) \in \Gamma_{\kappa_{1}}
$$

and we get a map $i$ such that the following diagram commutes:

$$
\begin{gathered}
\mathbf{P}\left(\kappa_{0}\right) * \mathbf{R}\left(\kappa_{0}, \kappa_{1}\right) \underset{\text { id }}{\rightarrow} \mathbf{P}\left(\kappa_{0}\right) * \mathbf{R}\left(\kappa_{0}, \kappa_{1}\right) * \bar{S}\left(\kappa_{1}, \kappa_{2}\right) \\
\operatorname{icon}^{\left(\kappa_{0}, \kappa_{1}\right)} \int_{i} \\
\mathbf{P}\left(\kappa_{1}\right) * \mathbf{R}\left(\kappa_{1}, \kappa_{2}\right) .
\end{gathered}
$$

We can view $i$ as

$$
i: \mathbf{P}\left(\kappa_{0}\right) * \mathbf{R}\left(\kappa_{0}, \kappa_{1}\right) * \bar{S}\left(\kappa_{1}, \kappa_{2}\right) \hookrightarrow \mathbf{P}\left(\kappa_{1}\right) * \mathbf{R}\left(\kappa_{1}, \kappa_{2}\right) * \bar{S}\left(\kappa_{2}, \kappa_{3}\right) .
$$

We want to build a master condition for $i$ and $j$ in the sense of $\S 3$.

Our master condition will be built by first creating a master condition for $\operatorname{icon}^{\left(\kappa_{0}, \kappa_{1}\right):} \mathbf{P}\left(\kappa_{0}\right) * \mathbf{R}\left(\kappa_{0}, \kappa_{1}\right) \hookrightarrow \mathbf{P}\left(\kappa_{1}\right)$ and $j: \mathbf{P}\left(\kappa_{0}\right) * \mathbf{R}\left(\kappa_{0}, \kappa_{1}\right) \rightarrow \mathbf{P}\left(\kappa_{1}\right) * \mathbf{R}\left(\kappa_{1}, \kappa_{2}\right)$. After we have constructed this we will adjoin the master condition for the Silver collapse.

The idea here is to build the master condition, $p_{1}$, coordinate by coordinate, using Lemma 7.1 so that at each coordinate $\alpha, p_{1}(\alpha)$ acts as a master condition for that coordinate.

For each $\alpha$,

$$
\mathbf{P}\left(\kappa_{1}\right) * \mathbf{R}_{\alpha+1}\left(\kappa_{1}, \kappa_{2}\right)=\mathbf{P}\left(\kappa_{1}\right) * \mathbf{R}_{\alpha}\left(\kappa_{1}, \kappa_{2}\right) *_{e_{\alpha}} S^{\mathbf{Q}_{\alpha}}\left(\lambda_{\alpha}, \kappa_{2}\right)
$$

for some $\mathbf{Q}_{\alpha}, e_{\alpha}$ and $\lambda_{\alpha}$. Let $\pi_{\alpha}: \mathscr{B}\left(\mathbf{P}\left(\kappa_{1}\right) * \mathbf{R}\left(\kappa_{1}, \kappa_{2}\right)\right) \rightarrow \mathscr{B}\left(\mathbf{Q}_{\alpha}\right)$ be the projection map splitting $e_{\alpha}$. (Similarly for each $\beta<\kappa_{1}, \mathbf{P}\left(\kappa_{0}\right) * \mathbf{R}_{\beta+1}\left(\kappa_{0}, \kappa_{1}\right)=$ $\mathbf{P}\left(\kappa_{0}\right) * \mathbf{R}_{\beta}\left(\kappa_{0}, \kappa_{1}\right) *_{e_{\beta}} S^{\mathbf{Q}_{\beta}}\left(\lambda_{\beta}, \kappa_{1}\right)$ for some $e_{\beta}, \mathbf{Q}_{\beta}$ and $\lambda_{\beta}$. We will call the projection map $\pi_{\mathbf{Q}_{\beta}}: \mathfrak{B}\left(\mathbf{P}\left(\kappa_{0}\right) * \mathbf{R}_{\beta}\left(\kappa_{0}, \kappa_{1}\right)\right) \rightarrow \mathscr{B}\left(\mathbf{Q}_{\beta}\right)$.) Let $\gamma=$ o.t. $<_{\kappa_{0}, \kappa_{1}}$ on $\Gamma_{\kappa_{0}}$. Then $j(\gamma)=$ o.t. $<_{\kappa_{1}, \kappa_{2}}$. If $\alpha<j(\gamma)$ then either $\mathbf{Q}_{\alpha}=\mathbf{P}(\beta) * \mathbf{R}\left(\beta, \kappa_{1}\right)\left(\beta<\kappa_{1}\right)$ and $\lambda_{\alpha}=\kappa_{1}$ or $\mathbf{Q}_{\alpha}=\mathbf{P}\left(\kappa_{1}\right)$. If $\alpha \geqslant j(\gamma)$, then $\mathbf{Q}_{\alpha}=\mathbf{P}\left(\kappa_{1}\right) * \mathbf{R}\left(\kappa_{1}, \lambda_{\alpha}\right)$ for some $\lambda_{\alpha}>\kappa_{1}$.

Let $A=j^{\prime \prime} \gamma$. If $p \in \mathbf{P}\left(\kappa_{0}\right) * \mathbf{R}\left(\kappa_{0}, \kappa_{1}\right)$ then $|\operatorname{supp} p \cap \gamma|<\kappa_{0}$, so $j(\operatorname{supp} p \cap \gamma)$ $=j^{\prime \prime}(\operatorname{supp} p \cap \gamma)$. Thus supp $j(p) \cap j(\gamma)=j^{\prime \prime}(\operatorname{supp} p \cap \gamma)$. This is important, because it means that in order to build a master condition for the first phase of $\mathbf{P}\left(\kappa_{0}\right) * \mathbf{R}\left(\kappa_{0}, \kappa_{1}\right)$ we need only consider coordinates in $A$. If $\alpha \in A$ and $\mathbf{Q}_{\alpha}=$ $\mathbf{P}(\beta) * \mathbf{R}\left(\beta, \kappa_{1}\right)$ for some $\beta<\kappa_{1}$ then $\alpha=j\left(\alpha^{\prime}\right)$ for some $\alpha^{\prime}<\gamma$ and $\mathbf{Q}_{\alpha}=j\left(\mathbf{Q}^{\prime}\right)$ for some $\mathbf{Q}^{\prime}=\mathbf{P}\left(\beta^{\prime}\right) * \mathbf{R}\left(\beta^{\prime}, \kappa_{0}\right)$. The only way for this to happen is if $\beta=\beta^{\prime}$. Hence for $\alpha \in A$ we know that $\mathbf{Q}_{\alpha}=\mathbf{P}(\beta) * \mathbf{R}\left(\beta, \kappa_{1}\right)$ for some $\beta<\kappa_{0}$, or $\mathbf{Q}_{\alpha}=\mathbf{P}\left(\kappa_{1}\right)$. If 
$\mathbf{Q}_{\alpha}=\mathbf{P}(\beta) * \mathbf{R}\left(\beta, \kappa_{1}\right)$ for some $\beta<\kappa_{0}$ then by Lemma 7.1 there are $k_{1}^{\alpha}$ and $k_{2}^{\alpha}$ such that the following commutes:

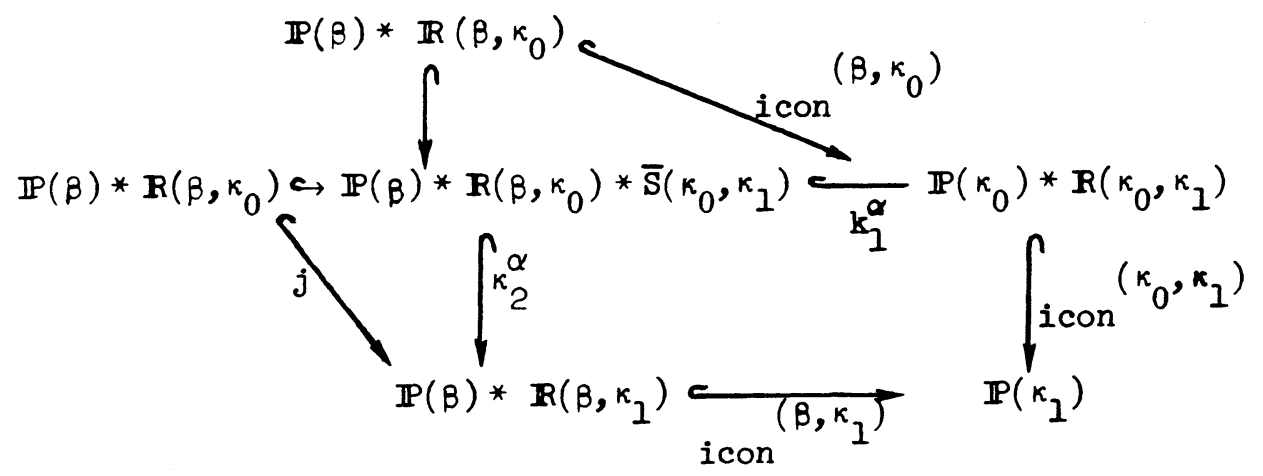

We argue analogously to Lemma 3.1. Let $\dot{H}$ be a term in $V^{\mathbf{P}(\beta) * \mathbf{R}\left(\beta, \kappa_{1}\right)}$ denoting the generic object for $\mathbf{P}(\beta) * \mathbf{R}\left(\beta, \kappa_{0}\right) * \bar{S}\left(\kappa_{0}, \kappa_{1}\right)$ defined via $k_{2}^{\alpha}$ in $V^{\mathbf{P}(\beta) * \mathbf{R}\left(\beta, \kappa_{1}\right)}$. There is a term $\tau$ in

$$
S^{\mathbf{P}(\beta) * \mathbf{R}\left(\beta, \kappa_{1}\right)}\left(\kappa_{1}, \kappa_{2}\right)
$$

defined in $V^{\mathbf{P}(\beta) * \mathbf{R}\left(\beta, \kappa_{1}\right)}$ by

$$
\tau=\bigcup_{\substack{\langle q, s\rangle \in H \\ q \in \mathbf{P}(\beta) * \mathbf{R}\left(\boldsymbol{\beta}, \kappa_{0}\right) \\ s \in \bar{S}\left(\kappa_{0}, \kappa_{1}\right)}} j(s) .
$$

Then, as in Lemma 3.1, for all $q \in \mathbf{P}(\beta) * \mathbf{R}\left(\beta, \kappa_{0}\right) * \bar{S}\left(\kappa_{0}, \kappa_{1}\right),\langle 1, \tau\rangle \wedge k_{2}^{\alpha}(q) \leqslant$ $j(q) .\left(\langle 1, \tau\rangle\right.$ is considered to be an element of $\mathbf{P}(\beta) * \mathbf{R}\left(\beta, \kappa_{1}\right) * \bar{S}\left(\kappa_{1}, \kappa_{2}\right)$.) Call this term $\tau_{\alpha}$. If $\mathbf{Q}_{\alpha}=\mathbf{P}\left(\kappa_{1}\right)$ then there are $k_{1}^{\alpha}$ and $k_{2}^{\alpha}$ such that the following commutes:

$$
\begin{array}{rcc}
\mathbf{P}\left(\kappa_{0}\right) * \bar{S}\left(\kappa_{0}, \kappa_{1}\right) \underset{k_{1}^{\alpha}}{\stackrel{\mathbf{p}}{k_{2}^{\alpha}}} & \mathbf{P}\left(\kappa_{0}\right) * \mathbf{R}\left(\kappa_{0}, \kappa_{1}\right) \\
& \int_{\text {icon }}\left(\kappa_{0}, \kappa_{1}\right) \\
& \mathbf{P}\left(\kappa_{1}\right) .
\end{array}
$$

So by Kunen's theorem there is a $\tau \in S^{\mathbf{P}\left(\kappa_{1}\right)}\left(\kappa_{1}, \kappa_{2}\right)$ such that for all $q \in$ $\mathbf{P}\left(\kappa_{0}\right) * \bar{S}\left(\kappa_{0}, \kappa_{1}\right),(1, \tau) \wedge k_{2}^{\alpha}(q) \leqslant j(q)$. In this case, call this term $\tau_{\alpha}$.

We define our master condition $p_{1}$ for $j: \mathbf{P}\left(\kappa_{0}\right) * \mathbf{R}\left(\kappa_{0}, \kappa_{1}\right) \rightarrow \mathbf{P}\left(\kappa_{1}\right) * \mathbf{R}\left(\kappa_{1}, \kappa_{2}\right)$ inductively: For $\alpha<j(\gamma)$, let

$$
p_{1}(\alpha)= \begin{cases}1 & \text { if } \alpha=0, \\ \tau_{\alpha} & \text { if } \alpha \in A, \\ 1 & \text { if } \alpha \notin A .\end{cases}
$$

The supp $p_{1}\left\lceil j(\gamma)\right.$ has cardinality the cardinality of $j^{\prime \prime} \gamma$ which is less than $\kappa_{1}$, so $p_{1} \mid j(\gamma) \in \mathbf{P}\left(\kappa_{1}\right) * \mathbf{R}\left(\kappa_{1}, \kappa_{2}\right)$.

We need to introduce some notation: If $\sigma \in V^{\mathbf{Q}_{\alpha}}$ and $\mathbf{Q}_{\alpha} H \sigma \in S^{\mathbf{Q}_{\alpha}}\left(\lambda_{\alpha}, \kappa_{1}\right)$ we let $\underline{\sigma}$ be the condition in $\mathbf{P}\left(\kappa_{1}\right) * \mathbf{R}\left(\kappa_{1}, \kappa_{2}\right)$ defined to be $\sigma$ at $\alpha$ and 1 everywhere else.

We now show that if $H^{\prime} \subseteq \mathbf{P}\left(\kappa_{1}\right) * \mathbf{R}\left(\kappa_{1}, \kappa_{2}\right)$ is generic and $p_{1} \uparrow j(\gamma) \in H^{\prime}$ then for all $\sigma \in \mathbf{P}\left(\kappa_{0}\right) * \mathbf{R}\left(\kappa_{0}, \kappa_{1}\right)$ such that icon ${ }^{\left(\kappa_{0}, \kappa_{1}\right)}(\sigma) \in H^{\prime}, j(\sigma) \uparrow j(\gamma) \in H^{\prime}$. We will do 
this by showing that if $\operatorname{icon}^{\left(\kappa_{0}, \kappa_{1}\right)}(\sigma) \in H^{\prime}$ and $\alpha<j(\gamma)$ then $j(\sigma)(\alpha) \in H^{\prime}$. This suffices since $H^{\prime}$ is a complete ultrafilter on $\mathscr{B}\left(\mathbf{P}\left(\kappa_{1}\right) * \mathbf{R}\left(\kappa_{1}, \kappa_{2}\right)\right)$.

Suppose $\operatorname{icon}^{\left(\kappa_{0}, \kappa_{1}\right)}(\sigma) \in H^{\prime}$. Then as we argued earlier, $\operatorname{supp} j(\sigma) \cap j(\gamma)=$ $j^{\prime \prime}(\operatorname{supp} \sigma \cap \gamma)$. Let $\alpha \in \operatorname{supp} j(\sigma) \cap j(\gamma)$. Then $\alpha=j\left(\alpha^{\prime}\right)$ for some $\alpha^{\prime}<\gamma$ and $j(\sigma)(\alpha)=j\left(\sigma\left(\alpha^{\prime}\right)\right)$. If $\alpha=0$, icon ${ }^{\left(\kappa_{0}, \kappa_{1}\right)}(\sigma(0))=\sigma(0)=j(\sigma(0))$, since $\sigma(0) \in \mathbf{P}\left(\kappa_{0}\right)$. If $\alpha \neq 0$ and $\mathbf{Q}_{\alpha}=\mathbf{P}(\beta) * \mathbf{R}\left(\beta, \kappa_{1}\right)$ for some $\beta<\kappa_{0}$ we consider:

$$
\begin{array}{ccc}
\mathbf{P}(\beta) * \mathbf{R}\left(\beta, \kappa_{0}\right) * \bar{S}\left(\kappa_{0}, \kappa_{1}\right) & \underset{k_{1}^{\alpha}}{\hookrightarrow} & \mathbf{P}\left(\kappa_{0}\right) * \mathbf{R}\left(\kappa_{0}, \kappa_{1}\right) \\
\underset{\int_{2}^{\alpha}}{ } & \underset{\operatorname{icon}^{\left(\beta, \kappa_{1}\right)}}{\hookrightarrow} & \mathbf{P}\left(\kappa_{1}\right) .
\end{array}
$$

By the proof of Lemma 3.1, $k_{2}^{\alpha}\left(1, \sigma\left(\alpha^{\prime}\right)\right) \wedge\left(1, \tau_{\alpha}\right) \leqslant(1, j(\sigma)(\alpha))$ in $\mathbf{P}(\beta) * \mathbf{R}\left(\beta, \kappa_{1}\right) * \bar{S}\left(\kappa_{1}, \kappa_{2}\right)$. (Here we are considering $1 \in \mathbf{P}(\beta) * \mathbf{R}\left(\beta, \kappa_{1}\right)$ and $\tau_{\alpha} \in$ $\bar{S}\left(\kappa_{1}, \kappa_{2}\right)$.) In other words $k_{2}^{\alpha}\left(1, \sigma\left(\alpha^{\prime}\right)\right) \| \tau_{\alpha} \leqslant j(\sigma)(\alpha)$.

ClaIM. icon ${ }^{\left(\kappa_{0}, \kappa_{1}\right)}(\sigma) \wedge \tau_{\alpha} \leqslant j(\sigma)(\alpha)$.

Proof. This is true iff $\frac{\alpha}{\pi_{\alpha}}\left(\mathrm{icon}^{\left(\kappa_{0}, \kappa_{1}\right)}(\sigma)\right) \mathbb{H}_{\mathbf{Q}_{\alpha}} \tau_{\alpha} \leqslant j(\sigma)(\alpha)$. Since the diagram commutes,

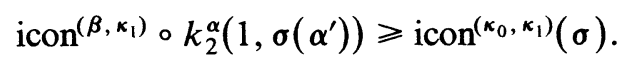

But this implies that $\pi_{\alpha}\left(\operatorname{icon}^{\left(\kappa_{0}, \kappa_{1}\right)}(\sigma)\right) \leqslant k_{2}^{\alpha}\left(1, \sigma\left(\alpha^{\prime}\right)\right)$. Hence $\pi_{\alpha}\left(\operatorname{icon}^{\left(\kappa_{0}, \kappa_{1}\right)}(\sigma)\right) \sharp_{\mathbf{Q}_{\alpha}} \tau_{\alpha}$ $\leqslant j(\sigma)(\alpha)$ as desired.

The only case remaining is where $\mathbf{Q}_{\alpha}=\mathbf{P}\left(\kappa_{1}\right)$. Since

$$
\begin{aligned}
& \mathbf{P}\left(\kappa_{0}\right) * \bar{S}\left(\kappa_{0}, \kappa_{1}\right) \underset{k_{1}^{\alpha}}{\hookrightarrow} \quad \mathbf{P}\left(\kappa_{0}\right) * \mathbf{R}\left(\kappa_{0}, \kappa_{1}\right) \\
& \mathbf{P}\left(\kappa_{1}\right)
\end{aligned}
$$

commutes,

$$
\operatorname{icon}^{\left(\kappa_{0}, \kappa_{1}\right)}(\sigma) H_{\mathbf{P}\left(\kappa_{1}\right)} \tau_{\alpha} \leqslant j(\sigma)(\alpha) \text { in } S^{\mathbf{P}\left(\kappa_{1}\right)}\left(\kappa_{1}, \kappa_{2}\right)
$$

by Kunen's theorem, and hence icon ${ }^{\left(\kappa_{0}, \kappa_{1}\right)}(\sigma) \wedge \tau_{\alpha} \leqslant \underline{j(\sigma)(\alpha)}$. This shows what we wanted since $\underline{\tau_{\alpha}} \geqslant p_{1} \mid \gamma$.

To define $\overline{p_{1}}$ beyond $j(\gamma)$ :

Let

$$
B=\bigcup_{q \in \mathbf{P}\left(\kappa_{0}\right) * \mathbf{R}\left(\kappa_{0}, \kappa_{1}\right)} j(\operatorname{supp}(q)) .
$$

Then $|B|=\left|\kappa_{1} \times \kappa_{1}\right|=\kappa_{1}$ and $B \cap j(\gamma)=A$. The support of $p_{1}$ will be $B$. Each $\alpha \in B \sim \gamma$ is the stage where $\mathbf{Q}_{\alpha}=\mathbf{P}\left(\kappa_{1}\right) * \mathbf{R}\left(\kappa_{1}, \lambda_{\alpha}\right)$ is handled for some $\lambda_{\alpha}>\kappa_{1}$. To define $p(\alpha)$, we assume by induction that $p_{1} \uparrow \alpha$ has been defined and if $q \in \mathbf{P}\left(\kappa_{0}\right) * \mathbf{R}\left(\kappa_{0}, \kappa_{1}\right)$ then $\operatorname{icon}^{\left(\kappa_{0}, \kappa_{1}\right)}(q) \wedge p_{1} \mid \alpha \leqslant j(q) \uparrow \alpha$. (Note that we already have this for $p_{1} \uparrow \gamma$.)

Let $G \in V^{\mathbf{P}\left(\kappa_{1}\right) * \mathbf{R}\left(\kappa_{1}, \kappa_{2}\right)}$ be a term for the generic object for $\mathbf{P}\left(\kappa_{0}\right) * \mathbf{R}\left(\kappa_{0}, \kappa_{1}\right)$ defined by icon ${ }^{\left(\kappa_{0}, \kappa_{1}\right)}$. 
There is a term $G_{\alpha}$ in $V^{\mathbf{P}\left(\kappa_{1}\right) * \mathbf{R}\left(\kappa_{1}, \lambda_{\alpha}\right)}$ with the property that

$$
\left\|G_{\boldsymbol{\alpha}}^{\mathbf{P}\left(\kappa_{1}\right) * \mathbf{R}\left(\kappa_{1}, \lambda_{\alpha}\right)}=G\right\|_{\mathbf{P}\left(\kappa_{1}\right) * \mathbf{R}\left(\kappa_{1}, \kappa_{2}\right)}=1 .
$$

(Here the boolean value is taken in $\mathscr{B}\left(\mathbf{P}\left(\kappa_{1}\right) * \mathbf{R}\left(\kappa_{1}, \kappa_{2}\right)\right.$ ) and

$$
G_{\alpha}^{\mathbf{P}\left(\kappa_{1}\right) * \mathbf{R}\left(\kappa_{1}, \lambda_{\alpha}\right)}
$$

means the denotation of $G_{\alpha}$ in

$$
V^{\mathbf{P}\left(\kappa_{1}\right) * \mathbf{R}\left(\kappa_{1}, \lambda_{\alpha}\right)} \text {. }
$$

Recall $\mathbf{P}\left(\kappa_{1}\right) * \mathbf{R}\left(\kappa_{1}, \lambda_{\alpha}\right) \underset{\text { id }}{\hookrightarrow} \mathbf{P}\left(\kappa_{1}\right) * \mathbf{R}\left(\kappa_{1}, \kappa_{2}\right)$ so this makes sense.) We will define $p_{1}(\alpha)$ to be a term

$$
\tau \in S^{\mathbf{P}\left(\kappa_{1}\right) * \mathbf{R}\left(\kappa_{1}, \lambda_{\alpha}\right)}\left(\lambda_{\alpha}, \kappa_{2}\right)
$$

with the property that $\pi_{\alpha}\left(p_{1} \mid \alpha\right) \forall_{\mathbf{Q}_{\alpha}}$ for every $q \in G_{\alpha}, \quad \tau<j(q)(\alpha)$ in $S^{\mathbf{P}\left(\kappa_{1}\right) * \mathbf{R}\left(\kappa_{1}, \lambda_{\alpha}\right)}\left(\lambda_{\alpha}, \kappa_{2}\right)$. This will suffice to show the induction hypothesis at $\alpha$, since to check that

$$
\operatorname{icon}^{\left(\kappa_{0}, \kappa_{1}\right)}(q) \wedge p_{1} \uparrow(\alpha+1) \leqslant j(q) \uparrow(\alpha+1)
$$

we need that

(1) $\operatorname{icon}^{\left(\kappa_{0}, \kappa_{1}\right)}(q) \wedge p_{1} \uparrow \alpha \leqslant j(q) \uparrow \alpha$ and

(2) $\pi_{\alpha}\left(\operatorname{icon}^{\left(\kappa_{0}, \kappa_{1}\right)}(q) \wedge p_{1} \uparrow \alpha\right) H_{\mathbf{Q}_{\alpha}} j(q)(\alpha) \geqslant p_{1}(\alpha)$.

But since

$$
G_{\alpha}^{\mathbf{P}\left(\kappa_{1}\right) * \mathbf{R}\left(\kappa_{1}, \lambda_{\alpha}\right)}=G^{\mathbf{P}\left(\kappa_{1}\right) * \mathbf{R}\left(\kappa_{1}, \kappa_{2}\right)},
$$

then

$$
\pi_{\alpha}\left(\operatorname{icon}^{\left(\kappa_{0}, \kappa_{1}\right)}(q) \wedge p_{1} \uparrow \alpha\right) \mathbb{q} \in G_{\alpha} .
$$

Since $\pi_{\alpha}\left(p_{1} \uparrow \alpha\right) \Vdash \forall q \in G_{\alpha},\left(p_{1}(\alpha)<j(q)(\alpha)\right)$, we get

$$
\pi_{\alpha}\left(\operatorname{icon}^{\left(\kappa_{0}, \kappa_{1}\right)}(q) \wedge p_{1} \uparrow \alpha\right) H p_{1}(\alpha)<j(q)(\alpha) .
$$

We must show that there is such a $\tau$. In $V^{\mathbf{P}\left(\kappa_{1}\right) * \mathbf{R}\left(\kappa_{1}, \lambda_{\alpha}\right)}$, we let $\tau=\cup_{q \in G_{\alpha}} j(q)(\alpha)$.

LEMMA 7.2. $\pi_{\alpha}\left(p_{1} \mid \alpha\right) \Vdash \tau \in S^{\mathbf{P}\left(\kappa_{1}\right) * \mathbf{R}\left(\kappa_{1}, \lambda_{\alpha}\right)}\left(\lambda_{\alpha}, \kappa_{2}\right)$.

Proof. We start with a claim.

Claim. $\pi_{\alpha}\left(p_{1} \mid \boldsymbol{\alpha}\right)$ H for all $q_{1}, q_{2} \in G_{\alpha}, j\left(q_{1}\right)(\alpha)$ and $j\left(q_{2}\right)(\alpha)$ are compatible.

Proof. Let $H \subseteq \mathbf{P}\left(\kappa_{1}\right) * \mathbf{R}\left(\kappa_{1}, \kappa_{2}\right)$ be generic with $p_{1} \uparrow \alpha \in H$. Then for all $q \in$ $\mathbf{P}\left(\kappa_{0}\right) * \mathbf{R}\left(\kappa_{0}, \kappa_{1}\right), q \in G_{\alpha}$ iff $\operatorname{icon}^{\left(\kappa_{0}, \kappa_{1}\right)}(q) \in H$. Let $q_{1}$ and $q_{2} \in G$. There is a $q_{3} \in G$ such that $q_{3} \leqslant q_{1}, q_{2}$. This means that for all $\beta, \pi_{\mathbf{Q}_{\beta}}\left(q_{3} \uparrow \beta\right) \mathbb{H} q_{1}(\beta)$ and $q_{2}(\beta)$ are compatible. (Here $\mathbf{Q}_{\beta}$ is the partial ordering corresponding to the $\beta$ th coordinate of $\mathbf{P}\left(\kappa_{0}\right) * \mathbf{R}\left(\kappa_{0}, \kappa_{1}\right)$ and $\pi_{\mathbf{Q}_{\beta}}$ is the projection onto $\mathbf{Q}_{\beta}$.) By elementarity $\pi_{\alpha}\left(j\left(q_{3}\right) \uparrow \alpha\right) \Vdash q_{1}(\alpha)$ and $q_{2}(\alpha)$ are compatible. But by the induction hypothesis, $j\left(q_{3}\right) \uparrow \alpha \in H$, since icon $^{\left(\kappa_{0}, \kappa_{1}\right)}\left(q_{3}\right) \wedge p_{1} \uparrow \alpha \leqslant j\left(q_{3}\right) \uparrow \alpha$. Hence, if $H^{\prime}$ is the generic object for $\mathbf{Q}_{\alpha}$ induced by $e_{\alpha}$, then $V\left[H^{\prime}\right] \vDash q_{1}(\alpha)$ and $q_{2}(\alpha)$ are compatible. This proves the claim.

To see the lemma, we note that $|G|=\kappa_{1}$. Since $\mathbf{P}\left(\kappa_{1}\right) * \mathbf{R}\left(\kappa_{1}, \lambda_{\alpha}\right)$ has the $\lambda_{\alpha}$ c.c., $\lambda_{\alpha}$ is a cardinal in $V^{\mathbf{P}\left(\kappa_{1}\right) * \mathbf{R}\left(\kappa_{1}, \lambda_{\alpha}\right)}$. Thus $\pi\left(p_{1} \uparrow \alpha\right) \Vdash \tau$ is the union of $<\lambda_{\alpha}$ compatible elements of $S\left(\lambda_{\alpha}, \kappa_{1}\right)$ and thus $\pi\left(p_{1} \uparrow \alpha\right) \Vdash \tau \in S\left(\lambda_{\alpha}, \kappa_{1}\right)$. 
This proves the lemma.

It is now easy to see that $\tau$ is the term we desired. We need that $\pi_{\alpha}(p \nmid \alpha)$ Ht for all $q \in G_{\alpha}, \tau<j(q)(\alpha)$. But

$$
\pi_{\alpha}(p \nmid \alpha) H \tau=\bigcup_{q \in G_{\alpha}} j(q)(\alpha)
$$

hence for any $q \in G_{\alpha}, \pi_{\alpha}(p \nmid \alpha) \Vdash \tau<j(q)(\alpha)$.

The induction hypothesis carries over automatically at limit stages.

Let $p_{1}$ be the result of carrying out this process through all of $B$. We get from our induction that:

If $q \in \mathbf{P}\left(\kappa_{0}\right) * \mathbf{R}\left(\kappa_{0}, \kappa_{1}\right)$ then icon ${ }^{\left(\kappa_{0}, \kappa_{1}\right)}(q) \wedge p_{1} \leqslant j(q)$. This was our definition of a master condition for icon ${ }^{\left(\kappa_{0}, \kappa_{1}\right)}$ and $j$.

Let $\hat{G}$ be a generic object for $\mathbf{P}\left(\kappa_{1}\right) * \mathbf{R}\left(\kappa_{1}, \kappa_{2}\right)$ with $p_{1} \in \hat{G}$. Let $G$ be the induced generic object for $\mathbf{P}\left(\kappa_{0}\right) * \mathbf{R}\left(\kappa_{0}, \kappa_{1}\right)$ via icon ${ }^{\left(\kappa_{0}, \kappa_{1}\right)}$. Since $p_{1}$ is a master condition we get that for all $q \in G, j(q) \in \hat{G}$. Since $G$ is an ultrafilter and $j$ preserves incompatibility, for all $q \in \mathbf{P}\left(\kappa_{0}\right) * \mathbf{R}\left(\kappa_{0}, \kappa_{1}\right), q \in G$ iff $j(q) \in \hat{G}$. Thus we get an elementary embedding $\hat{j}$ extending $j$ such that

$$
\dot{\hat{j}}: V[G] \rightarrow M[\hat{G}] .
$$

Because $\mathbf{P}\left(\kappa_{0}\right) * \mathbf{R}\left(\kappa_{0}, \kappa_{1}\right) \in \Gamma_{\kappa_{1}}$ there is a map $i$,

$$
i: \mathbf{P}\left(\kappa_{0}\right) * \mathbf{R}\left(\kappa_{0}, \kappa_{1}\right) * \bar{S}\left(\kappa_{1}, \kappa_{2}\right) \rightarrow \mathbf{P}\left(\kappa_{1}\right) * \mathbf{R}\left(\kappa_{1}, \kappa_{2}\right)
$$

extending icon ${ }^{\left(\kappa_{0}, \kappa_{1}\right)}$. In $V^{\mathbf{P}\left(\kappa_{1}\right) * \mathbf{R}\left(\kappa_{1}, \kappa_{2}\right)}$ we have a term $\dot{G}$ for a generic object on $\mathbf{P}\left(\kappa_{0}\right) * \mathbf{R}\left(\kappa_{0}, \kappa_{1}\right) * \bar{S}\left(\kappa_{1}, \kappa_{2}\right)$ induced by $i$. Let $\tau \in V^{\mathbf{P}\left(\kappa_{1}\right) * \mathbf{R}\left(\kappa_{1}, \kappa_{2}\right)}$ be defined by

$$
\tau=\bigcup_{\substack{\left\langle q, \boldsymbol{\sigma} \backslash \in G \\ q \in \mathbf{P}\left(\kappa_{0}\right) * \mathbf{R}\left(\kappa_{0}, \kappa_{1}\right) \\ \boldsymbol{\sigma} \in \bar{S}\left(\kappa_{1}, \kappa_{2}\right)\right.}} j(\boldsymbol{\sigma}) .
$$

The usual arguments show that

$$
p_{1} H \tau \in S^{\mathbf{P}\left(\kappa_{1}\right) * \mathbf{R}\left(\kappa_{1}, \kappa_{2}\right)}\left(\kappa_{2}, \kappa_{3}\right)
$$

and that $p_{1} \mathbb{H} \tau<j(\sigma)$ for all $\langle q, \sigma\rangle \in G$. Thus if $\langle q, \sigma\rangle \in$ $\left(\mathbf{P}\left(\kappa_{0}\right) * \mathbf{R}\left(\kappa_{0}, \kappa_{1}\right)\right) * \bar{S}\left(\kappa_{1}, \kappa_{2}\right)$ then $i(q, \sigma) \wedge\left\langle p_{1}, \tau\right\rangle \leqslant(j(q), j(\sigma))$. This establishes that $\left\langle p_{1}, \tau\right\rangle$ is a master condition for $i$ and $j$. Let $p=\left\langle p_{1}, \tau\right\rangle$.

Let us sum up what we have done. We started with $j: V \rightarrow M$ a 2-huge embedding. We found partial orders $\mathbf{P}=\mathbf{P}\left(\kappa_{0}\right) * \mathbf{R}\left(\kappa_{0}, \kappa_{1}\right) * \bar{S}\left(\kappa_{1}, \kappa_{2}\right)$, and $j(\mathbf{P})=$ $\mathbf{P}\left(\kappa_{1}\right) * \mathbf{R}\left(\kappa_{1}, \kappa_{2}\right) * \bar{S}\left(\kappa_{2}, \kappa_{3}\right)$ such that there is a map $i: \mathbf{P} \hookrightarrow j(\mathbf{P})$ and a master condition $p$ for $i$ and $j$. By the definition of a master condition, we know that if $\hat{G}$ is $j(\mathbf{P})$-generic with $p \in \hat{G}$ and $G$ is the generic object induced on $\mathbf{P}$, then for all $q \in \mathbf{P}$, $q \in G$ iff $j(q) \in \hat{G}$. From this we know that we can extend $j$ to an elementary embedding $\dot{\hat{j}}: V[G] \rightarrow M[\hat{G}]$.

LEMMA 7.3. In $V^{\mathbf{P}}, \kappa_{0}=\aleph_{1}, \kappa_{1}=\aleph_{2}$ and $\kappa_{2}=\aleph_{3}$.

Proof. Since $\mathbf{P}_{0}\left(\kappa_{0}\right)=S\left(\omega, \kappa_{0}\right), \kappa_{0} \leqslant \boldsymbol{\kappa}_{1}$ in $V^{\mathbf{P}}$. We only need to observe that $\kappa_{0}$ is the first ordinal moved by $\hat{j}$ in order to see that $\kappa_{0}$ is a cardinal. 
We know that $\mathbf{P}\left(\kappa_{0}\right) \in \Gamma_{\kappa_{0}}$, hence $\mathbf{P}\left(\kappa_{0}\right) * \bar{S}\left(\kappa_{0}, \kappa_{1}\right) \hookrightarrow \mathbf{P}\left(\kappa_{0}\right) * \mathbf{R}\left(\kappa_{0}, \kappa_{1}\right)$. Thus $\kappa_{1} \leqslant \aleph_{2}^{V^{\mathbf{P}}}$. By the elementarity of $\dot{\hat{j}}, \kappa_{1}$ is a cardinal in $j(\mathbf{P})$. But $\mathbf{P} \rightarrow j(\mathbf{P})$ and so $\kappa_{1}$ is a cardinal in $V^{\mathbf{P}}$. Thus $\kappa_{1}=\boldsymbol{\kappa}_{2}^{V^{\mathbf{P}}}$.

We now have an elementary embedding $\dot{\hat{j}}: V[G] \rightarrow M[\hat{G}]$ such that $V[G] \vDash \kappa_{0}=\boldsymbol{\aleph}_{1}$, $\kappa_{1}=\aleph_{2}, \kappa_{2}=\aleph_{3}$. Reasoning as in $\S 1$, we see that $V[G] \vDash\left(\aleph_{3}, \kappa_{2}, \aleph_{1}\right) \rightarrow$ $\left(\aleph_{2}, \aleph_{1}, \aleph_{0}\right)$ and we have established:

\section{THEOREM.}

$$
\begin{aligned}
& \text { Con(ZFC }+ \text { there is a 2-huge cardinal }) \\
& \qquad \Leftrightarrow \operatorname{Con}\left(\mathrm{ZFC}+\left(\boldsymbol{\aleph}_{3}, \boldsymbol{\aleph}_{2}, \boldsymbol{\aleph}_{1}\right) \rightarrow\left(\boldsymbol{\aleph}_{2}, \boldsymbol{\aleph}_{1}, \boldsymbol{\aleph}_{0}\right)\right) .
\end{aligned}
$$

8. Final remarks. We now want to establish the consistency of $\left(\aleph_{3}, \aleph_{2}, \aleph_{1}\right) \rightarrow\left(\aleph_{2}, \aleph_{1}, \boldsymbol{\aleph}_{0}\right)$ with the G.C.H. and indicate the modifications necessary to get $\left(\boldsymbol{\aleph}_{n+3}, \boldsymbol{\aleph}_{n+2}, \boldsymbol{\aleph}_{n+1}\right) \rightarrow\left(\boldsymbol{\aleph}_{n+2}, \boldsymbol{\aleph}_{n+1}, \boldsymbol{\aleph}_{n}\right)$.

Lemma 8.1. Let $j: V \rightarrow M$ be a 2-huge embedding. Let $G$ be P-generic. Let $B \subseteq \kappa_{2}$ code all the bounded subsets of $\kappa_{2}$ in $V[G]$. Then $L[B]=\left(\boldsymbol{\aleph}_{3}, \boldsymbol{\aleph}_{2}, \boldsymbol{\aleph}_{1}\right) \rightarrow\left(\boldsymbol{\aleph}_{2}, \boldsymbol{\aleph}_{1}, \boldsymbol{\aleph}_{0}\right)$ + G.C.H.

Proof. By standard arguments the G.C.H. holds for all $\alpha \geqslant \kappa_{2}$ in $L[B]$. To see the G.C.H. in $L[B]$, it is enough to see that $V[G] \vDash 2^{\aleph_{0}}=\kappa_{0}, 2^{\kappa_{0}}=\kappa_{1}$ and $2^{\kappa_{1}}=\kappa_{2}$.

Let $\Phi_{\mathbf{P}}=\{A: A$ is an antichain in $\mathbf{P}\}$. It is a standard fact that

$$
V[G] \vDash|\mathcal{P}(\alpha)| \leqslant\left|\alpha \mathscr{G}_{\mathbf{P}} \cap V\right| \text {. }
$$

For $\alpha=\kappa_{1}: \mathbf{P}$ is $\kappa_{2}$ c.c. in $V$, so $\left|g=\kappa_{2}\right|$ in $V$. Since $\kappa_{2}$ is inaccessible, $\left|\kappa_{1} g_{\mathbf{P}}\right|=\kappa_{2}$. Thus $V[G] \vDash\left|\mathcal{P}\left(\kappa_{1}\right)\right| \leqslant \kappa_{2}$.

For $\alpha=\kappa_{0}: \operatorname{In} V^{\mathbf{P}\left(\kappa_{0}\right) * \mathbf{R}\left(\kappa_{0}, \kappa_{1}\right)}$,

$$
S^{\mathbf{P}\left(\kappa_{0}\right) * \mathbf{R}\left(\kappa_{0}, \kappa_{1}\right)}\left(\kappa_{1}, \kappa_{2}\right)
$$

is $<\kappa_{1}$ closed. Hence,

$$
\mathscr{P}\left(\kappa_{0}\right)^{V[G]}=\mathscr{P}\left(\kappa_{0}\right)^{V\left[G \mid \mathbf{P}\left(\kappa_{0}\right) * \mathbf{R}\left(\kappa_{0}, \kappa_{1}\right)\right]}
$$

and thus we only need to check $2^{\kappa_{0}}=\kappa_{1}$ in $V\left[G \uparrow \mathbf{P}\left(\kappa_{0}\right) * \mathbf{R}\left(\kappa_{0}, \kappa_{1}\right)\right]$. Again let $\oint_{\mathbf{P}\left(\kappa_{0}\right) * \mathbf{R}\left(\kappa_{0}, \kappa_{1}\right)}=\left\{A: A\right.$ is an antichain in $\left.\mathbf{P}\left(\kappa_{0}\right) * \mathbf{R}\left(\kappa_{0}, \kappa_{1}\right)\right\}$. Since $\mathbf{P}\left(\kappa_{0}\right) * \mathbf{R}\left(\kappa_{0}, \kappa_{1}\right)$ is $\kappa_{1}$ c.c.,

$$
\left|\oint_{\mathbf{P}\left(\kappa_{0}\right) * \mathbf{R}\left(\kappa_{0}, \kappa_{1}\right)}\right|=\kappa_{1} .
$$

But

$$
V[G]|| \mathcal{P}\left(\kappa_{0}\right)|\leqslant|{ }^{\kappa_{0}} \mathscr{G}_{\mathbf{P}\left(\kappa_{0}\right) * \mathbf{R}\left(\kappa_{0}, \kappa_{1}\right)} \cap V \mid
$$

and

$$
\left|{ }^{\kappa_{0}} g_{\mathbf{P}\left(\kappa_{0}\right) * \mathbf{R}\left(\kappa_{0}, \kappa_{1}\right)}\right|^{V}=\kappa_{1}
$$

so $V[G] \vDash\left|\mathcal{P}\left(\kappa_{0}\right)\right|=\kappa_{1}$. 
All that remains to be checked is that $V[G]=\left|2^{\aleph_{0}}\right|=\kappa_{0}$ :

Claim. In $V^{\mathbf{P}\left(\kappa_{0}\right)}, \mathbf{R}\left(\kappa_{0}, \kappa_{1}\right)$ is a $\left(\kappa_{0}, \infty\right)$-distributive partial ordering.

The claim implies that we only have to check $2^{\kappa_{0}}=\kappa_{1}$ in $V\left[G \mid \mathbf{P}\left(\kappa_{0}\right)\right]$. Since $\mathbf{P}\left(\kappa_{0}\right)$ is $\kappa_{0}$ c.c. arguments similar to those above show that $2^{\kappa_{0}}=\kappa_{1}$ in $V[G]$.

Proof OF ClaIM. Let $\left\langle D_{\alpha}: \alpha<\beta\right\rangle\left(\beta<\kappa_{0}\right)$ be a collection of dense open subsets of $\mathbf{R}\left(\kappa_{0}, \kappa_{1}\right)$. Suppose $V^{\mathbf{P}\left(\kappa_{0}\right)}$ F there is no $\tau \in \bigcap_{\alpha<\beta} D_{\alpha}$.

Let $\hat{H}$ be a $V$-generic object for $\mathbf{P}\left(\kappa_{1}\right)$. Let $H * G$ be the generic object for $\mathbf{P}\left(\kappa_{0}\right) * \mathbf{R}\left(\kappa_{0}, \kappa_{1}\right)$ induced by icon ${ }^{\left(\kappa_{0}, \kappa_{1}\right)}$.

There is an elementary embedding $j^{*}: V[H] \rightarrow M[\hat{H}]$ extending $j$.

$$
j^{*}\left(\left\langle D_{\alpha}: \alpha<\beta\right\rangle\right)=\left\langle j^{*}\left(D_{\alpha}\right): \alpha<\beta\right\rangle,
$$

since $\beta<\kappa_{0}$. By elementarity of $j^{*}, M[\hat{H}]$ k there is no $\tau$ such that for all $\alpha<\beta$, $\tau \in j\left(D_{\alpha}\right)$.

Let $\tau \in \mathbf{R}\left(\kappa_{1}, \kappa_{2}\right), \tau \in V^{\mathbf{P}\left(\kappa_{1}\right)}$ be the master condition we constructed. Then $V[\hat{H}]$ : for all $(p, s) \in H * G, \tau<j(s)$.

However, $G$ is $V[H]$ generic and hence for each $\alpha$ there is an $s \in D_{\alpha} \cap G$. But then $j(s) \in j\left(D_{\alpha}\right)$ and $\tau \leqslant j(s)$. This is a contradiction because it implies that $\tau \in j\left(D_{\alpha}\right)$ for all $\alpha$. This completes the proof that the G.C.H. holds in $L[B]$. We still must see that $L[B]=\left(\boldsymbol{\aleph}_{3}, \boldsymbol{\aleph}_{2}, \boldsymbol{\aleph}_{1}\right) \rightarrow\left(\boldsymbol{\aleph}_{2}, \boldsymbol{\aleph}_{1}, \boldsymbol{\aleph}_{0}\right)$.

Let $\mathfrak{A} \in L[B]$ be of type $\left(\aleph_{3}, \aleph_{2}, \aleph_{1}\right)$. Then in $V[G]$ there is a $\mathfrak{B} \prec \mathfrak{A}$ of type $\left(\aleph_{2}, \aleph_{1}, \aleph_{0}\right) . B$ can be coded as a subset of $\kappa_{1}$ and hence $\mathfrak{B} \in L[B]$. Since the notion of being an elementary substructure is absolute, $L[B]=\left(\boldsymbol{\aleph}_{3}, \boldsymbol{\aleph}_{2}, \boldsymbol{\aleph}_{1}\right) \rightarrow\left(\boldsymbol{\aleph}_{2}, \boldsymbol{\aleph}_{1}, \boldsymbol{\aleph}_{0}\right)$. This completes the lemma.

To find a $\mathbf{P}$ for getting $\left(\boldsymbol{\aleph}_{n+3}, \boldsymbol{\aleph}_{n+2}, \boldsymbol{\aleph}_{n+1}\right) \rightarrow\left(\boldsymbol{\aleph}_{n+2}, \boldsymbol{\aleph}_{n+1}, \boldsymbol{\aleph}_{n}\right)$, we do our old construction taking steps to make $\mathbf{P}\left(\kappa_{0}\right)<\boldsymbol{\aleph}_{n}$-closed. We need the following lemma.

LEMMA 8.2. Suppose $\lambda$ is a regular cardinal.

(a) Let $e: \mathbf{Q} \rightarrow \mathbf{P}$. Suppose $\mathbf{P}$ is $<\lambda$-closed and $V^{\mathbf{Q}}=S$ is $<\lambda$-closed.

Then $\mathbf{P} *_{e} S$ is $<\lambda$ closed.

(b) Let $\mathbf{P}=\mathbf{P}_{\tau}$ be an iteration with support $\mathscr{K}$ such that

(i) $X \subseteq \mathcal{K},|X|<\lambda \Rightarrow U X \in \mathcal{K}$.

(ii) If $e_{\alpha}: \mathbf{Q}_{\alpha} \rightarrow \mathbf{P}_{\alpha}, \mathbf{P}_{\alpha+1}=\mathbf{P}_{\alpha} *_{e_{\alpha}} S_{\alpha}$ then $V^{\mathbf{Q}_{\alpha}} S_{\alpha}$ is $<\lambda$ closed.

Then $\mathbf{P}$ is $<\lambda$ closed.

We leave the proof of this lemma to the reader.

From the lemma, we see that if $\mathbf{Q}$ is $<\lambda$ closed and $\kappa$ c.c. with $\lambda<\kappa$, then $\mathbf{Q} * \mathbf{R}(\kappa, \mu, X,<)$ is $<\lambda$ closed. Suppose we want to show $\left(\boldsymbol{\aleph}_{n+3}, \boldsymbol{\aleph}_{n+2}, \boldsymbol{\aleph}_{n+1}\right) \rightarrow\left(\boldsymbol{\aleph}_{n+2}, \boldsymbol{\aleph}_{n+1}, \boldsymbol{\aleph}_{n}\right)$. Then as before we build a partial order $\mathbf{P}^{\prime}$ and a generic elementary embedding $\hat{j}: V^{\mathbf{P}^{\prime}} \rightarrow M^{j\left(\mathbf{P}^{\prime}\right)}$ extending $j$, such that in $V^{\mathbf{P}^{\prime}}$, $\kappa_{0}=\boldsymbol{\aleph}_{n+1}, \kappa_{1}=\boldsymbol{\aleph}_{n+2}, \kappa_{2}=\boldsymbol{\aleph}_{n+3}$. To find such a $\mathbf{P}^{\prime}$ we do things exactly as before except that for any $\alpha$ Mahlo we let $\mathbf{P}_{0}^{\prime}(\alpha)=S\left(\boldsymbol{\aleph}_{n}, \alpha\right)$. (Here $\mathbf{P}^{\prime}(\alpha)$ takes the place of the old $\mathbf{P}(\alpha)$.) Such a $\mathbf{P}^{\prime}(\alpha)$ satisfies the hypothesis for Lemma 8.2.

As a further result down these lines, the author has shown: Con(ZFC + there is a 2-huge cardinal) $\Rightarrow \operatorname{Con}(\mathrm{ZFC}+$ for all $m, n \in \omega, m>n$ implies $\left.\left(\aleph_{m+1}, \aleph_{m}\right) \rightarrow\left(\aleph_{n+1}, \aleph_{n}\right)\right)$. This will appear in a later paper. 
ACKNOWLEDGEMENT. The contents of this paper are essentially the author's Ph.D. Thesis at the University of California, Berkeley. The author would like to thank his advisor, Professor Robert Solovay, for his patience and constant helpful comments. Thanks should also go to Professor Richard Laver, who suggested the problem, and to Charles Gray for helpful conversations about Set Theory in general. Finally, the author would like to thank Professor Jerome Malitz without whose encouragement the thesis would not have been possible.

\section{BIBLIOGRAPHY}

1. J. Baumgartner, Iterated forcing, unpublished notes.

2. K. Kunen, Saturated ideals, J. Symbolic Logic 43 (1978), 65-76.

3. R. Laver, private communications.

4. J. Silver, private communications.

5. R. M. Solovay, W. N. Reinhardt and A. Kanamori, Strong axioms of infinity and elementary embeddings, Ann. Math. Logic 13 (1978), 73-116.

Department of Mathematics, University of California at los Angeles, Los Angeles, CaliFORNIA 90024 